\title{
Cauchy Problems for Mixed-Type Operators
}

\author{
By
}

\author{
Keisuke UchIKOSHI*
}

\begin{abstract}
We give a general theory of the Cauchy problems for various types of operators, containing hyperbolic operators, elliptic operators, and mixed-type operators. We will give a necessary and sufficient condition for the Cauchy problems to be well-posed.
\end{abstract}

\section{\$1. Introduction}

The aim of this paper is to give a general theory for Cauchy problems, applicable for hyperbolic operators, elliptic operators, and mixed-type operators. For example, it will turn out that we can consider Cauchy problems for

$$
\left.P=\left(D_{1}^{2}-x_{1} D_{n}^{2}\right)\left(D_{1}^{2}-x_{1}^{2} D_{n}^{2}\right)\left(D_{1}^{2}+x_{1}^{2} D_{n}^{2}\right)+\text { (fifth-order operator }\right)
$$

The general theory is as follows. Let $P(x, D)$ be a microdifferential operator defined at $x^{*}=(0 ; 0, \ldots, 0, \sqrt{-1}) \in \sqrt{-1} T^{*} \mathbf{R}^{n}$ of order $m \geq 2$, written in the form

$$
P(x, D)=D_{1}^{m}+\sum_{0 \leq j \leq m-1} P_{j}\left(x, D^{\prime}\right) D_{1}^{j}, \quad \text { ord } P_{j} \leq m-j .
$$

Here we have written $D=\partial / \partial x$, and $D^{\prime}=\left(D_{2}, \ldots, D_{n}\right)$. We also write as $D^{\prime \prime}=\left(D_{1}, \ldots, D_{n-1}\right), D^{\prime \prime \prime}=\left(D_{2}, \ldots, D_{n-1}\right)$. Let $\sigma_{m}(P)(x, \xi)$ be the principal symbol of $P(x, D)$. We assume that

$$
\left\{\begin{array}{l}
\text { if } x_{1}=0, \text { then } \sigma_{m}(P)=\xi_{1}^{m} \\
\text { if } x_{1} \neq 0, \text { then the equation } \sigma_{m}(P)=0 \text { has } m \text { distinctive roots } \\
\xi_{1}=\varphi_{1}\left(x, \xi^{\prime}\right), \ldots, \varphi_{m}\left(x, \xi^{\prime}\right) .
\end{array}\right.
$$

Communicated by T. Kawai, March 15, 1999. Revised December 1, 1999.

1991 Mathematics subject classification: Primary 35M10, Secondly 35J70, 35L80.

* Department of Mathematics, National Defense Academy, Hashirimizu 1-10-20, Yokosuka, Japan.

e-mail: uchikosh@cc.nda.ac.jp 
We denote by $\mathcal{O}$ the sheaf of holomorphic functions, and we define $\mathcal{O}_{(j)}=$ $\sum_{0 \leq k \leq j-1} x_{1}^{k / j} \mathcal{O}$ for $j \in \mathbf{N}$. Without loss of generality, we may assume that for $\exists q_{j} \in \mathbf{N} / m^{\prime}, \exists a_{j}\left(x, \xi^{\prime}\right) \in \mathcal{O}_{\left(m^{\prime}\right), x^{*}}$ we have $\varphi_{j}\left(x, \xi^{\prime}\right)=x_{1}^{q_{J}} a_{j}\left(x, \xi^{\prime}\right), a_{j}\left(x^{*}\right) \neq 0$ $(1 \leq j \leq m)$. Here each $\varphi_{j}\left(x, \xi^{\prime}\right)$ is homogeneous in $\xi^{\prime}$ of degree 1 . We also assume that

$$
i \neq j \Rightarrow\left(q_{i}, a_{i}\left(x^{*}\right)\right) \neq\left(q_{j}, a_{j}\left(x^{*}\right)\right) .
$$

We denote by $\mathscr{C}$ (resp. $\mathscr{E}$ ) the sheaf of microfunctions (resp. microdifferential operators). Let us consider the Cauchy problem

$$
\left\{\begin{array}{l}
P u=0, \\
D_{1}^{j-1} u\left(0, x^{\prime}\right)=v_{j}\left(x^{\prime}\right), \quad 1 \leq j \leq m,
\end{array}\right.
$$

where $u \in \mathscr{C}_{\mathbf{R}^{n}, x^{*}}$ and $v_{j} \in \mathscr{C}_{\mathbf{R}^{n-1}, x^{* \prime}}\left(x^{* \prime}=(0 ; 0, \ldots, 0, \sqrt{-1}) \in \sqrt{-1} T^{*} \mathbb{R}^{n-1}\right)$. If $P(x, D)$ is microhyperbolic, (5) is well-posed for arbitrary initial values, as is well-known (See [5]). Otherwise (5) may be solvable for some initial values (e.g., for $v_{1}=\cdots=v_{m}=0$ ), but may be unsolvable for other initial values. Therefore there arises a problem to know for which initial values (5) becomes solvable.

To give the main theorem we need to give some preliminaries. Let $A\left(x^{\prime}, D^{\prime}\right)$ be a both-side invertible $m \times m$ matrix whose components $A_{(\mu, v)}\left(x^{\prime}, D^{\prime}\right) \in \mathscr{E}_{x^{*}}^{\mathbb{R}}$ are independent of $\left(x_{1}, D_{1}\right)$. Here we denote by $\mathscr{E} \mathbf{R}$ the sheaf of holomorphic microlocal operators (c.f. [2,9]). We choose $r$ rows of this matrix in an arbitrary way. To be clear, let $1 \leq j_{1}<j_{2}<\cdots<j_{r} \leq m$ and choose the $j_{1}, \ldots, j_{r}$-th rows of $A$. Then we obtain an $r \times m$ matrix $A^{\prime}\left(x^{\prime}, D^{\prime}\right)$ of holomorphic microlocal operators. Let us choose some $r$ rows of some $A$. We say that $v_{1}\left(x^{\prime}\right), \ldots, v_{m}\left(x^{\prime}\right) \in \mathscr{C}_{\mathbf{R}^{n-1}, x^{*}}$ satisfy an $r$-relation (defined by $A^{\prime}$ ) if $A^{\prime}\left(x^{\prime}, D^{\prime}\right) \vec{v}\left(x^{\prime}\right)=\overrightarrow{0}$. Here $\vec{v}$ denotes ${ }^{t}\left(v_{1}, \ldots, v_{m}\right)$, and we denote the set of such vectors by $\left(\mathscr{C}_{\mathbf{R}^{n-1}, x^{*}}\right)^{m}$. We have $A:\left(\mathscr{C}_{\mathbf{R}^{n-1}, x^{* \prime}}\right)^{m} \stackrel{\sim}{\rightarrow}\left(\mathscr{C}_{\mathbf{R}^{n-1}, x^{*}}\right)^{m}$, and an $r$ relation means that $r$ of the components of $\vec{v}$ disappear when it is sent to righthand side. We note that even if $v_{1}\left(x^{\prime}\right), \ldots, v_{m}\left(x^{\prime}\right)$ satisfy an $r$-relation and another $s$-relation, it does not necessarily mean an $(r+s)$-relation.

We next define a classification of the characteristic roots. Let $\theta \in\{0, \pi\}$. Let $\omega \subset \mathbb{R}^{n} \times \sqrt{-1} \mathbb{R}^{n-1}$ be a small neighborhood of $x^{*}$, and let $\omega_{\theta}=$ $\left\{\left(x, \xi^{\prime}\right) \in \omega ; x_{1} \neq 0, \arg x_{1}=\theta\right\}$. We define

$$
\begin{aligned}
M & =\{1,2, \ldots, m\}, \\
M_{0, \theta} & =\left\{\lambda \in M ; \operatorname{Re}\left(x_{1} \varphi_{\lambda}\left(x, \xi^{\prime}\right)\right)=0, \text { if }\left(x, \xi^{\prime}\right) \in \omega_{\theta}\right\}, \\
M_{ \pm, \theta} & =\left\{\lambda \in M ; \pm \operatorname{Re}\left(x_{1} \varphi_{\lambda}\left(x, \xi^{\prime}\right)\right)>0, \text { if }\left(x, \xi^{\prime}\right) \in \omega_{\theta}\right\}, \\
M_{\theta}^{\prime} & =M \backslash M_{0, \theta} \backslash M_{+, \theta} \backslash M_{-, \theta} .
\end{aligned}
$$


To give another expression of this definition, we consider the solutions $\psi^{(+, \lambda)}\left(x, \xi^{\prime}\right)$ of the following phase equation:

$$
\left\{\begin{array}{l}
\partial_{x_{1}} \psi^{(+, \lambda)}-\varphi_{\lambda}\left(x, \xi^{\prime}+\partial_{x^{\prime}} \psi^{(+, \lambda)}\right)=0 \\
\psi^{(+, \lambda)}\left(0, x^{\prime}, \xi^{\prime}\right)=0
\end{array}\right.
$$

and define $\psi^{(-, \lambda)}\left(x, \xi^{\prime}\right)$ by

$$
\left\{\begin{array}{l}
\partial_{x_{1}} \psi^{(-, \lambda)}+\varphi_{\lambda}\left(x_{1}, x^{\prime}+\partial_{\xi^{\prime}} \psi^{(\lambda)}, \xi^{\prime}\right)=0 \\
\psi^{(-, \lambda)}\left(0, x^{\prime}, \xi^{\prime}\right)=0
\end{array}\right.
$$

Then it is easy to see that

$$
\begin{aligned}
M_{0, \theta} & =\left\{\lambda \in M ; \operatorname{Re} \psi^{(+, \lambda)}\left(x, \xi^{\prime}\right)=0\right\}, \\
& =\left\{\lambda \in M ; \operatorname{Re} \psi^{(-, \lambda)}\left(x, \xi^{\prime}\right)=0\right\}, \\
M_{ \pm, \theta} & =\left\{\lambda \in M ; \pm \operatorname{Re} \psi^{(+, \lambda)}\left(x, \xi^{\prime}\right)>0\right\}, \\
& =\left\{\lambda \in M ; \pm \operatorname{Re} \psi^{(-, \lambda)}\left(x, \xi^{\prime}\right)<0\right\} .
\end{aligned}
$$

Let $m_{0, \theta}, m_{ \pm, \theta}$ be the numbers of the elements belonging to $M_{0, \theta}, M_{ \pm . \theta}$, respectively. We always assume that

$$
M_{0}^{\prime}=M_{\pi}^{\prime}=\varnothing
$$

Example. If we have

$$
\left\{\begin{array}{l}
\varphi_{1}\left(x, \xi^{\prime}\right)=x_{1} \xi_{n} \\
\varphi_{2}\left(x, \xi^{\prime}\right)=\sqrt{-1} x_{1} \xi_{n} \\
\varphi_{3}\left(x, \xi^{\prime}\right)=-\sqrt{-1} x_{1} \xi_{n} \\
\varphi_{4}\left(x, \xi^{\prime}\right)=x_{1}^{1 / 2} \xi_{n} \\
\varphi_{5}\left(x, \xi^{\prime}\right)=-x_{1}^{1 / 2} \xi_{n} \\
\varphi_{6}\left(x, \xi^{\prime}\right)=x_{1}^{2}\left(1+\sqrt{-1} x_{2}\right) \xi_{n}
\end{array}\right.
$$

then the above classification is as follows. We have $\left(x, \xi^{\prime}\right) \in \mathbf{R}^{n} \times \sqrt{-1} \mathbf{R}^{n-1}$, $\operatorname{Im} \xi_{n}>0$, and $\arg x_{1}=\theta, \arg \xi_{n}=\pi / 2$. Therefore we have $\operatorname{Re}\left(x_{1} \varphi_{1}\left(x, \xi^{\prime}\right)\right)$ $=0$, and $1 \in M_{0, \theta}$. Similarly we have $2 \in M_{-, \theta}, 3 \in M_{+, \theta}$ (for each $\theta \in\{0, \pi\}$ ). We next note that $\arg \left(x_{1} \varphi_{4}\left(x, \xi^{\prime}\right)\right)=3 \theta / 2+\pi / 2$. It follows that $4 \in M_{0,0}$, $4 \in M_{+, \pi}$. Similarly we have $5 \in M_{0,0}, 5 \in M_{-, \pi}$. Finally we have

$$
\operatorname{Re}\left(x_{1} \varphi_{6}\left(x, \xi^{\prime}\right)\right)=\operatorname{Re}\left(x_{1}^{3}\left(1+\sqrt{-1} x_{2}\right) \xi_{n}\right)=-\operatorname{Re}\left(x_{1}^{3} x_{2}\right) \operatorname{Im} \xi_{n}
$$

and it follows that $6 \in M_{\theta}^{\prime}$. This means that the types of $\varphi_{4}\left(x, \xi^{\prime}\right), \varphi_{5}\left(x, \xi^{\prime}\right)$ change from elliptic to hyperbolic as $x_{1}$ varies, but that of $\varphi_{6}\left(x, \xi^{\prime}\right)$ changes as $x_{1}, x_{2}$ vary. Our assumption (7) means that we do not consider such a 
complicated characteristic root as $\varphi_{6}\left(x, \xi^{\prime}\right)$. Let us summarize the above calculation.

\begin{tabular}{l|llll}
\hline$\theta$ & $M_{0, \theta}$ & $M_{+, \theta}$ & $M_{-, \theta}$ & $M_{\theta}^{\prime}$ \\
\hline 0 & $\{1,4,5\}$ & $\{3\}$ & $\{2\}$ & $\{6\}$ \\
$\pi$ & $\{1\}$ & $\{3,4\}$ & $\{2,5\}$ & $\{6\}$ \\
\hline
\end{tabular}

Now we give the main result.

Theorem 1. We assume (2), (3), (4), and (7). Then there exist an $m_{+, 0^{-}}$ relation and an $m_{+, \pi}$-relation (which are defined only by $P$ ), such that the Cauchy problem (5) has a solution $u \in \mathscr{C}_{\mathbf{R}^{n}, x^{*}}$ if, and only if, $v_{1}\left(x^{\prime}\right), \ldots, v_{m}\left(x^{\prime}\right) \in \mathscr{C}_{\mathbf{R}^{n-1}, x^{* \prime}}$ satisfy both these relations.

We give some examples. At first we remind the reader of the well-known result for the operators of principal type.

Example (Lewy-Mizohata operators). If $P_{ \pm}=D_{1} \pm \sqrt{-1} x_{1} D_{n}$, then we have $M_{ \pm, \theta}=\{1\}(=M), M_{\mp, \theta}=\varnothing$. This means that $P_{-} u=0, u\left(0, x^{\prime}\right)=$ $v\left(x^{\prime}\right)$ is solvable for any $v \in \mathscr{C}_{\mathbf{R}^{n-1}, x^{* \prime}}$ without any relations, which coincides with the well-known result. On the other hand, $P_{+} u=0, u\left(0, x^{\prime}\right)=v\left(x^{\prime}\right)$ is solvable only for the case when $v\left(x^{\prime}\right)$ satisfies a one-relation. This means $v=0$, and $u=0$. It follows that $P_{+} u=0 \Rightarrow u=0$, i.e., $P_{+}$is hypo-elliptic (See [9]).

Lewy-Mizohata operators are the simplest case of our theory, and our theorem gives a similar result even for more complicated operators. The characteristic roots belonging to $M_{+, \theta}$ cause obstruction, and correspondingly the Cauchy data must satisfy suitable relations. Let us see the case $m=2$.

Example (microhyperbolic operators). Let $P(x, D)=D_{1}^{2}-x_{1}^{2} D_{n}^{2}+$ $P^{\prime}(x, D)$, ord $P^{\prime} \leq 1$ (Without loss of generality, we may assume that $P^{\prime}$ is a polynomial in $D_{1}$ of degree 1). In this case we have $\varphi_{1}\left(x, \xi^{\prime}\right)=x_{1} \xi_{n}, \varphi_{2}\left(x, \xi^{\prime}\right)$ $=-x_{1} \xi_{n}$. It follows that $M_{0, \theta}=\{1,2\}, M_{ \pm, \theta}=\varnothing$ for $\theta \in\{0, \pi\}$. This means that (5) is solvable for an arbitrary $v_{1}\left(x^{\prime}\right), v_{2}\left(x^{\prime}\right) \in \mathscr{C}_{\mathbf{R}^{n-1}, x^{* \prime}}$ without any relations (See $[1,7,8,10,13])$.

Example (Tricomi operators). Let $P(x, D)=D_{1}^{2}-x_{1} D_{n}^{2}+P^{\prime}(x, D)$, ord $P^{\prime} \leq 1$. We have $\varphi_{1}\left(x, \xi^{\prime}\right)=\sqrt{x_{1}} \xi_{n}, \varphi_{2}\left(x, \xi^{\prime}\right)=-\sqrt{x_{1}} \xi_{n}$. It follows that $M_{0,0}=\{1,2\}, \quad m_{+, 0}=0, \quad$ and that $M_{+, \pi}=\{1\}, \quad M_{-, \pi}=\{2\}, \quad m_{+, \pi}=1$. Therefore there exists a 1-relation, and (5) is solvable if, and only if, the Cauchy data satisfy this relation. This case was investigated by [7].

Example (hypoelliptic operators). Let $P(x, D)=D_{1}^{2}+x_{1}^{2} D_{n}^{2}+P^{\prime}(x, D)$, ord $P^{\prime} \leq 1$. Since $\varphi_{1}\left(x, \xi^{\prime}\right)=\sqrt{-1} x_{1} \xi_{n}, \varphi_{2}\left(x, \xi^{\prime}\right)=-\sqrt{-1} x_{1} \xi_{n}$, it is easy to see 
that $M_{-, \theta}=\{1\}, M_{+, \theta}=\{2\}, m_{+, \theta}=1$ for $\theta \in\{0, \pi\}$. It follows that (5) is solvable if, and only if, $v_{1}, v_{2}$ satisfy a 1-relation and another 1-relation. If these two 1-relations mean a 2-relation, (5) is solvable only in the case $v_{1}=$ $v_{2}=0$, and we have $u=0$. In other words, $P u=0$ does not have non-trivial solutions. It is well-known that this is true if the principal symbol $\sigma_{1}\left(P^{\prime}\right)$ of the lower order term satisfies $\xi_{n}^{-1} \sigma_{1}\left(P^{\prime}\right) \notin\{\sqrt{-1}, 3 \sqrt{-1}, 5 \sqrt{-1}, \ldots\}$ (See [4]).

Of course our result applies for higher order operators. For instance, let us consider the Cauchy problem (5) for the sixth-order operator (1). Then the Cauchy data $v_{1}, \ldots, v_{6}$ must satisfy a 1-relation and a 2-relation. Roughly speaking, we can give three microfunctions arbitrarily among $v_{1}, \ldots, v_{6}$.

In order to prove Theorem 1 , we shall show that fixing $\arg x_{1}$, we can give a canonical representation of the elementary solution of $P(x, D)$. This result has its own interest. But to give its precise statement, we need to prepare a symbol theory. Therefore in the next section we shall give such a symbol theory, and we postpone the discussion of the canonical form until Section 3.

\section{§2. An Operator Theory}

In this section we give an operator theory, which is necessary for the proof of Theorem 1. We may assume that $q_{1} \leq \cdots \leq q_{m}$, and we define $q=$ $\max \left(q_{1}, \ldots, q_{m}\right)\left(=q_{m}\right)$.

\section{§2.1. A Theory of Formal Operators}

Let $C>0, i \in \mathbf{Z}_{+}=\{0,1,2, \ldots\}$. We define

$$
\begin{aligned}
& \Omega(C)=\left\{\left(x, \xi^{\prime}\right) \in \mathbf{C}^{n} \times \mathbf{C}^{n-1} ; C|x|<1,\right. \\
&\left.C\left|\xi^{\prime \prime \prime}\right|<\operatorname{Im} \xi_{n}, C\left|\operatorname{Re} \xi_{n}\right|<\operatorname{Im} \xi_{n}, C^{4 m q}<\operatorname{Im} \xi_{n}\right\}, \\
& \Omega_{i}(C)=\left\{\left(x, \xi^{\prime}\right) \in \Omega(C) ; C^{4 m q}(i+1)<\operatorname{Im} \xi_{n}\right\} .
\end{aligned}
$$

Assume that for $\exists C>0, \exists R \in(0,1), \forall \varepsilon>0, \exists C_{\varepsilon}>0$ a formal series $f=$ $\sum_{i \in \mathbf{Z}_{+}} f_{i}\left(x, \xi^{\prime}\right)$ satisfies $f_{i} \in \mathcal{O}\left(\Omega_{i}(C)\right)$ and

$$
\left|f_{i}\left(x, \xi^{\prime}\right)\right| \leq C_{\varepsilon} R^{i} \exp \left(\left(C\left|x_{1}\right|^{1+\left(1 / m^{\prime}\right)}+C\left(\left|\operatorname{Re} \xi^{\prime}\right| / \operatorname{Im} \xi_{n}\right)^{2}+\varepsilon\right) \operatorname{Im} \xi_{n}\right)
$$

on $\Omega_{i}(C)$. We denote by $\mathscr{T}(\Omega(C))$ the set of such formal series. If $f=\sum_{i} f_{i}$, we define a formal series $f^{\#}=\sum_{i} f_{i}^{\#}$ by $f_{i}^{\#}=\sum_{0 \leq j \leq i} f_{j}$, and $\mathcal{N}(\Omega(C))$ by

$$
\mathscr{N}(\Omega(C))=\left\{f \in \mathscr{T}(\Omega(C)) ; f^{\#} \in \mathscr{T}(\Omega(C))\right\} .
$$


Finally we define

$$
\mathscr{T}=\underset{C>0}{\lim _{\longrightarrow}} \mathscr{T}(\Omega(C)), \quad \mathscr{N}=\underset{C>0}{\lim } \mathscr{N}(\Omega(C)) .
$$

Remark. (i) According to the useful method of Aoki [2], we can neglect the elements of $\mathscr{N}$, as we shall see.

(ii) We write $\sum f_{i}=\sum g_{i}$ if, and only if, $f_{i}=g_{i}$ for any $i$. Since this does not merely mean that the sums of these two series are the same, we sometimes write as $\sum f_{i}=\sum g_{i}$.

(iii) We identify a function $f_{0}$ with a formal series $f_{0}+0+0+\cdots \epsilon$ $\mathscr{T}(\Omega(C))$, if it satisfies (8) for $i=0$. A function $f_{0}$ belongs to $\mathcal{N}(\Omega(C))$ if, and only if, it is exponentially decreasing.

(iv) For a formal series $f=\sum f_{i}$ we define $\partial_{x_{1}} f=\sum \partial_{x_{1}} f_{i}$.

Let $f=\sum f_{i} \in \mathscr{T}(\Omega(C))$. We next define

$$
\mathscr{F}(f)\left(x, y^{\prime}\right)=(2 \pi \sqrt{-1})^{-n+1} \sum_{i} \int e^{\xi^{\prime} \cdot\left(x^{\prime}-y^{\prime}\right)} f_{i}\left(x, \xi^{\prime}\right) d \xi^{\prime},
$$

and show that it defines a formal operator. To be precise, we use an analytic partition of the unity. Let $C^{\prime} \gg C$. We consider the following linear transformation:

$$
\begin{cases}\tilde{\xi}_{j}=-C^{\prime} \xi_{j}+\xi_{n}, & 2 \leq j \leq n-1, \\ \tilde{\xi}_{j}=C^{\prime}\left(\xi_{2}+\cdots+\xi_{n-1}\right)+\xi_{n}, & j=n .\end{cases}
$$

Then $\sqrt{-1} \mathbb{R}^{n-1} \in \xi^{\prime} \mapsto \tilde{\xi}^{\prime} \in \sqrt{-1} \mathbb{R}^{n-1}$ is an isomorphism, and the first octant $A=\left\{\tilde{\xi}^{\prime} \in \mathbb{C}^{n-1} ; \operatorname{Im} \tilde{\xi}_{j}>C^{\prime}\left|\operatorname{Re} \tilde{\xi}_{j}\right|, 2 \leq j \leq n\right\}$ corresponds to a small neighborhood of $\xi^{\prime}=(0, \ldots, 0, \sqrt{-1}) \in \mathbb{C}^{n-1}$. We define the central region $A^{\text {ce }}$ and the boundary region $A^{\text {bo }}$ of $A$ by $A^{\text {ce }}=\left\{\tilde{\xi}^{\prime} \in A ; n^{2} \operatorname{Im} \tilde{\xi}_{j}>\operatorname{Im} \tilde{\xi}_{k}, \forall j, \forall k\right\}$ and $A^{\text {bo }}=\left\{\tilde{\xi}^{\prime} \in A ; C^{\prime} \operatorname{Im} \tilde{\xi}_{j}<\operatorname{Im} \tilde{\xi}_{k}, \exists j, \exists k\right\}$, respectively. There exists $e\left(\tilde{\xi}^{\prime}\right) \in \mathcal{O}(A)$ such that for $\forall \varepsilon>0, \exists C_{\varepsilon}>0$

$$
\begin{gathered}
\left|e\left(\tilde{\xi}^{\prime}\right)\right| \leq C_{\varepsilon} \exp \left(\left(C^{\prime}\left|\operatorname{Re} \tilde{\xi}^{\prime}\right|^{2}\left(\operatorname{Im} \tilde{\xi}_{n}\right)^{-2}+\varepsilon\right) \operatorname{Im} \tilde{\xi}_{n}\right) \quad \text { on } A \\
\left|e\left(\tilde{\xi}^{\prime}\right)-1\right| \leq C^{\prime} \exp \left(-C^{\prime-1} \operatorname{Im} \tilde{\xi}_{n}\right) \quad \text { on } A^{\text {ce }} \\
\left|e\left(\tilde{\xi}^{\prime}\right)\right| \leq C^{\prime} \exp \left(-C^{\prime-1} \operatorname{Im} \tilde{\xi}_{n}\right) \quad \text { on } A^{\text {bo }}
\end{gathered}
$$

(See [11]).

Let $f=\sum f_{i}\left(x, \xi^{\prime}\right) \in \mathscr{T}(\Omega(C))$. From (9) it follows that $e f=\sum e\left(\tilde{\xi}^{\prime}\right)$. $f_{i}\left(x, \xi^{\prime}\right) \in \mathscr{T}\left(\Omega\left(C^{\prime \prime}\right)\right)$ for some $C^{\prime \prime}$. From $(10)$ we have $e\left(\tilde{\xi}^{\prime}\right)=1$, ef $=f$ in $\mathscr{T} / \mathcal{N}$.

We define $\mathscr{F}(f)\left(x, y^{\prime}\right)$ by 


$$
\mathscr{F}(f)\left(x, y^{\prime}\right)=(2 \pi \sqrt{-1})^{-n+1} \sum_{i} \int_{\Delta_{l}\left(C^{\prime \prime}\right)} e^{\xi^{\prime} \cdot\left(x^{\prime}-y^{\prime}\right)} e\left(\xi^{\prime}\right) f_{i}\left(x, \xi^{\prime}\right) d \xi^{\prime},
$$

where $\Delta_{i}\left(C^{\prime \prime}\right)=\left\{\xi^{\prime} \in \sqrt{-1} \mathbb{R}^{n-1} ; \tilde{\xi}_{j}>C^{\prime \prime}(j+1), 2 \leq j \leq n\right\}$.

We define $\zeta^{\prime o}=(0, \ldots, 0,1) \in \mathbf{R}^{n-1}, Z^{\prime}=\left\{\zeta^{\prime} \in \mathbf{R}^{n-1} ;\left|\zeta^{\prime}\right|=1,-1<\zeta_{n}<0\right\}$ and

$$
\begin{aligned}
W(C, r)=\{ & \left(x, y^{\prime}\right) \in \mathbb{C}^{n} \times \mathbf{C}^{n-1} ; C\left|\left(x, y^{\prime}\right)\right|<1, \\
& \left.\operatorname{Im}\left(x_{n}-y_{n}\right)+C^{-1}\left|\operatorname{Re}\left(x^{\prime}-y^{\prime}\right)\right|^{2}>r\left|\operatorname{Im}\left(x^{\prime \prime \prime}-y^{\prime \prime \prime}\right)\right|+C\left|x_{1}\right|^{1+\left(1 / m^{\prime}\right)}\right\}, \\
W\left(C, \delta, \zeta^{\prime}\right)= & \left\{\left(x, y^{\prime}\right) \in \mathbf{C}^{n} \times \mathbf{C}^{n-1} ; C\left|\left(x, y^{\prime}\right)\right|<1,\left.\operatorname{Im}\left(x^{\prime}-y^{\prime}\right)|>C| x_{1}\right|^{1+\left(1 / m^{\prime}\right)},\right. \\
& \left|\frac{1}{\left|\operatorname{Im}\left(x^{\prime}-y^{\prime}\right)\right|} \operatorname{Im}\left(x^{\prime}-y^{\prime}\right)-\frac{1}{\left|\theta \zeta^{\prime o}+(1-\theta) \zeta^{\prime}\right|}\left(\theta \zeta^{\prime o}+(1-\theta) \zeta^{\prime}\right)\right| \\
& <\delta, 0 \leq \exists \theta \leq 1\}
\end{aligned}
$$

for $C, r, \delta>0, \zeta^{\prime} \in Z^{\prime}$. Then we have the following

Lemma 1. (i) Let $f=\sum f_{i}\left(x, \xi^{\prime}\right) \in \mathscr{T}(\Omega(C))$. $\mathscr{F}(f)$ is holomorphic on $W\left(C^{\prime \prime \prime}, r\right)$ for $\exists C^{\prime \prime \prime}>0$ and $\exists r>0$.

(ii) Let $C^{\prime \prime}<\tilde{C}^{\prime \prime}$. If we replace $\Delta_{i}\left(C^{\prime \prime}\right)$ by $\Delta_{i}\left(\tilde{C}^{\prime \prime}\right)$ in $(11)$, we obtain a different $\mathscr{F}(f)$. Let $\tilde{\mathscr{F}}(f)$ be the function thus obtained. Then we have $\tilde{\mathscr{F}}(f)-$ $\mathscr{F}(f)=\sum_{j: \text { finite }} \exists F_{j}\left(x, y^{\prime}\right)$, where $F_{j}\left(x, y^{\prime}\right) \in \mathcal{O}\left(W\left(C^{\prime \prime \prime}, \delta, \zeta^{\prime(j)}\right)\right)$ with some $C^{\prime \prime \prime}>0$, $\delta>0, \zeta^{\prime(j)} \in Z^{\prime}$ for each $j$.

(iii) If $f \in \mathscr{N}(\Omega(C))$, then $\mathscr{F}(f)$ is holomorphic in a neighborhood of the origin.

We can prove this lemma by an elementary calculation. Now let $\mathscr{W}=$ $\underset{C, r}{\lim } \mathcal{O}(W(C, r))$, let $\mathscr{W}_{1}$ be the set holomorphic functions defined on $W(C, r) \cup$ $W\left(C, \delta, \zeta^{\prime}\right)$ with some $C, r, \delta, \zeta^{\prime}$, and let $\mathscr{W}_{2}$ be the set of finite sums of the elements of $\mathscr{W}_{1}$. We have defined a map $\mathscr{F}: \mathscr{T} / \mathcal{N} \rightarrow \mathscr{W} / \mathscr{W}_{2}$. Similarly to [11], we can prove that this is an isomorphism. We next define a ring structure of $\mathscr{W} / \mathscr{W}_{2}$. Let $u_{1}\left(x, y^{\prime}\right), u_{2}\left(x, y^{\prime}\right) \in \mathcal{O}(W(C, r))$. Let $C^{\prime} \gg C$ and let

$$
\begin{aligned}
\Lambda\left(C^{\prime}, \varepsilon\right)=\left\{z^{\prime} \in \mathbf{R}^{n-2} \times \mathbf{C} ;\left|\operatorname{Re} z_{k}\right| \leq 2 C^{\prime-1}(2 \leq k \leq n),\right. \\
\left.\operatorname{Im} z_{n}=\max \left(-C^{\prime-3},\left(-C^{\prime-1}\left|\operatorname{Re} z^{\prime}\right|^{2}+C^{\prime}\left|x_{1}\right|^{1+\left(1 / m^{\prime}\right)}+\varepsilon\right)_{+}\right)\right\}
\end{aligned}
$$

where $t_{+}=\max (0, t)$. Then we have the following

Lemma 2. (i) Let $0<C \ll C^{\prime} \ll C^{\prime \prime}, 0<\varepsilon<C^{\prime \prime-1}$. If $\left(x, z^{\prime}\right) \in W\left(C^{\prime \prime}, r\right)$ and $x^{\prime}-y^{\prime} \in \Lambda\left(C^{\prime}, \varepsilon\right)$, then we have $\left(x, y^{\prime}\right),\left(x_{1}, y^{\prime}, z^{\prime}\right) \in W(C, r)$. 
(ii) If $u_{1}\left(x, y^{\prime}\right), u_{2}\left(x, y^{\prime}\right) \in \mathcal{O}(W(C, r))$, then

$$
u_{1} * u_{2}\left(x, z^{\prime}\right)=\int_{\Lambda\left(C^{\prime}, \varepsilon\right)} u_{1}\left(x, y^{\prime}\right) u_{2}\left(x_{1}, y^{\prime}, z^{\prime}\right) d\left(x^{\prime}-y^{\prime}\right) \in \mathcal{O}\left(W\left(C^{\prime \prime}, r\right)\right)
$$

is well-defined. Here $u_{1} * u_{2}$ does not depend on $\varepsilon$. Furthermore, if we replace $C^{\prime}$ by $\tilde{C}^{\prime}$, then the difference is holomorphic in a neighborhood of the origin.

(iii) If at least one of $u_{1}\left(x, y^{\prime}\right), u_{2}\left(x, y^{\prime}\right)$ belongs to $\mathcal{O}\left(W\left(C, \delta, \zeta^{\prime}\right)\right)$, then $u_{1} * u_{2}$ belongs to $\mathcal{O}\left(W\left(C, \tilde{\delta}, \tilde{\zeta}^{\prime}\right)\right)$ for some $\tilde{\delta}>0$ and $\tilde{\zeta}^{\prime} \in Z^{\prime}$.

Therefore we obtain a map $\mathscr{W} / \mathscr{W}_{2} \times \mathscr{W} / \mathscr{W}_{2} \ni\left(u_{1}, u_{2}\right) \mapsto u_{1} * u_{2} \in \mathscr{W} / \mathscr{W}_{2}$, and we can endow $\mathscr{W} / \mathscr{W}_{2}$ with a ring structure with the unit element $\mathscr{F}(1)$. Furthermore, we can easily prove the following

Lemma 3. Let $\sum f_{j}, \sum g_{j} \in \mathscr{T}(\Omega(C))$. We define $h=\sum_{j} h_{j}\left(x, \xi^{\prime}\right)$ by

$$
h_{j}=\sum_{k+\ell+\left|\alpha^{\prime}\right|=j} \frac{1}{\alpha^{\prime} !} \partial_{\xi^{\prime}}^{\alpha^{\prime}} f_{k} \partial_{x^{\prime}}^{\alpha^{\prime}} g_{\ell} .
$$

Then we have $\sum h_{j} \in \mathscr{T}\left(\Omega\left(C^{\prime}\right)\right)$ for $C^{\prime} \gg C$, and as an element of $\mathscr{W} / \mathscr{W}_{2}$ we have $\mathscr{F}(h)=\mathscr{F}(f) * \mathscr{F}(g)$. (We denote $\sum_{j} h_{j}$ also by $\left.f \circ g\right)$.

Let us define formal operands corresponding to these operators. We define

$$
\begin{aligned}
V(C, r)= & \left\{x \in \mathbb{C}^{n} ; C|x|<1, \operatorname{Im} x_{n}>r\left|\operatorname{Im} x^{\prime \prime \prime}\right|+C\left|x_{1}\right|^{1+\left(1 / m^{\prime}\right)}\right\} \\
V\left(C, \delta, \zeta^{\prime}\right)= & \left\{x \in \mathbb{C}^{n} ; C|x|<1,\left|\operatorname{Im} x^{\prime}\right|>C\left|x_{1}\right|^{1+\left(1 / m^{\prime}\right)},\right. \\
& \left|\frac{1}{\left|\operatorname{Im} x^{\prime}\right|} \operatorname{Im} x^{\prime}-\frac{1}{\left|\theta \zeta^{\prime o}+(1-\theta) \zeta^{\prime}\right|}\left(\theta \zeta^{\prime o}+(1-\theta) \zeta^{\prime}\right)\right|<\delta, \\
& 0 \leq \exists \theta \leq 1\}
\end{aligned}
$$

Let $0<C \ll C^{\prime} \ll C^{\prime \prime}$. If $u\left(x, y^{\prime}\right) \in \mathcal{O}(W(C, r)), f(x) \in \mathcal{O}(V(C, r))$, then

$$
u * f(x)=\int_{\Lambda\left(C^{\prime}, \varepsilon\right)} u\left(x, y^{\prime}\right) f\left(x_{1}, y^{\prime}\right) d\left(x^{\prime}-y^{\prime}\right) \in \mathcal{O}\left(V\left(C^{\prime \prime}, r\right)\right)
$$

is well-defined. Let $\mathscr{V}=\underset{C, r}{\lim } \mathcal{O}(V(C, r))$, let $\mathscr{V}_{1}, \mathscr{V}_{2}$ be defined similarly to $\mathscr{W}_{1}, \mathscr{W}_{2}$. We obtain a map $\mathscr{W} / \mathscr{W}_{2} \times \mathscr{V} / \mathscr{V}_{2} \ni(u, f) \mapsto u * f \in \mathscr{V} / \mathscr{V}_{2}$. In this way we can endow $\mathscr{V} / \mathscr{V}_{2}$ with the structure of a left $\mathscr{W} / \mathscr{W}_{2}$-module. If $a\left(x, \xi^{\prime}\right) \in \mathscr{T} / \mathscr{N}$, then $u=\mathscr{F}(a)$ defines an integral operator $\mathscr{V} / \mathscr{V}_{2} \ni f \mapsto$ $u * f \in \mathscr{V} / \mathscr{V}_{2}$, which we denote by $a\left(x, D^{\prime}\right)$. 
Remark. (i) Let $u\left(x, y^{\prime}\right) \in \mathcal{O}\left(W(C, r) \cup W\left(C, \delta, \zeta^{\prime}\right)\right), \quad f(x) \in \mathcal{O}\left(V\left(C, r^{\prime}\right)\right)$ with $0<r^{\prime} \leq r$. Then $u * f \in \mathscr{V}_{2}$, and this means $\int_{\Lambda\left(C^{\prime}, \varepsilon\right)} u\left(x, y^{\prime}\right) f\left(x_{1}, y^{\prime}\right)$. $d\left(x^{\prime}-y^{\prime}\right) \in \bigcup_{k: \text { finite }} \mathcal{O}\left(V\left(\exists C^{\prime \prime}, \exists \tilde{\delta}, \exists \tilde{\zeta}^{\prime(k)}\right)\right)$. However in the following special case this function is holomorphic in a full neighborhood of the origin. Assume that $r^{\prime}$ satisfies $\tilde{\zeta}_{n}^{(k)}+r^{\prime} \sum_{2 \leq j \leq n-1}\left|\tilde{\zeta}_{j}^{(k)}\right|<0$ for every $k$ (This is the case when $r^{\prime}$ is small enough). Let $\Lambda^{\prime}\left(C^{\prime}, \varepsilon\right)=\lambda_{2}^{\prime} \times \cdots \times \lambda_{n}^{\prime}$, where $\lambda_{k}^{\prime}$ is the union of line segments joining $-2 C^{\prime-1}-\sqrt{-1} \delta_{k, n} C^{\prime-3},-2 C^{\prime-1}+\sqrt{-1} R \zeta_{k}, 2 C^{\prime-1}+\sqrt{-1} R \zeta_{k}$, and $2 C^{\prime-1}-\sqrt{-1} \delta_{k, n} C^{\prime-3}$, successively $(0<R \ll 1)$. We can easily prove that

$$
\int_{\Lambda\left(C^{\prime}, \varepsilon\right)} u f d\left(x^{\prime}-y^{\prime}\right)=\int_{\Lambda^{\prime}\left(C^{\prime}, \varepsilon\right)} u f d\left(x^{\prime}-y^{\prime}\right) .
$$

Furthermore, if $x \in \mathbf{C}^{n},|x| \ll 1, x^{\prime}-y^{\prime} \in \Lambda^{\prime}\left(C^{\prime}, \varepsilon\right)$, then we have $\left(x, y^{\prime}\right) \in$ $W(C, r) \cup W\left(C, \delta, \zeta^{\prime}\right),\left(x_{1}, y^{\prime}\right) \in V\left(C, r^{\prime}\right)$. This means

$$
\int_{\Lambda^{\prime}\left(C^{\prime}, \varepsilon\right)} u f d\left(x^{\prime}-y^{\prime}\right) \in \mathcal{O}_{\mathbf{C}^{n}, 0}
$$

and therefore we have $u * f \in \mathcal{O}_{\mathbf{C}^{n}, 0}$.

(ii) As we have said $\mathscr{V} / \mathscr{V}_{2}$ is a left $\mathscr{W} / \mathscr{W}_{2}$-module. Therefore if $u_{1}, u_{2} \in \mathscr{W}$ and $f \in \mathscr{V}$, then we have $\left(u_{1} * u_{2}\right) * f \equiv u_{1} *\left(u_{2} * f\right)$ modulo $\mathscr{V}_{2}$. Furthermore, by an elementary calculation we can prove $\left(u_{1} * u_{2}\right) * f \equiv u_{1} *$ $\left(u_{2} * f\right)$ modulo $\mathcal{O}_{\mathbf{C}^{n}, 0}$.

All the above discussions are formal. However, if $u\left(x, y^{\prime}\right) \in \mathscr{W}$ (resp. $f(x) \in \mathscr{V})$, then $u\left(0, x^{\prime}, y^{\prime}\right)$ (resp. $f\left(0, x^{\prime}\right)$ ) defines a microfunction, and restricting to $\left\{x_{1}=0\right\}$, the above calculations are valid in the sense of microfunctions.

Remark. We sometimes consider functions with fractional powers in $x_{1}$. In this case we replace $\mathcal{O}$ by $\mathcal{O}_{\left(m^{\prime}\right)}$. We can generalize all the above arguments to this situation with trivial changes. For example let $\mathscr{T}_{\left(m^{\prime}\right)}=$ $\sum_{0 \leq k \leq m^{\prime}-1} x_{1}^{k / m^{\prime}} \mathscr{T}$, and $\mathscr{N}_{\left(m^{\prime}\right)}, \mathscr{W}_{\left(m^{\prime}\right)}, \mathscr{W}_{2,\left(m^{\prime}\right)}, \mathscr{V}_{\left(m^{\prime}\right)}, \mathscr{V}_{2,\left(m^{\prime}\right)}$ be defined similarly. Then $\mathscr{W}_{\left(m^{\prime}\right)} / \mathscr{W}_{2,\left(m^{\prime}\right)}$ is a ring, and $\mathscr{V}_{\left(m^{\prime}\right)} / \mathscr{V}_{2,\left(m^{\prime}\right)}$ is a left $\mathscr{W}_{\left(m^{\prime}\right)} / \mathscr{W}_{2,\left(m^{\prime}\right)}$-module. We sometimes differentiate $f=\sum_{0 \leq k \leq m^{\prime}-1} x_{1}^{k / m^{\prime}} f_{k} \in \mathcal{O}_{\left(m^{\prime}\right), x^{*}}$ by $x_{1}$, where $f_{k} \in \mathcal{O}_{x^{*}}$ $\left(0 \leq k \leq m^{\prime}-1\right)$. Since we do not necessarily have $\partial_{x_{1}} f \in \mathcal{O}_{\left(m^{\prime}\right), x^{*}}$, we must assume $\left.f_{k}\right|_{x_{1}=0}=0$ for $k \neq 0$, in this case.

\section{§2.2. A Theory of Real Operators}

We next consider how we can make the above discussions valid in the sense of microfunctions on the half space $\left\{x_{1} \gtrless 0\right\}$. This is a special case of the 
theory of mild microfunctions studied by [6]. Let $C>0, i \in \mathbb{Z}_{+}=\{0,1,2, \ldots\}$. Let $\theta \in\{0, \pi\}$. We define

$$
\begin{aligned}
& \Omega_{\theta}(C)=\left\{\left(x, \xi^{\prime}\right) \in \mathbb{C}^{n} \times \mathbb{C}^{n-1} ; C|x|<1, \operatorname{Re}\left(e^{\sqrt{-1} \theta} x_{1}\right)>0,\right. \\
&\left.C\left|\xi^{\prime \prime \prime}\right|<\operatorname{Im} \xi_{n}, C\left|\operatorname{Re} \xi_{n}\right|<\operatorname{Im} \xi_{n}, C^{4 m q}<\operatorname{Im} \xi_{n}\right\}, \\
& \Omega_{\theta, i}(C)=\left\{\left(x, \xi^{\prime}\right) \in \Omega_{\theta}(C) ; C^{4 m q}(i+1)<\operatorname{Im} \xi_{n}\right\} .
\end{aligned}
$$

Assume that for $\exists C>0, \exists R \in(0,1), \forall \varepsilon>0, \exists C_{\varepsilon}>0$, a formal series $f=$ $\sum_{i \in \mathbf{Z}_{+}} f_{i}\left(x, \xi^{\prime}\right) \in \mathscr{T}(\Omega(C))$ satisfies

$$
\begin{aligned}
\left|f_{i}\left(x, \xi^{\prime}\right)\right| \leq & C_{\varepsilon} R^{i} \exp \left(\left(C\left|x_{1}\right|^{1 / m^{\prime}}|\operatorname{Im} x|+C\left|x_{1}\right|^{1 / m^{\prime}}\left|\operatorname{Re} \xi^{\prime}\right| / \operatorname{Im} \xi_{n}\right.\right. \\
& \left.\left.+C\left(\left|\operatorname{Re} \xi^{\prime}\right| / \operatorname{Im} \xi_{n}\right)^{2}+\varepsilon\right) \operatorname{Im} \xi_{n}\right)
\end{aligned}
$$

on $\Omega_{\theta, i}(C)$ (Note that $f_{i} \in \mathcal{O}\left(\Omega_{i}(C)\right)$ satisfies (8) on $\Omega_{i}(C)$, and (13) on $\Omega_{\theta, i}(C)$ ). We denote by $\mathscr{S}_{\theta}(\Omega(C))$ the set of such formal series, and $\mathscr{S}_{\theta}=\underset{C>0}{\lim } \mathscr{S}_{\theta}(\Omega(C))$.

If $f=\sum f_{i}\left(x, \xi^{\prime}\right) \in \mathscr{S}_{\theta}(\Omega(C))$, we have $e f=\sum e\left(\tilde{\xi}^{\prime}\right) f_{i}\left(x, \xi^{\prime}\right) \in \mathscr{S}_{\theta}\left(\Omega\left(C^{\prime \prime}\right)\right)$ for some $C^{\prime \prime}$, and $\mathscr{F}(f)$ is holomorphic on $W\left(C^{\prime \prime \prime}, r\right) \cup W_{\theta}\left(C^{\prime \prime \prime}, r\right)$, where

$$
\begin{aligned}
& W_{\theta}\left(C^{\prime \prime \prime}, r\right)=\left\{\left(x, y^{\prime}\right) \in \mathbb{C}^{n} \times \mathbb{C}^{n-1} ; C^{\prime \prime \prime}\left|\left(x, y^{\prime}\right)\right|<1, \operatorname{Re}\left(e^{\sqrt{-1} \theta} x_{1}\right)>0,\right. \\
& \operatorname{Im}\left(x_{n}-y_{n}\right)+C^{\prime \prime \prime-1}\left(\left(\left|\operatorname{Re}\left(x^{\prime}-y^{\prime}\right)\right|-C^{\prime \prime \prime}\left|x_{1}\right|^{1 / m^{\prime}}\right)_{+}\right)^{2} \\
& \left.>C^{\prime \prime \prime}\left|x_{1}\right|^{1 / m^{\prime}}|\operatorname{Im} x|+r\left|\operatorname{Im}\left(x^{\prime \prime \prime}-y^{\prime \prime \prime}\right)\right|\right\}
\end{aligned}
$$

with $\exists C^{\prime \prime \prime}, \exists r$. Replacing the sheaf $\mathcal{O}$ by $\mathcal{O}_{\left(m^{\prime}\right)}$, we can define $\mathscr{S}_{\theta,\left(m^{\prime}\right)}$ similarly. If $f=\sum f_{i}\left(x, \xi^{\prime}\right) \in \mathscr{S}_{\theta,\left(m^{\prime}\right)}(\Omega(C))$, then we have $\mathscr{F}(f) \in \mathcal{O}_{\left(m^{\prime}\right)}\left(W_{\theta}\left(C^{\prime \prime \prime}, r\right)\right)$.

It is easy to see that if $u\left(x, y^{\prime}\right) \in \mathcal{O}_{\left(m^{\prime}\right)}\left(W(C, r) \cup W_{\theta}(C, r)\right)$, then it defines a microfunction $\operatorname{sp} u$ on $\left\{\operatorname{Re}\left(e^{\sqrt{-1} \theta} x_{1}\right)>0\right\}$. Note that $\operatorname{sp}\left(u\left(0, x^{\prime}, y^{\prime}\right)\right)$ is also well-defined, and it is a microlocal operator.

Let us define the corresponding operands. We define

$$
V_{\theta}(C, r)=\left\{x \in \mathbb{C}^{n} ; C|x|<1, \operatorname{Re}\left(e^{\sqrt{-1} \theta} x_{1}\right)>0, \operatorname{Im} x_{n}>r\left|\operatorname{Im} x^{\prime \prime}\right|\right\},
$$

where $C, r>0$. If $\mathcal{O}_{\left(m^{\prime}\right)}\left(V(C, r) \cup V_{\theta}(C, r)\right)$, then $f$ defines a microfunction $\operatorname{sp} f$ on $\left\{\operatorname{Re}\left(e^{\sqrt{-1} \theta} x_{1}\right)>0\right\}$. Moreover $\operatorname{sp} f$ is a mild microfunction in the sense of [6], and $\operatorname{sp}\left(f\left(0, x^{\prime}\right)\right)$ is well-defined.

If $u\left(x, y^{\prime}\right) \in \mathcal{O}_{\left(m^{\prime}\right)}\left(W(C, r) \cup W_{\theta}(C, r)\right), f(x) \in \mathcal{O}_{\left(m^{\prime}\right)}\left(V(C, r) \cup V_{\theta}(C, r)\right)$, then

$$
\begin{aligned}
u * f(x)= & \int_{\Lambda\left(C^{\prime}, \varepsilon\right)} u\left(x, y^{\prime}\right) f\left(x_{1}, y^{\prime}\right) d\left(x^{\prime}-y^{\prime}\right) \\
& \in \mathcal{O}_{\left(m^{\prime}\right)}\left(V\left(C^{\prime \prime}, r\right) \cup V_{\theta}\left(C^{\prime \prime}, r\right)\right)
\end{aligned}
$$


is well-defined $\left(C^{\prime \prime} \gg C^{\prime} \gg C\right)$. (14) coincides with the integration in the sense of microfunction on $\left\{\operatorname{Re}\left(e^{\sqrt{-1} \theta} x_{1}\right)>0\right\}$. Moreover, we can also restrict (14) to $\left\{x_{1}=0\right\}$ in the sense of microfunction.

\section{§ 2.3. A Theory of Annihilating Operators}

Let $\theta \in\{0, \pi\}$. Assume that for $\exists C>0, \exists R \in(0,1), \forall \varepsilon>0, \exists C_{\varepsilon}>0$, a formal series $f=\sum_{i \in \mathbf{Z}_{+}} f_{i}\left(x, \xi^{\prime}\right) \in \mathscr{T}(\Omega(C))$ satisfies

$$
\begin{aligned}
\left|f_{i}\left(x, \xi^{\prime}\right)\right| \leq & C_{\varepsilon} R^{i} \exp \left(\left(C\left|x_{1}\right|^{1 / m^{\prime}}|\operatorname{Im} x|+C\left|x_{1}\right|^{1 / m^{\prime}}\left|\operatorname{Re} \xi^{\prime}\right| / \operatorname{Im} \xi_{n}\right.\right. \\
& \left.\left.+C\left(\left|\operatorname{Re} \xi^{\prime}\right| / \operatorname{Im} \xi_{n}\right)^{2}-C^{-1}\left|x_{1}\right|^{1+\left(1 / m^{\prime}\right)}+\varepsilon\right) \operatorname{Im} \xi_{n}\right)
\end{aligned}
$$

on $\Omega_{\theta, i}(C)$. Then $\mathscr{F}(f)$ is holomorphic on $W\left(C^{\prime \prime \prime}, r\right) \cup W_{\theta}^{\prime}\left(C^{\prime \prime \prime}, r\right)$ with $\exists C^{\prime \prime \prime}, \exists r$, where

$$
\begin{aligned}
& W_{\theta}^{\prime}\left(C^{\prime \prime \prime}, r\right)=\left\{\left(x, y^{\prime}\right) \in \mathbf{C}^{n} \times \mathbf{C}^{n-1} ; C^{\prime \prime \prime}\left|\left(x, y^{\prime}\right)\right|<1, \operatorname{Re}\left(e^{\sqrt{-1} \theta} x_{1}\right)>0\right. \\
& \left.\operatorname{Im}\left(x_{n}-y_{n}\right)+C^{\prime \prime \prime-1}\left|x_{1}\right|^{1+\left(1 / m^{\prime}\right)}>C^{\prime \prime \prime}\left|x_{1}\right|^{1 / m^{\prime}}|\operatorname{Im} x|+r\left|\operatorname{Im}\left(x^{\prime \prime \prime}-y^{\prime \prime \prime}\right)\right|\right\} .
\end{aligned}
$$

Let

$$
\begin{array}{r}
V_{\theta}^{\prime}(C, r)=\left\{x \in \mathbf{C}^{n} ; C|x|<1, \operatorname{Re}\left(e^{\sqrt{-1} \theta} x_{1}\right)>0\right. \\
\left.\operatorname{Im} x_{n}+C^{-1}\left|x_{1}\right|^{1+\left(1 / m^{\prime}\right)}>r\left|\operatorname{Im} x^{\prime \prime}\right|\right\} .
\end{array}
$$

If $u \in \mathcal{O}\left(W_{\theta}^{\prime}(C, r)\right)$ (resp. $\left.f \in \mathcal{O}\left(V_{\theta}^{\prime}(C, r)\right)\right)$, then $\operatorname{sp} u=0$ (resp. $\operatorname{sp} f=0$ ) on $\left\{\operatorname{Re}\left(e^{\sqrt{-1} \theta} x_{1}\right)>0\right\}$. If $u \in \underset{C, r}{\lim } \mathcal{O}\left(W_{\theta}^{\prime}(C, r)\right)$ or $f \in \underset{C, r}{\lim } \mathcal{O}\left(V_{\theta}^{\prime}(C, r)\right)$, then we have $u * f \in \underset{C, r}{\lim } \mathcal{O}\left(V_{\theta}^{\prime}(C, r)\right)$. Of course we can consider the case of fractional powers in $x_{1}$.

\section{§ 2.4. Other Symbol Classes}

Sometimes it is important to consider a formal series defined only for the case when $x_{1}$ belongs to a sector. Let $\theta \in[0,2 \pi]=\{t \in \mathbf{R} ; 0 \leq t \leq 2 \pi\}$, and let

$$
\begin{gathered}
\Omega(C, \theta)=\left\{\left(x, \xi^{\prime}\right) \in \Omega(C) ; x_{1} \neq 0, C\left|\arg x_{1}-\theta\right|<1\right\} \\
\Omega_{i}(C, \theta)=\Omega(C, \theta) \cap \Omega_{i}(C)
\end{gathered}
$$

(Here we do not restrict $\theta$ to $\{0, \pi\})$. We define $\mathscr{T}^{\theta}(\Omega(C, \theta)$ ) (resp. $\left.\mathscr{S}^{\theta}(\Omega(C, \theta))\right)$ as the set of formal series $f=\sum_{i \in \mathbf{Z}_{+}} f_{i}\left(x, \xi^{\prime}\right)$ satisfying (8) (resp. (13)) on $\Omega_{i}(C, \theta)$, instead of $\Omega_{i}(C)$. We define $\mathscr{R}^{\theta}(\Omega(C, \theta))$ as the set of formal 
series $\sum_{i} f_{i} \in \mathscr{T}^{\theta}(\Omega(C))$ such that for $\exists R \in(0,1), \forall \varepsilon>0, \exists C_{\varepsilon}>0$ we have $\left|f_{i}\left(x, \xi^{\prime}\right)\right| \leq C_{\varepsilon} R^{i} \exp \left(\varepsilon \operatorname{Im} \xi_{n}\right)$ on $\Omega_{i}(C, \theta)$. We define $\mathscr{R}^{\theta}=\underset{C>0}{\lim _{\longrightarrow}} \mathscr{R}^{\theta}(\Omega(C, \theta))$, and similarly we define $\mathscr{S}^{\theta}, \mathscr{T}^{\theta}$.

Lemma 4. Let $\theta \in\{0, \pi\}$. We have $\mathscr{T} \cap \mathscr{S}^{\theta} \subset \mathscr{S}_{\theta}$ and $\mathscr{T}_{\left(m^{\prime}\right)} \cap \mathscr{S}^{\theta} \subset$ $\mathscr{S}_{\theta,\left(m^{\prime}\right)}$.

Proof. Let $\sum f_{i} \in \mathscr{T} \cap \mathscr{S}^{\theta}$. Let $\varepsilon$ be an arbitrary number. If $\left(x, \xi^{\prime}\right) \in$ $\Omega_{\theta, i}(C)$ satisfies $\left|\operatorname{Im} x_{1}\right| \ll\left|\operatorname{Re} x_{1}\right|$, we have

$$
\begin{aligned}
\left|f_{i}\left(x, \xi^{\prime}\right)\right| \leq & \exists C_{\varepsilon} \exists R^{i} \exp \left\{C \left(\left|x_{1}\right|^{1 / m^{\prime}}|\operatorname{Im} x|+C\left|x_{1}\right|^{1 / m^{\prime}}\left|\operatorname{Re} \xi^{\prime}\right|\left|\operatorname{Im} \xi_{n}\right|^{-1}\right.\right. \\
& \left.\left.+C\left|\operatorname{Re} \xi^{\prime}\right|^{2}\left|\operatorname{Im} \xi_{n}\right|^{-2}+\forall \varepsilon\right) \operatorname{Im} \xi_{n}\right\} .
\end{aligned}
$$

If $\left(x, \xi^{\prime}\right) \in \Omega_{\theta, i}(C)$ does not satisfy $\left|\operatorname{Im} x_{1}\right| \ll\left|\operatorname{Re} x_{1}\right|$, we have

$$
\begin{aligned}
\left|f_{i}\left(x, \xi^{\prime}\right)\right| & \leq \exists C_{\varepsilon} \exists R^{i} \exp \left(\left(C\left|x_{1}\right|^{1+\left(1 / m^{\prime}\right)}+C\left(\left|\operatorname{Re} \xi^{\prime}\right| / \operatorname{Im} \xi_{n}\right)^{2}+\varepsilon\right) \operatorname{Im} \xi_{n}\right) \\
& \left.\leq C_{\varepsilon} R^{i} \exp \left(\exists C^{\prime}\left|x_{1}\right|^{1 / m^{\prime}}|\operatorname{Im} x|+C\left(\left|\operatorname{Re} \xi^{\prime}\right| / \operatorname{Im} \xi_{n}\right)^{2}+\varepsilon\right) \operatorname{Im} \xi_{n}\right) .
\end{aligned}
$$

This means $\sum f_{i} \in \mathscr{S}_{\theta}$. The proof of the latter statement is the same. Q.E.D.

\section{§3. Transformation by Holomorphic Microlocal Operators}

In this section, we given a canonical representation of the elementary solution of the Cauchy problem (5). We first rewrite the equation using matrices. Let $L(x, D)$ be an $m \times m$ matrix defined by $L(x, D)=D_{1} I_{m}+$ $\bar{L}\left(x, D^{\prime}\right)$, where $I_{m}$ is the unit matrix and $\bar{L}$ is defined by

$$
\bar{L}=\left(\begin{array}{cccc}
0, & -1, & & 0 \\
& \ddots & \ddots & \\
0 & & 0, & -1, \\
P_{0}\left(x, D^{\prime}\right), & P_{1}\left(x, D^{\prime}\right), & \ldots, & P_{m-1}\left(x, D^{\prime}\right)
\end{array}\right) .
$$

Let $\quad \vec{u}(x)={ }^{t}\left(u, D_{1} u, \ldots, D_{1}^{m-1} u\right) \in\left(\mathscr{C}_{\mathbf{R}^{n}, x^{*}}\right)^{m} \quad$ and $\quad \vec{v}\left(x^{\prime}\right)={ }^{t}\left(v_{1}, \ldots, v_{m}\right) \in$ $\left(\mathscr{C}_{\mathbf{R}^{n-1}, x^{* \prime \prime}}\right)^{m}$. Then (5) is equivalent to

$$
L \vec{u}=\overrightarrow{0}, \quad \vec{u}\left(0, x^{\prime}\right)=\vec{v}\left(x^{\prime}\right) .
$$

We grade the complete symbol of $\bar{L}$ as follows. If $A(x, D)=\sum_{j \leq \ell} A_{j}(x, D) \in \mathscr{E}_{x^{*}}$ is a microdifferential operator of order at most $\ell \in \mathbb{Z}$, and each $A_{j}$ is homogeneous in $D$ of degree $j$, we denote $A_{j}(x, \xi)$ by $\sigma_{j}(A)$ (This notation depends on the choice of the symplectic coordinate system, except for the principal symbol). Now we define 


$$
\bar{L}_{j}=\left(\begin{array}{cccc}
0, & -\delta_{j 0}, & & 0 \\
0 & \ddots & \ddots & \\
\sigma_{m-j}\left(P_{0}\right), & \sigma_{m-j-1}\left(P_{1}\right), & \ldots, & \sigma_{1-j}\left(P_{m-1}\right)
\end{array}\right)
$$

for $j \in \mathbb{Z}_{+}$. If $Y$ is a ring, we denote by $Y^{m \times m}$ the ring of $m \times m$ matrices whose components belong to $Y$. It is easy to see that we can inductively define $E_{i}^{( \pm)}(x, \xi) \in(\mathcal{O}(\Omega(C)))^{m \times m}, i \in \mathbf{Z}_{+}, C \gg 1$, by

$$
\begin{cases}\partial_{x_{1}} E^{(+)}\left(x, \xi^{\prime}\right)+\bar{L}\left(x, \xi^{\prime}\right) \circ E^{(+)}=O, & E^{(+)}\left(0, x^{\prime}, \xi^{\prime}\right)=1, \\ \partial_{x_{1}} E^{(-)}\left(x, \xi^{\prime}\right)-E^{(-)} \circ \bar{L}\left(x, \xi^{\prime}\right)=O, & E^{(-)}\left(0, x^{\prime}, \xi^{\prime}\right)=1\end{cases}
$$

where $E^{( \pm)}=\sum_{i} E_{i}^{( \pm)}$. In fact, if $E_{i^{\prime}}^{( \pm)}$are already calculated for $0 \leq i^{\prime} \leq$ $i-1,(15)$ is an ordinary differential equation for $E_{i}^{( \pm)}$, which is easy to solve. For each number $\lambda \in\{1, \ldots, m\}$, let $X^{( \pm, \lambda)}\left(x, D^{\prime}\right)$ be a Fourier integral operator (maybe with a complex phase function) satisfying

$$
\partial_{x_{1}} X^{(+, \lambda)}\left(x, \xi^{\prime}\right)-\varphi_{\lambda}\left(x, \xi^{\prime}\right) \circ X^{(+, \lambda)}\left(x, \xi^{\prime}\right)=0, \quad X^{(+, \lambda)}\left(0, x^{\prime}, \xi^{\prime}\right)=1,
$$

and

$$
\partial_{x_{1}} X^{(-, \lambda)}\left(x, \xi^{\prime}\right)+X^{(-, \lambda)}\left(x, \xi^{\prime}\right) \circ \varphi_{\lambda}\left(x, \xi^{\prime}\right)=0, \quad X^{(-, \lambda)}\left(0, x^{\prime}, \xi^{\prime}\right)=1,
$$

respectively.

Remark. Here we are considering holomorphic functions in $\left(x_{1}^{1 / m^{\prime}}, x^{\prime}, \xi^{\prime}\right)$. If $f=\sum_{0 \leq k \leq m^{\prime}-1} x_{1}^{k / m^{\prime}} f_{k} \in \mathcal{O}_{\left(m^{\prime}\right), x^{*}}$ with $f_{k} \in \mathcal{O}_{x^{*}}$, then we define $\left.f\right|_{x_{1}=0}=\left.f_{0}\right|_{x_{1}=0}$. We consider the above equations in this sense.

In fact we can calculate the complete symbol $X^{( \pm, \lambda)}\left(x, \xi^{\prime}\right)$ of the corresponding operator in the form $X^{( \pm, \lambda)}\left(x, \xi^{\prime}\right) \equiv \exp \left(\psi^{( \pm, \lambda)}\left(x, \xi^{\prime}\right)\right) \sum_{i} X_{i}^{\prime( \pm, \lambda)}\left(x, \xi^{\prime}\right)$ modulo $\mathscr{N}_{\left(m^{\prime}\right)}(\Omega(C))$ with some elliptic amplitude function $\sum_{i} X_{i}^{\prime( \pm, \lambda)}$ of order 0 . Here we denote by $\psi^{( \pm, \lambda)}\left(x, \xi^{\prime}\right)$ the phase function defined by $(6)_{ \pm}$. Let $X^{( \pm)}\left(x, \xi^{\prime}\right)$ be the matrix defined by

$$
X^{( \pm)}\left(x, \xi^{\prime}\right)=\left(\begin{array}{ccc}
X^{( \pm, 1)} & & 0 \\
& \ddots & \\
0 & & X^{( \pm, m)}
\end{array}\right) \in \mathscr{T}_{\left(m^{\prime}\right)}^{m \times m} .
$$

Now we have the following

Theorem 2. We assume (2), (3), (4), and (7). We have $E^{( \pm)}\left(x, \xi^{\prime}\right) \in$ $\mathscr{T}^{m \times m}$, and for each $\theta \in[0,2 \pi]$ there exist $F^{( \pm, \theta)}\left(x, \xi^{\prime}\right), \quad G^{( \pm, \theta)}\left(x^{\prime}, \xi^{\prime}\right) \in$ $\left(\mathscr{T}_{\left(m^{\prime}\right)} \cap \mathscr{R}^{\theta}\right)^{m \times m}$ such that 


$$
\begin{gathered}
E^{(+, \theta)}\left(x, \xi^{\prime}\right)=F^{(+, \theta)}\left(x, \xi^{\prime}\right) \circ X^{(+)}\left(x, \xi^{\prime}\right) \circ G^{(-, \theta)}\left(x^{\prime}, \xi^{\prime}\right), \\
E^{(-, \theta)}\left(x, \xi^{\prime}\right)=G^{(+, \theta)}\left(x^{\prime}, \xi^{\prime}\right) \circ X^{(-)}\left(x, \xi^{\prime}\right) \circ F^{(-, \theta)}\left(x, \xi^{\prime}\right), \\
F^{( \pm, \theta)}\left(x, \xi^{\prime}\right) \circ F^{(\mp, \theta)}\left(x, \xi^{\prime}\right)=I_{m}, \\
G^{( \pm, \theta)}\left(x^{\prime}, \xi^{\prime}\right) \circ G^{(\mp, \theta)}\left(x^{\prime}, \xi^{\prime}\right)=I_{m}, \\
F^{( \pm, \theta)}\left(0, x^{\prime}, \xi^{\prime}\right)=G^{( \pm, \theta)}\left(x^{\prime}, \xi^{\prime}\right)
\end{gathered}
$$

if $\left(x, \xi^{\prime}\right) \in \Omega(C, \theta)$.

Remark. (i) Note that $\theta$ is an arbitrary real number belonging to $[0,2 \pi]$ this time.

(ii) $F^{( \pm, \theta)}$ is defined in a full neighborhood of $x_{1}=0$, belongs to $\mathscr{T}_{\left(m^{\prime}\right)}^{m \times m}$ there, and belongs to $\left(\mathscr{R}^{\theta}\right)^{m \times m}$ in a sector around $x_{1}=0$. Therefore $F^{( \pm, \theta)}\left(0, x^{\prime}, \xi^{\prime}\right)$ is well-defined.

(iii) Since $G^{( \pm, \theta)}=\sum_{i \geq 0} G^{( \pm, \theta)}$ does not depend on $x_{1}$, it follows that its $(\mu, v)$-element $G_{i,(\mu, v)}^{( \pm, \theta)}$ is holomorphic on $\mathcal{O}\left(\Omega_{i}(C)\right)$, and $\exists R \in(0,1), \forall \varepsilon>0$, $\exists C_{\varepsilon}>0$ we have $\left|G_{i,(\mu, v)}^{( \pm, \theta)}\left(x^{\prime}, \xi^{\prime}\right)\right| \leq C_{\varepsilon} R^{i} \exp \left(\varepsilon \operatorname{Im} \xi_{n}\right)$ on $\Omega_{i}(C)$. According to [2], it is a symbol of $G_{(\mu, v)}^{( \pm, \theta)}\left(x^{\prime}, D^{\prime}\right) \in \mathscr{E}_{x^{*}}^{\mathbb{R}}$, and we have $G^{( \pm, \theta)}\left(x^{\prime}, D^{\prime}\right) G^{(\mp, \theta)}\left(x^{\prime}, D^{\prime}\right)$ $=I_{m}$.

(iv) $E^{( \pm)}\left(x, D^{\prime}\right), X^{( \pm)}\left(x, D^{\prime}\right), F^{( \pm, \theta)}\left(x, D^{\prime}\right), G^{( \pm, \theta)}\left(x^{\prime}, D^{\prime}\right)$ are operators acting on $\left(\mathscr{V}_{\left(m^{\prime}\right)} / \mathscr{V}_{2,\left(m^{\prime}\right)}\right)^{m}$. Some of these operators (and their composites) do not contain fractional powers in $x_{1}$.

(v) Let $\theta \in\{0, \pi\}$. Then $F^{( \pm, \theta)}\left(x, D^{\prime}\right)$ is a map of $\left(\mathcal{O}_{\left(m^{\prime}\right)}(V(C, r) \cup\right.$ $\left.\left.V_{\theta}(C, r)\right)\right)^{m}$ into $\left(\mathcal{O}_{\left(m^{\prime}\right)}\left(V\left(C^{\prime}, r\right) \cup V_{\theta}\left(C^{\prime}, r\right)\right)\right)^{m}$ with $C^{\prime} \gg C$.

(vi) Let $\theta \in\{0, \pi\}$. If $\mu \in M_{0, \theta} \cup M_{-, \theta}$ (resp. $\mu \in M_{0, \theta} \cup M_{+, \theta}$ ), then $X_{(\mu, \mu)}^{(+)}\left(\right.$resp. $\left.X_{(\mu, \mu)}^{(-)}\right)$is a map of $\mathcal{O}_{\left(m^{\prime}\right)}\left(V(C, r) \cup V_{\theta}(C, r)\right)$ into $\mathcal{O}_{\left(m^{\prime}\right)}\left(V\left(C^{\prime}, r\right) \cup\right.$ $\left.V_{\theta}\left(C^{\prime}, r\right)\right)$ with $C^{\prime} \gg C$. This operator is well-defined in the sense of microfunctions on $\left\{e^{\sqrt{-1} \theta} x_{1}>0\right\}$.

(vii) Let $\theta \in\{0, \pi\}$. If $\mu \in M_{-, \theta}$ (resp. $\mu \in M_{+, \theta}$ ), then $X_{(\mu, \mu)}^{(+)}\left(\right.$resp. $\left.X_{(\mu, \mu)}^{(-)}\right)$ is a map of $\mathcal{O}\left(V(C, r) \cup V_{\theta}(C, r)\right)$ into $\mathcal{O}_{\left(m^{\prime}\right)}\left(V\left(C^{\prime}, r\right) \cup V_{\theta}^{\prime}\left(C^{\prime}, r\right)\right)$ with $C^{\prime} \gg C$. This operator annihilates all the microfunctions on $\left\{e^{\sqrt{-1} \theta} x_{1}>0\right\}$.

Admitting Theorem 2, we can prove Theorem 1 as follows. We want to prove that the Cauchy problem is solvable if, and only if, $v_{1}\left(x^{\prime}\right), \ldots, v_{m}\left(x^{\prime}\right) \in$ $\mathscr{C}_{\mathbf{R}^{n-1}, x^{* \prime}}$ satisfy the following $m_{+, 0}$-relation:

$$
\left(G^{(-, 0)}\left(x^{\prime}, D^{\prime}\right) \vec{v}\left(x^{\prime}\right)\right)_{\mu}=0, \quad \mu \in M_{+, 0},
$$

and the following $m_{+, \pi}$-relation:

$$
\left(G^{(-, \pi)}\left(x^{\prime}, D^{\prime}\right) \vec{v}\left(x^{\prime}\right)\right)_{\mu}=0, \quad \mu \in M_{+, \pi} .
$$


Let us prove the sufficiency. We want to show that if $\vec{v}$ satisfies $(16)_{0}$ and $(16)_{\pi}$, then $E^{(+)}\left(x, D^{\prime}\right) \vec{v}$ is well-defined as a microfunction and its first component $\left(E^{(+)}\left(x, D^{\prime}\right) \vec{v}\right)_{1}$ satisfies $(5)$.

Let $\theta \in\{0, \pi\}$, and let $\vec{w}^{(\theta)}=G^{(-, \theta)}\left(x^{\prime}, D^{\prime}\right) \vec{v}\left(x^{\prime}\right)$. We assume $(16)_{\theta}$. Therefore we have $\vec{w}_{\mu}^{\theta}=0$ for $\mu \in M_{+, \theta}$, and $\vec{v}\left(x^{\prime}\right)=G^{(+, \theta)}\left(x^{\prime}, D^{\prime}\right) \vec{w}^{\theta}\left(x^{\prime}\right)$. We have

$$
\nu \notin M_{+, \theta} \Rightarrow\left\{\begin{array}{l}
\left(E^{(+)}\left(x, \xi^{\prime}\right) \circ G^{(+, \theta)}\left(x^{\prime}, \xi^{\prime}\right)\right)_{(\mu, v)} \in \mathscr{T} \cap \mathscr{S}^{\theta} \subset \mathscr{S}_{\theta}, \\
\left(\mathscr{F}\left(E^{(+)} \circ G^{(+, \theta)}\right)\right)_{(\mu, v)}\left(x, y^{\prime}\right) \in \mathcal{O}\left(W(C, r) \cup W_{\theta}(C, r)\right), \quad \exists C, \exists r .
\end{array}\right.
$$

Let $\vec{v}^{\sim}$ be the defining function of $\vec{v}$. We may assume that supp $\vec{v}$ is small, and therefore we have $\vec{v}^{\sim} \in \mathcal{O}(V(C, r))^{m}$, where $r$ is small enough. We can define $\vec{u}^{\sim}={ }^{t}\left(u_{1}^{\sim}, \ldots, u_{m}^{\sim}\right) \in \mathscr{V}^{m}$ by $\vec{u}^{\sim}=\mathscr{F}\left(E^{(+)}\right) * \vec{v}^{\sim}$. Then we have

$$
\begin{aligned}
u_{\mu}^{\sim}(x) & =\left(\mathscr{F}\left(E^{(+)}\right) * \vec{v}^{\sim}\right)_{\mu} \equiv \sum_{1 \leq \kappa \leq m}\left(\mathscr{F}\left(E^{(+)}\right) * \mathscr{F}\left(G^{(+, \theta)}\right)\right)_{(\mu, \kappa)} * \vec{w}_{\kappa}^{(\theta) \sim}\left(x^{\prime}\right) \\
& \equiv \sum_{\kappa \notin M_{+, \theta}}\left(\mathscr{F}\left(F^{(+, \theta)}\right) * \mathscr{F}\left(X^{(+)}\right)\right)_{(\mu, \kappa)} * \vec{w}_{\kappa}^{(\theta) \sim}\left(x^{\prime}\right) \text { modulo } \mathcal{O}_{\mathbf{C}^{n}, 0}
\end{aligned}
$$

for any $\mu$. It follows that $\vec{u}^{\sim}(x) \in \mathcal{O}\left(V(C, r) \cup V_{0}(C, r) \cup V_{\pi}(C, r)\right)^{m}$. We have $\mathscr{F}(L) * \vec{u}^{\sim}(x) \equiv \mathscr{F}(L) * \mathscr{F}\left(E^{(+)}\right) * \vec{v}^{\sim}\left(x^{\prime}\right) \equiv \mathscr{F}\left(E^{(+)}\right) *\left(D_{1} \vec{v}^{\sim}\left(x^{\prime}\right)\right)=\overrightarrow{0}$ modulo $\mathcal{O}_{\mathbf{C}^{n}, 0}^{m}$. This means $\vec{u} \in\left(\mathscr{C}_{\mathbf{R}^{n}, x^{*}}\right)^{m}, L(x, D) \vec{u}=\overrightarrow{0}, \vec{u}\left(0, x^{\prime}\right)=\vec{v}\left(x^{\prime}\right)$, and $u_{1}$ satisfies (5).

We next prove the necessity. Let $\theta \in\{0, \pi\}$. We want to show that if $\mu \in M_{+, \theta}$, then $\left(G^{(-, \theta)}\left(x^{\prime}, D^{\prime}\right) E^{(-)}\left(x, D^{\prime}\right) \vec{v}\right)_{\mu}$ is a microfunction which does not depend on $x_{1}$, coincides with $\left(G^{(-, \theta)}\left(x^{\prime}, D^{\prime}\right) \vec{v}\right)_{\mu}$ if $x_{1}=0$, and vanishes if $e^{\sqrt{-1} \theta} x_{1}$ $>0$ (Note that this means $\left.(16)_{0},(16)_{\pi}\right)$.

We assume that $u$ is a solution of (5). We define $\vec{u}={ }^{t}\left(u_{1}, \ldots, u_{m}\right)$ by $u_{j}=$ $D_{1}^{j-1} u$, and therefore $\vec{u}$ satisfies $L(x, D) \vec{u}=\overrightarrow{0}, \vec{u}\left(0, x^{\prime}\right)=\vec{v}\left(x^{\prime}\right)={ }^{t}\left(v_{1}, \ldots, v_{m}\right)$. We define

$$
\vec{U}= \begin{cases}\vec{u}, & \left|x^{\prime}\right|<R,\left|\operatorname{Im} \xi^{\prime \prime \prime}\right|<R \operatorname{Im} \xi_{n}, \\ \overrightarrow{0}, & \left|\operatorname{Im} \xi^{\prime \prime}\right|>R \operatorname{Im} \xi_{n}, \\ \overrightarrow{0}, & \left|x^{\prime}\right|>R\end{cases}
$$

where $R>0$ is small enough. Let $\vec{V}\left(x^{\prime}\right)=\vec{U}\left(0, x^{\prime}\right)$. Then we have $\operatorname{supp}(L(x, D) \vec{U}) \subset\left\{\left(x, \xi^{\prime}\right) ;\left|x^{\prime}\right|=R\right\} \cup\left\{\left|\operatorname{Im} \xi^{\prime \prime \prime}\right|=R \operatorname{Im} \xi_{n}\right\}, \quad$ and $\vec{V}\left(x^{\prime}\right)=\vec{v}\left(x^{\prime}\right)$ on $\left\{\left(x^{\prime}, \xi^{\prime}\right) ;\left|x^{\prime}\right|<R,\left|\operatorname{Im} \xi^{\prime \prime \prime}\right|<R \operatorname{Im} \xi_{n}\right\}$, and $\operatorname{supp} \vec{V}\left(x^{\prime}\right) \subset\left\{\left(x^{\prime}, \xi^{\prime}\right) ;\left|x^{\prime}\right| \leq R\right.$, $\left.\left|\operatorname{Im} \xi^{\prime \prime \prime}\right| \leq R \operatorname{Im} \xi_{n}\right\}$. Let $K=\left(K_{(\mu, v)}\right) \in \mathbf{C}^{m \times m}$ be the diagonal matrix defined by $K_{(\mu, \mu)}^{\theta}=0$ if $\mu \notin M_{+, \theta}$, and $K_{(\mu, \mu)}^{\theta}=1$ if $\mu \in M_{+, \theta}$. We may assume that $\vec{U}$ has a defining function $\vec{U}^{\sim} \in(\mathcal{O}(V(C, r)))^{m}$ with $0<r \ll 1$. Let $\vec{W}^{(\theta) \sim}=$ $\left(K G^{(-, \theta)} * E^{(-)}\right) * \vec{U}^{\sim}$, and let $\vec{V}^{\sim}\left(x^{\prime}\right)=\vec{U}^{\sim}\left(0, x^{\prime}\right)$. We have 


$$
\begin{aligned}
D_{1} \vec{W}^{(\theta)}(x) & \equiv D_{1}\left(K G^{(-, \theta)} * E^{(-)} * \vec{U}^{\sim}\right) \\
& \equiv\left(K G^{(-, \theta)} * E^{(-)}\right) *\left(\mathscr{F}(L) * \vec{U}^{\sim}\right) \quad \text { modulo } \mathcal{O}_{\mathbf{C}^{n}, 0}^{m} .
\end{aligned}
$$

Here the right-hand side belongs to $\mathscr{V}_{2}^{m}$. Furthermore, it defines a hyperfunction, which is microanalytic at $d x_{n} \infty$ on $\left\{e^{\sqrt{-1} \theta} x_{1}>0\right\}$. We also have

$$
\vec{W}^{(\theta) \sim}\left(0, x^{\prime}\right) \equiv K G^{(-, \theta)} * \vec{U}^{\sim}\left(0, x^{\prime}\right) \equiv K G^{(-, \theta)} * \vec{V}^{\sim}\left(x^{\prime}\right) \quad \text { modulo } \mathcal{O}_{\mathbf{C}^{n}, 0}^{m} .
$$

It follows that $\vec{W}^{(\theta)} \sim(x) \equiv \vec{W}^{(\theta) \sim}\left(0, x^{\prime}\right)$ modulo $\mathscr{V}_{2}^{m}$. We have $K G^{(-, \theta)} * E^{(-)} \in$ $\mathcal{O}\left(W_{\theta}^{\prime}\left(C^{\prime}, r^{\prime}\right)\right)^{m \times m}$ with some $\exists C^{\prime}>0, \quad \exists r^{\prime}>0$, and $\vec{W}^{(\theta)} \sim \mathcal{O}\left(V_{\theta}^{\prime}\left(C^{\prime \prime}, r^{\prime \prime}\right)\right)^{m}$ with some $\exists C^{\prime \prime}>0, \exists r^{\prime \prime}>0$. If $\bar{x}_{1}$ is a constant satisfying $e^{\sqrt{-1} \theta} \bar{x}_{1}>0$, then $\vec{W}^{(\theta)} \sim\left(\bar{x}_{1}, x^{\prime}\right)$ is analytic at $x^{\prime}=0$, and we obtain $\vec{W}^{(\theta)} \sim\left(0, x^{\prime}\right) \in \mathscr{V}_{2}^{m}$. Taking the singularity spectrum we obtain $(16)_{\theta}$.

Therefore we next need to prove Theorem 2, and the plan is as follows. We define $\bar{M}\left(x, \xi^{\prime}\right)$ by

$$
\bar{M}\left(x, \xi^{\prime}\right)=\left(\begin{array}{ccc}
-\varphi_{1}\left(x, \xi^{\prime}\right) & & 0 \\
& \ddots & \\
0 & & -\varphi_{m}\left(x, \xi^{\prime}\right)
\end{array}\right)
$$

It suffices to find $F^{( \pm, \theta)}\left(x, \xi^{\prime}\right) \in\left(\mathscr{T}_{\left(m^{\prime}\right)} \cap \mathscr{R}^{\theta}\right)^{m \times m}$ satisfying

$$
\left\{\begin{array}{l}
\partial_{x_{1}} F^{(+, \theta)}+\bar{L} \circ F^{(+, \theta)}-F^{(+, \theta)} \circ \bar{M}=O, \\
\partial_{x_{1}} F^{(-, \theta)}+\bar{M} \circ F^{(-, \theta)}-F^{(-, \theta)} \circ \bar{L}=O, \\
F^{( \pm, \theta)} \circ F^{(\mp, \theta)}=I_{m} .
\end{array}\right.
$$

For this purpose we need to consider the case $\left|x_{1} \xi_{n}\right| \gg 1$ and the contrary case separately. Precisely speaking, let $C \gg 1, \theta \in[0,2 \pi]$, and we define

$$
\begin{aligned}
& \Xi(C)=\left\{\left(x, \xi^{\prime}\right) \in \mathbb{C}^{n} \times \mathbb{C}^{n-1} ; C|x|<1, C\left|\xi^{\prime \prime \prime}\right|<\operatorname{Im} \xi_{n},\right. \\
&\left.C\left|\operatorname{Re} \xi_{n}\right|<\operatorname{Im} \xi_{n}, C^{4 m(q+1)}<\operatorname{Im} \xi_{n}\right\}, \\
& \Xi^{\prime}(C)=\left\{\left(x, \xi^{\prime}\right) \in \Xi(C) ; C\left(\operatorname{Im} \xi_{n}\right)^{-1 /(m q+m)}<\left|x_{1}\right|\right\}, \\
& \Xi^{\prime}(C, \theta)=\left\{\left(x, \xi^{\prime}\right) \in \Xi^{\prime}(C) ; C\left|\arg x_{1}-\theta\right|<1\right\}, \\
& \Xi^{\prime \prime}(C)=\left\{\left(x, \xi^{\prime}\right) \in \Xi(C) ;\left|x_{1}\right|<C^{-1 / 2}\left(\operatorname{Im} \xi_{n}\right)^{-1 /(2 m q+2 m)}\right\} .
\end{aligned}
$$

Note that $\Xi^{\prime}(C) \cup \Xi^{\prime \prime}(C)=\Xi(C)$.

At first we shall calculate $F^{( \pm, \theta)}$ on $\Xi^{\prime}(C)$. This part is divided into several steps. Section 4 is an auxiliary step. The most important part is Section 6, and in Section 5 we shall prepare an estimate of the phase functions, which will be necessary in Section 6 . Since such a phase calculation is valid when $x_{1}$ belongs to a sector with its vertex at the origin, we shall discuss on $\Xi^{\prime}(C, \theta)$. 
After that we shall calculate $F^{( \pm, \theta)}$ on $\Xi^{\prime \prime}(C)$ in Section 7, and also on the whole $\Xi(C)$ (We do not divide $\Xi^{\prime \prime}(C)$ into sectors). Let us illustrate our plan by a special case without proof.

Example. For the sake of simplicity we assume that $m=2$ and let $L=$ $D_{1} I_{2}+\bar{L}\left(x_{1}, D_{n}\right)$, where

$$
\bar{L}\left(x_{1}, D_{n}\right)=\left(\begin{array}{cc}
0 & -1 \\
-x_{1}^{2 q} D_{n}^{2}+a D_{n} & b
\end{array}\right), \quad a, b \in \mathbf{C}
$$

We need to solve

$$
\begin{cases}\partial_{x_{1}} E^{(+)}+\bar{L}\left(x_{1}, \xi_{n}\right) E^{(+)}=O, & \left.E^{(+)}\right|_{x_{1}=0}=I_{2}, \\ \partial_{x_{1}} E^{(-)}-E^{(-)} \bar{L}\left(x_{1}, \xi_{n}\right)=O, & \left.E^{(-)}\right|_{x_{1}=0}=I_{2} .\end{cases}
$$

Let

$$
X^{( \pm)}=\left(\begin{array}{cc}
\exp \left(\mp \frac{1}{q+1} x_{1}^{q+1} \xi_{n}\right) & 0 \\
0 & \exp \left( \pm \frac{1}{q+1} x_{1}^{q+1} \xi_{n}\right)
\end{array}\right)
$$

We can proceed as follows:

(i) Neglecting the initial value for the moment, we have solutions $\tilde{E}^{(+, \theta)}=$ $F^{(+, \theta)} X^{(+)}$and $\tilde{E}^{(-, \theta)}=X^{(-)} F^{(-, \theta)}$ of the above equations on $\Xi^{\prime}(C, \theta)$. Here $F^{( \pm, \theta)}\left(x_{1} \xi_{n}\right)$ are infra-exponential functions of $\xi_{n}$, and we have $\boldsymbol{F}^{( \pm, \theta)} \boldsymbol{F}^{(\mp, \theta)}=\boldsymbol{I}_{2}$.

(ii) We next extend $F^{( \pm, \theta)}$ to $\Xi^{\prime \prime}(C)$. We can extend $F^{( \pm, \theta)}$ to a infraexponential function on this region.

(iii) We finally adjust the initial value. Let $\Xi(C, 0)=\Xi(C) \cap\left\{x_{1}=0\right\}$. $\Xi^{\prime \prime}(C)$ is not a usual conical neighborhood of $\Xi(C, 0)$, but it is a neighborhood of $\Xi(C, 0)$ in the topological sense. Therefore we can define $G^{( \pm, \theta)}=$ $\left.F^{(+, \theta)}\right|_{x_{1}=0}$ on $\Xi(C, 0)$. Then we have $G^{( \pm, \theta)} G^{(\mp, \theta)}=I_{2}$, and we define $H^{( \pm, \theta)}$ by

$$
\begin{aligned}
H^{(+, \theta)}\left(x_{1}, \xi_{n}\right) & =\tilde{E}^{(+, \theta)}\left(x_{1}, \xi_{n}\right)\left(G^{(+, \theta)}\left(\xi_{n}\right)\right)^{-1} \\
& =\tilde{E}^{(+, \theta)}\left(x_{1}, \xi_{n}\right) G^{(-, \theta)}\left(\xi_{n}\right) \\
& =F^{(+, \theta)}\left(x_{1}, \xi_{n}\right) X^{(+)}\left(x_{1}, \xi_{n}\right) G^{(-, \theta)}\left(\xi_{n}\right), \\
H^{(-, \theta)}\left(x_{1}, \xi_{n}\right) & =G^{(+, \theta)}\left(\xi_{n}\right) X^{(-)}\left(x_{1}, \xi_{n}\right) F^{(-, \theta)}\left(x_{1}, \xi_{n}\right) .
\end{aligned}
$$

This means that $H^{( \pm, \theta)}$ are the solutions of the above Cauchy problem, therefore we have $H^{( \pm, \theta)}=E^{( \pm)}$. After that, we can study these symbols on the whole $\Xi(C)$. 


\section{§4. Diagonalization of the Principal Symbol}

We assume (2), (3), (4), and (7). Let $C \gg 1$. We define

$$
\Omega^{o}(C)=\left\{\left(x, \xi^{\prime}\right) \in \Omega(C) ; x_{1} \neq 0\right\} .
$$

We will diagonalize $\bar{L}(x, \xi)$ in several steps. Let us consider the following Vandermonde's matrix on $\Omega^{\circ}(C)$ :

$$
\Delta\left(x, \xi^{\prime}\right)=\left(\begin{array}{cccc}
1 & 1 & \cdots & 1 \\
\varphi_{1}\left(x, \xi^{\prime}\right) & \varphi_{2}\left(x, \xi^{\prime}\right) & \cdots & \varphi_{m}\left(x, \xi^{\prime}\right) \\
\vdots & \vdots & & \vdots \\
\left(\varphi_{1}\left(x, \xi^{\prime}\right)\right)^{m-1} & \left(\varphi_{2}\left(x, \xi^{\prime}\right)\right)^{m-1} & \cdots & \left(\varphi_{m}\left(x, \xi^{\prime}\right)\right)^{m-1}
\end{array}\right) .
$$

It is easy to see that the $(\mu, v)$-component $\Delta_{(\mu, v)}^{-1}$ of the inverse matrix $\Delta^{-1}$ (in the sense of the usual multiplication, and not in the sense of the previous composition $\Delta \circ \Delta^{-1}$ ) satisfies

$$
\left|\Delta_{(\mu, \nu)}^{-1}\right| \leq \exists \text { constant }\left(\left|x_{1}\right|^{q_{\mu}} \operatorname{Im} \xi_{n}\right)^{-v+1} .
$$

Now let us calculate the inverse $\Delta^{-}\left(x, \xi^{\prime}\right)$ of $\Delta^{+}\left(x, \xi^{\prime}\right)=\Delta\left(x, \xi^{\prime}\right)$ in the sense of our composition this time. Let $f=\sum_{0 \leq j \leq i} f_{i, j}$ be a formal sum. We write $f=g$ or $f \underset{i, j}{g} g$ if $f_{i, j}=g_{i, j}$ for every $i$ and $j$. On the other hand, if $\sum_{j} f_{i, j}=$ $\sum_{j} g_{i, j}$ for every $i$, we write $f=g$. Note that $\sum_{j} f_{i, j}$ is a finite sum for each $i$. Finally we define $f \circ g=\sum_{i, j} h_{i, j}$ by

$$
h_{i, j}=\sum_{\substack{i^{\prime}+i^{\prime \prime}+\left|\alpha^{\prime}\right|=i \\ j^{\prime}+j^{\prime \prime}=j}} \frac{1}{\alpha^{\prime} !} \partial_{\xi^{\prime}}^{\alpha^{\prime}} f_{i^{\prime}, j^{\prime}} \partial_{x^{\prime}}^{\alpha^{\prime}} g_{i^{\prime \prime}, j^{\prime \prime}}
$$

Lemma 5. Let $C \gg a \gg 1$. We define $\Delta_{i, j}^{+}\left(x, \xi^{\prime}\right)=\delta_{i 0} \delta_{j 0} \Delta\left(x, \xi^{\prime}\right)$. Then there exist $\Delta_{i, j}^{-}\left(x, \xi^{\prime}\right) \in\left(\mathcal{O}\left(\Omega^{o}(C)\right)\right)^{m \times m}, 0 \leq j \leq i$, which satisfy $\Delta^{( \pm)}\left(x, \xi^{\prime}\right) \circ$ $\Delta^{(\mp)}\left(x, \xi^{\prime}\right)=I_{i}$, and we have

$$
\begin{gathered}
\left|\Delta_{i, j,(\mu, v)}^{+}\left(x, \xi^{\prime}\right)\right| \leq a\left(\operatorname{Im} \xi_{n}\right)^{\mu-1} \\
\left|\Delta_{i, j,(\mu, v)}^{-}\left(x, \xi^{\prime}\right)\right| \leq a^{2 i+1}(i-j) !\left|x_{1}\right|^{-q(m-1)(j+1)}\left(\operatorname{Im} \xi_{n}\right)^{-v+1-i}
\end{gathered}
$$

on $\Omega^{o}(C)$. Proof. Let $\Delta^{\prime}=\Delta \circ \Delta^{-1}$. In other words we define $\Delta_{i,(\mu, v)}^{\prime}=\sum_{\substack{1 \leq \kappa \leq m \\\left|\alpha^{\prime}\right|=i}} \frac{1}{\alpha^{\prime} !}$.
$\partial_{\xi^{\prime}}^{\alpha^{\prime}} \Delta \partial_{x^{\prime}}^{\alpha^{\prime}} \Delta^{-1}$. Then we have $\Delta_{0}^{\prime}=I_{m}$, and

$$
\left|\Delta_{i,(\mu, \nu)}^{\prime}\left(x, \xi^{\prime}\right)\right| \leq \exists a^{i+1} i !\left|x_{1}\right|^{q(1-v)}\left(\operatorname{Im} \xi_{n}\right)^{\mu-\nu-i} .
$$


We define

$$
\Delta_{i, j}^{\prime}= \begin{cases}\delta_{j 0} \Delta_{0}^{\prime}, & i=0 \\ \delta_{j 1} \Delta_{i}^{\prime}, & i \geq 1\end{cases}
$$

and $\Delta_{i, j}^{\prime \prime}=\delta_{i 0} \delta_{j 0} I_{m}-\sum_{\substack{i^{\prime}+i^{\prime \prime}+\left|\alpha^{\prime}\right|=i \\ i^{\prime} \neq 0 \\ j^{\prime}+j^{\prime \prime}=j}} \frac{1}{\alpha^{\prime} !} \partial_{\xi^{\prime}}^{\alpha^{\prime}} \Delta_{i^{\prime}, j^{\prime}}^{\prime} \partial_{x^{\prime}}^{\alpha^{\prime}} \Delta_{i^{\prime \prime}, j^{\prime \prime}}^{\prime \prime}$ by induction on $i . \quad$ By a direct calculation we can prove

$$
\left|\partial_{x^{\prime}}^{\alpha^{\prime}} \Delta_{i, j,(\mu, v)}^{\prime \prime}\left(x, \xi^{\prime}\right)\right| \leq \exists a^{2 i+\left|\alpha^{\prime}\right|+1}\left(i-j+\left|\alpha^{\prime}\right|\right) !\left|x_{1}\right|^{-q(m-1) j}\left(\operatorname{Im} \xi_{n}\right)^{\mu-v-i} .
$$

Defining $\Delta^{-}=\Delta^{-1} \circ \Delta^{\prime \prime}$ we obtain Lemma 5. Q.E.D.

Let us define $L^{\prime}(x, \xi)=\xi_{1} I_{m}+\bar{L}^{\prime}\left(x, \xi^{\prime}\right)$, where

$$
\bar{L}^{\prime}\left(x, \xi^{\prime}\right)=\Delta^{-}\left(x, \xi^{\prime}\right) \circ \bar{L}\left(x, \xi^{\prime}\right) \circ \Delta^{+}+\Delta^{-}\left(x, \xi^{\prime}\right) \circ \partial_{x_{1}} \Delta^{+} .
$$

Therefore we have $L(x, D) \Delta^{+}\left(x, D^{\prime}\right)=\Delta^{+}\left(x, D^{\prime}\right) \bar{L}(x, D)$ formally. Here we remind the reader that we have defined $\bar{L}=\sum_{i} \bar{L}_{i}=\sum_{0 \leq j \leq i} \bar{L}_{i, j}$, where $\bar{L}_{i, j}=$ $\delta_{j, 0} \bar{L}_{i}$. Therefore we have $\bar{L}^{\prime}=\sum_{0 \leq j \leq i} \bar{L}_{i, j}^{\prime}$ naturally. We have obtained the following

Lemma 6. If $C \gg a \gg 1$, then we have

$$
\begin{gathered}
\partial_{x_{1}} \Delta^{+}+\bar{L} \circ \Delta^{+}-\Delta^{+} \circ \bar{L}^{\prime}=O, \\
\left|\bar{L}_{0,(\mu, v)}^{\prime}+\delta_{\mu v} \varphi_{\mu}\left(x, \xi^{\prime}\right)\right| \leq a\left|x_{1}\right|^{-q(m-1)-1}, \\
\left|\bar{L}_{i, j,(\mu, v)}^{\prime}\right| \leq a^{i+1}(i-j) !\left|x_{1}\right|^{-q(m-1)(j+1)}\left(\operatorname{Im} \xi_{n}\right)^{-i+1}, \quad i \geq 1
\end{gathered}
$$

on $\Omega^{o}$.

\section{§5. Miscellanea}

\section{§5.1. Formal Norms}

The next step is the most important part, and will be discussed in the next section. Here we give some preliminaries. At first we define formal norms similar to [3]. Let $\omega \subset \mathbf{C}^{n} \times \mathbf{C}^{n-1}$ be an open set. Let $f=\sum_{j} f_{j}\left(x, \xi^{\prime}\right)$ be a formal sum where $f_{j}\left(x, \xi^{\prime}\right) \in \mathcal{O}(\omega)$. We define $N(f, \omega)=N\left(f, \omega, t, x, \xi^{\prime}\right)$ by

$$
N(f, \omega)=\sum_{\substack{j \in \mathbf{Z}_{+} \\ \alpha^{\prime}, \beta^{\prime} \in \mathbf{Z}_{+}^{n-1}}} \frac{2(2 n)^{-j} j !}{\left(j+\left|\alpha^{\prime}\right|\right) !\left(j+\left|\beta^{\prime}\right|\right) !}\left|\partial_{x^{\prime}}^{\alpha^{\prime}} \partial_{\xi^{\prime}}^{\beta^{\prime}} f_{j}\left(x, \xi^{\prime}\right)\right|\left|\xi^{\prime}\right|^{j+\left|\beta^{\prime}\right|} t^{2 j+\left|\alpha^{\prime}+\beta^{\prime}\right|},
$$


where $\left(x, \xi^{\prime}\right) \in \omega$. This is a formal series in $t$. In place of $\left|\partial_{x^{\prime}}^{\alpha^{\prime}} \partial_{\xi^{\prime}}^{\beta^{\prime}} f_{j}\left(x, \xi^{\prime}\right)\right|$. $\left|\xi^{\prime}\right|^{j+\left|\beta^{\prime}\right|}$ in the above definition, [3] considered its supremum. This means that our formal norm (resp. the formal norm of [3]) is a formal series in $t$, whose coefficients are continuous functions on $\omega$ (resp. constants). Let $f\left(x, D^{\prime}\right) \in$ $\mathscr{E}^{\mathbf{R}}(\omega)$. Let $\varepsilon>0$ be an arbitrary number. Shrinking $\omega$ if necessary, we can choose its formal symbol $f\left(x, \xi^{\prime}\right)$ such that $N(f, \omega)$ is convergent and $N(f, \omega) \leq \exists C_{\varepsilon} \exp \left(\varepsilon \operatorname{Im} \xi_{n}\right)$ for $0<t \ll 1,\left(x, \xi^{\prime}\right) \in \omega$. Conversely, such a formal series defines a holomorphic microlocal operator on $\omega$.

If $f=\sum_{j} f_{j}\left(x, \xi^{\prime}\right)$ satisfies $f_{j}=0$ for $0 \leq j \leq j_{0}-1$, then we define $N_{j_{0}}(f, \omega)=N_{j_{0}}\left(f, \omega, t, x, \xi^{\prime}\right)$ by

$$
N_{j_{0}}(f, \omega)=\sum_{\substack{j \in \mathbf{Z}_{+} \\ \alpha^{\prime}, \beta^{\prime} \in \mathbf{Z}_{+}^{n-1}}} \frac{2(2 n)^{-j} j !}{\left(j+\left|\alpha^{\prime}\right|\right) !\left(j+\left|\beta^{\prime}\right|\right) !}\left|\partial_{x^{\prime}}^{\alpha^{\prime}} \partial_{\xi^{\prime}}^{\beta^{\prime}} f_{j+j_{0}}\left(x, \xi^{\prime}\right)\right|\left|\xi^{\prime}\right|^{j+\left|\beta^{\prime}\right|} t^{2 j+\left|\alpha^{\prime}+\beta^{\prime}\right|} .
$$

If $f=\sum f_{j}\left(x, \xi^{\prime}\right)$ and $h_{0}\left(x, \xi^{\prime}\right) \in \mathcal{O}(\omega)$, then we define $h_{0} f=\sum h_{0} f_{j}$. If $a=$ $\sum a_{j} t^{j}$ and $b=\sum b_{j} t^{j}\left(a_{j}, b_{j} \in \mathbf{R}\right)$ satisfy $a_{j} \leq b_{j}$ for every $j$, then we write $a \lesssim b$. As in [3] we have the following

Lemma 7. If $f=\sum_{j \geq 0} f_{j}, g=\sum_{j \geq 0} g_{j}$, and $h_{0} \in \mathcal{O}(\omega)$, then we have

$$
N(f \circ g, \omega) \lesssim N(f, \omega) N(g, \omega), \quad N\left(h_{0} f, \omega\right) \lesssim N(f, \omega) N\left(h_{0}, \omega\right)
$$

on $\omega$. If $f=\sum_{j \geq j_{0}} f_{j}, g=\sum_{j \geq k_{0}} g_{j}$, and $h_{0} \in \mathcal{O}(\omega)$, then we have

$$
N_{j_{0}+k_{0}}(f \circ g, \omega) \lesssim N_{j_{0}}(f, \omega) N_{k_{0}}(g, \omega), \quad N_{j_{0}}\left(h_{0} f, \omega\right) \lesssim N_{j_{0}}(f, \omega) N\left(h_{0}, \omega\right)
$$

on $\omega$.

We finally consider a formal series $f=\sum_{0 \leq j \leq i} f_{i, j}\left(x, \xi^{\prime}\right)$, and define $f^{j}=$ $\sum_{i} f_{i, j}$ for each $j$ (On the other hand, we define $f_{i}=\sum_{j} f_{i, j}$ for each $i$ ). For such a formal series with double indices, we define

$$
\mathcal{N}(f, \omega)=\mathcal{N}\left(f, \omega, t_{1}, t_{2}, x, \xi^{\prime}\right)=\sum_{j} N_{j}\left(f^{j}, \omega, t_{1}, x, \xi^{\prime}\right) t_{2}^{j}
$$

If $f=\sum_{0 \leq j \leq i} f_{i, j}\left(x, \xi^{\prime}\right)$ and $g=\sum_{0 \leq j \leq i} g_{i, j}\left(x, \xi^{\prime}\right)$, then we define $f \circ g=\sum h_{i, j}$, where $h_{i, j}$ is defined by

$$
h_{i, j}=\sum_{\substack{i^{\prime}+i^{\prime \prime}+\left|\alpha^{\prime}\right|=i \\ j^{\prime}+j^{\prime \prime}=j}} \frac{1}{\alpha^{\prime !}} \partial_{\xi^{\prime}}^{\alpha^{\prime}} f_{i^{\prime}, j^{\prime}} \partial_{x^{\prime}}^{\alpha^{\prime}} g_{i^{\prime \prime}, j^{\prime \prime}}
$$


as before, and if $h_{0,0}\left(x, \xi^{\prime}\right) \in \mathcal{O}(\omega)$ then we define $h_{0,0} f=\sum_{0 \leq j \leq i} h_{0,0} f_{i, j}$. If $a=$ $\sum a_{l, j} t_{1}^{i} t_{2}^{j}$ and $b=\sum b_{l, j} t_{1}^{i} t_{2}^{j}\left(a_{i, j}, b_{i, j} \in \mathbf{R}\right)$ satisfy $a_{i, j} \leq b_{i, j}$ for every $i$ and $j$, then we write $a \lesssim b$. It is easy to see the following

Lemma 8. If $f=\sum_{0 \leq j \leq i} f_{i, j}\left(x, \xi^{\prime}\right), \quad g=\sum_{0 \leq j \leq i} g_{i, j}\left(x, \xi^{\prime}\right)$, and $h_{0,0}\left(x, \xi^{\prime}\right) \in$ $\mathcal{O}(\omega)$, then we have

$$
\mathscr{N}(f \circ g, \omega) \lesssim \mathscr{N}(f, \omega) \mathscr{N}(g, \omega), \quad \mathcal{N}\left(h_{0,0} f, \omega\right) \lesssim \mathscr{N}(f, \omega) \mathscr{N}\left(h_{0,0}, \omega\right)
$$

on $\omega$.

\section{§5.2. Phase Functions}

We next give a geometric discussion.

Lemma 9. Let $\theta \in[0,2 \pi]$ be an arbitrary number. We can choose some numbers $\theta_{k}^{\prime}, \theta_{k}^{\prime \prime}(1 \leq k \leq m)$ satisfying the following conditions:

(i) $\theta \in\left(\theta_{m}^{\prime}, \theta_{m}^{\prime \prime}\right) \subset\left(\theta_{m-1}^{\prime}, \theta_{m-1}^{\prime \prime}\right) \subset \cdots \subset\left(\theta_{1}^{\prime}, \theta_{1}^{\prime \prime}\right)$,

(ii) $\left(q_{i}+1\right) \theta_{i}^{\prime}+\arg a_{i}\left(x^{*}\right) \notin \frac{\pi}{2} \mathbf{Z}_{+}, \quad \theta_{i}^{\prime \prime}-\theta_{i}^{\prime}=\pi /\left(q_{i}+1\right)$,

(iii) If $q_{i}=q_{j}, i \neq j$, then we have

$$
\left(q_{i}+1\right) \theta_{i}^{\prime}+\arg \left(a_{i}\left(x^{*}\right)-a_{j}\left(x^{*}\right)\right) \notin \frac{\pi}{2} \mathbf{Z}_{+}, \quad \theta_{i}^{\prime}=\theta_{j}^{\prime}, \quad \theta_{i}^{\prime \prime}=\theta_{j}^{\prime \prime} .
$$

Proof. We first remind the reader that $q_{1} \leq q_{2} \leq \cdots \leq q_{m}$. We can define $\theta_{1}^{\prime}$ such that

$$
\begin{gathered}
\left(q_{j}+1\right) \theta_{1}^{\prime}+\arg a_{j}\left(x^{*}\right) \notin \frac{\pi}{2} \mathbf{Z}_{+}, \quad \text { if } q_{1}=q_{j}, \\
\left(q_{j}+1\right) \theta_{1}^{\prime}+\arg \left(a_{j}\left(x^{*}\right)-a_{k}\left(x^{*}\right)\right) \notin \frac{\pi}{2} \mathbf{Z}_{+}, \quad \text { if } q_{1}=q_{j}=q_{k}, j \neq k, \\
\theta \in\left(\theta_{1}^{\prime}, \theta_{1}^{\prime}+\pi /\left(q_{1}+1\right)\right),
\end{gathered}
$$

and $\theta_{1}^{\prime \prime}=\theta_{1}^{\prime}+\pi /\left(q_{1}+1\right)$. Assume that $2 \leq i \leq m$ and that we have already chosen $\theta_{k}^{\prime}$ satisfying $\theta_{k}^{\prime}<\theta<\theta_{k}^{\prime}+\pi /\left(q_{k}+1\right)$ for $1 \leq k \leq i-1$, and let us define $\theta_{i}^{\prime}$. In case of $q_{i}=q_{i-1}$, we define $\theta_{i}^{\prime}=\theta_{i-1}^{\prime}$. In case of $q_{i}>q_{i-1}$ then we choose $\theta_{i}^{\prime}$ satisfying

$$
\left(q_{i}+1\right) \theta_{i}^{\prime}+\arg a_{J}\left(x^{*}\right) \notin \frac{\pi}{2} \mathbf{Z}_{+}, \quad \text { if } q_{i}=q_{j},
$$




$$
\begin{gathered}
\left(q_{i}+1\right) \theta_{i}^{\prime}+\arg \left(a_{j}\left(x^{*}\right)-a_{k}\left(x^{*}\right)\right) \notin \frac{\pi}{2} \mathbb{Z}_{+}, \quad \text { if } q_{i}=q_{j}=q_{k}, j \neq k, \\
\theta \in\left(\theta_{i}^{\prime}, \theta_{i}^{\prime}+\pi /\left(q_{i}+1\right)\right) \subset\left(\theta_{i-1}^{\prime}, \theta_{i-1}^{\prime}+\pi /\left(q_{i-1}+1\right)\right) .
\end{gathered}
$$

Since $\pi /\left(q_{i}+1\right)<\pi /\left(q_{i-1}+1\right)$, we can certainly choose such a $\theta_{i}^{\prime}$. In both cases we define $\theta_{i}^{\prime \prime}=\theta_{i}^{\prime}+\pi /\left(q_{i}+1\right)$. It is easy to see (i)-(iii). Q.E.D.

We define $\theta_{i}^{\prime \prime \prime}=\left(\theta_{i}^{\prime}+\theta_{i}^{\prime \prime}\right) / 2$, and formally let $q_{0}=q_{1}, \theta_{0}^{\prime}=\theta_{1}^{\prime}, \theta_{0}^{\prime \prime}=\theta_{1}^{\prime \prime}$, $\theta_{0}^{\prime \prime \prime}=\theta_{1}^{\prime \prime \prime}$. We define $x_{1}(\lambda)=\left(e^{-\sqrt{-1} \theta_{\lambda}^{\prime \prime \prime}} x_{1}\right)^{q_{\lambda}+1}$. Let $1 \ll a \ll C$. We define

$$
\begin{aligned}
\tilde{\Omega}_{\lambda}^{\prime}(C)=\{ & \left(x, \xi^{\prime}\right) \in \mathbb{C}^{n} \times \mathbb{C}^{n-1} ; C^{1 / 10}\left|x^{\prime}\right|<1, \\
& C^{1 / 10}\left|\xi^{\prime \prime \prime}\right|<\operatorname{Im} \xi_{n}, C^{1 / 10}\left|\operatorname{Re} \xi_{n}\right|<\operatorname{Im} \xi_{n}, \\
& 2^{-1} C^{4}\left(\operatorname{Im} \xi_{n}\right)^{-\left(q_{\lambda}+1\right) /(m q+m)}<\operatorname{Re}\left(x_{1}(\lambda)\right)+a^{-1}\left|\operatorname{Im}\left(x_{1}(\lambda)\right)\right|<C^{-q_{\lambda}-1}, \\
& \left.\theta_{\lambda}^{\prime}<\arg x_{1}<\theta_{\lambda}^{\prime \prime}\right\} \\
\Omega_{\lambda}^{\prime}(C)=\{ & \left(x, \xi^{\prime}\right) \in \mathbb{C}^{n} \times \mathbb{C}^{n-1} ; C\left|x^{\prime}\right|<1, C\left|\xi^{\prime \prime \prime}\right|<\operatorname{Im} \xi_{n}, C\left|\operatorname{Re} \xi_{n}\right|<\operatorname{Im} \xi_{n}, \\
& C^{4}\left(\operatorname{Im} \xi_{n}\right)^{-\left(q_{\lambda}+1\right) /(m q+m)}<\operatorname{Re}\left(x_{1}(\lambda)\right)+a^{-1}\left|\operatorname{Im}\left(x_{1}(\lambda)\right)\right|<C^{-q_{\lambda}-1}, \\
& \left.\theta_{\lambda}^{\prime}<\arg x_{1}<\theta_{\lambda}^{\prime \prime}\right\}
\end{aligned}
$$

(See the figure below). We have the following

Lemma 10. (i) If $C<C^{\prime}$, then we have

$$
\begin{array}{ccc}
\tilde{\Omega}_{\lambda}^{\prime}(C) & \supset & \tilde{\Omega}_{\lambda}^{\prime}\left(C^{\prime}\right) \\
\cup & \cup \\
\Omega_{\lambda}^{\prime}(C) & \supset & \Omega_{\lambda}^{\prime}\left(C^{\prime}\right) .
\end{array}
$$

(ii) If $\left(x, \xi^{\prime}\right) \in \Omega_{\lambda}^{\prime}(C),\left(y, \eta^{\prime}\right) \in \mathbb{C}^{n} \times \mathbb{C}^{n-1}$, and $\left|y_{j}\right|<C^{-1 / 5},\left|\eta_{j}^{\prime}\right| / \operatorname{Im} \xi_{n}<$ $C^{-1 / 5}$ for $2 \leq j \leq n$, then we have $\left(x_{1}, x^{\prime}+y^{\prime}, \xi^{\prime}+\eta^{\prime}\right) \in \tilde{\Omega}_{\lambda}^{\prime}(C)$.

(iii) If $\left(x, \xi^{\prime}\right) \in \Omega_{\lambda}^{\prime}\left(C^{2}\right),\left(y, \eta^{\prime}\right) \in \mathbb{C}^{n} \times \mathbb{C}^{n-1}$, and $\left|y_{j}\right|<C^{-2},\left|\eta_{j}^{\prime}\right| / \operatorname{Im} \xi_{n}<$ $C^{-2}$ for $2 \leq j \leq n$, then we have $\left(x_{1}, x^{\prime}+y^{\prime}, \xi^{\prime}+\eta^{\prime}\right) \in \Omega_{\lambda}^{\prime}(C)$.

(iv) Let $1 \leq \lambda<\mu \leq m$. We define $t_{\lambda, \mu}^{ \pm} \in \mathbb{C}$ as follows. If $q_{\lambda}=q_{\mu}$, then $t_{\lambda, \mu}^{ \pm} \in \mathbb{C}$ is the point at which $\pm \operatorname{Re}\left(\left(a_{\mu}\left(x^{*}\right)-a_{\lambda}\left(x^{*}\right)\right) x_{1}^{q_{\lambda}+1}\right)$ takes its maximum when $\left(x, \xi^{\prime}\right)$ belongs to the closure of $\tilde{\Omega}_{\lambda}^{\prime}(C)$. If $q_{\lambda} \neq q_{\mu}$, then it is the point at which $\mp \operatorname{Re}\left(a_{\lambda}\left(x^{*}\right) z^{q_{\lambda}+1}\right)$ takes its maximum. Then for any $\lambda$ and $\mu,\left(t_{\lambda, \mu}^{+}\right)^{q_{\lambda}+1}$ is one of $\left\{A_{\lambda}^{\prime}, A_{\lambda}^{\prime \prime}\right\}$, and $\left(t_{\lambda, \mu}^{-}\right)^{q_{\lambda}+1}$ is the other. Here $A_{\lambda}^{\prime}, A_{\lambda}^{\prime \prime}, A_{\lambda}^{\prime \prime \prime}$ are the points indicated in the following figure. 


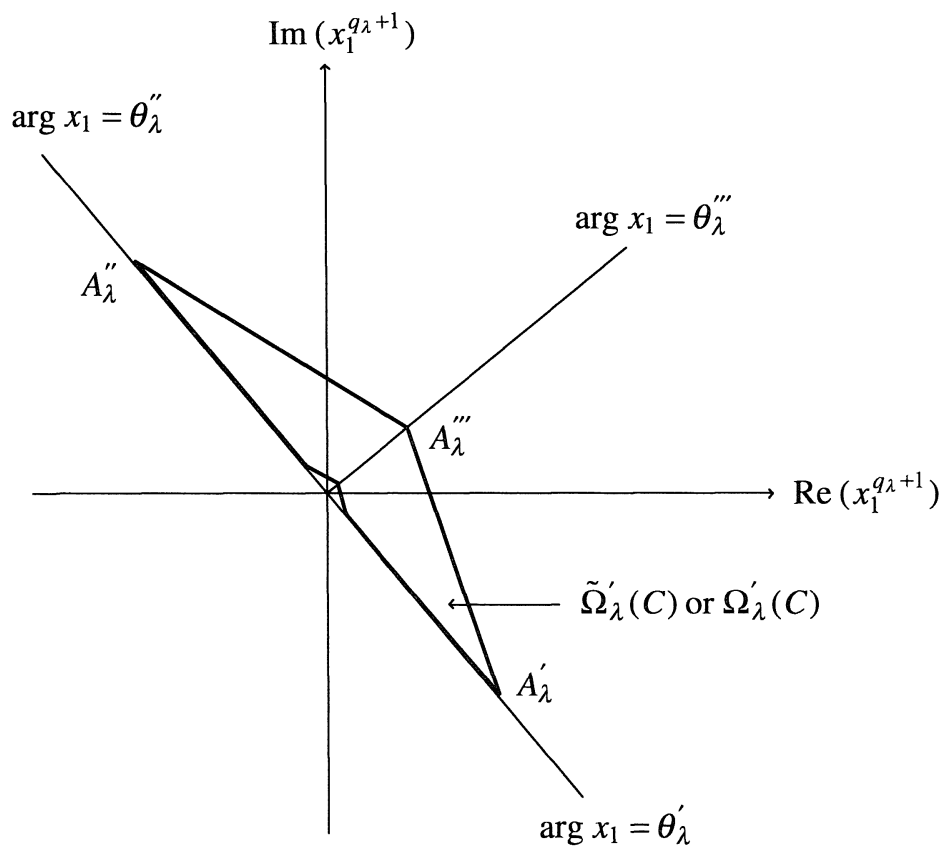

Proof. (i)-(iii) are direct consequences of a simple calculation, and we prove (iv). We first consider the case $q_{\lambda}=q_{\mu}$. From (iii) of Lemma 9 it follows that in the above figure the line segment combining $\left(a_{\mu}\left(x^{*}\right)-a_{\lambda}\left(x^{*}\right)\right) A_{\lambda}^{\prime}$ and $\left(a_{\mu}\left(x^{*}\right)-a_{\lambda}\left(x^{*}\right)\right) A_{\lambda}^{\prime \prime}$ is parallel with neither the real axis nor the imaginary axis. Since $a>0$ is large enough (compared with the slopes of these line segments), we may assume that we have either

$$
\begin{aligned}
\operatorname{Re}\left(\left(a_{\mu}\left(x^{*}\right)-a_{\lambda}\left(x^{*}\right)\right) A_{\lambda}^{\prime}\right) & <\operatorname{Re}\left(\left(a_{\mu}\left(x^{*}\right)-a_{\lambda}\left(x^{*}\right)\right) A_{\lambda}^{\prime \prime \prime}\right) \\
& <\operatorname{Re}\left(\left(a_{\mu}\left(x^{*}\right)-a_{\lambda}\left(x^{*}\right)\right) A_{\lambda}^{\prime \prime}\right)
\end{aligned}
$$

or

$$
\begin{aligned}
\operatorname{Re}\left(\left(a_{\mu}\left(x^{*}\right)-a_{\lambda}\left(x^{*}\right)\right) A_{\lambda}^{\prime}\right) & >\operatorname{Re}\left(\left(a_{\mu}\left(x^{*}\right)-a_{\lambda}\left(x^{*}\right)\right) A_{\lambda}^{\prime \prime \prime}\right) \\
& >\operatorname{Re}\left(\left(a_{\mu}\left(x^{*}\right)-a_{\lambda}\left(x^{*}\right)\right) A_{\lambda}^{\prime \prime}\right) .
\end{aligned}
$$

Accordingly, if $\left(x, \xi^{\prime}\right)$ belongs to the closure of $\tilde{\Omega}_{\lambda}^{\prime}(C)$, we have either

$$
\begin{aligned}
\operatorname{Re}\left(\left(a_{\mu}\left(x^{*}\right)-a_{\lambda}\left(x^{*}\right)\right) A_{\lambda}^{\prime}\right) & \leq \operatorname{Re}\left(\left(a_{\mu}\left(x^{*}\right)-a_{\lambda}\left(x^{*}\right)\right) x_{1}^{q_{\lambda}+1}\right) \\
& \leq \operatorname{Re}\left(\left(a_{\mu}\left(x^{*}\right)-a_{\lambda}\left(x^{*}\right)\right) A_{\lambda}^{\prime \prime}\right)
\end{aligned}
$$


or

$$
\begin{aligned}
\operatorname{Re}\left(\left(a_{\mu}\left(x^{*}\right)-a_{\lambda}\left(x^{*}\right)\right) A_{\lambda}^{\prime}\right) & \geq \operatorname{Re}\left(\left(a_{\mu}\left(x^{*}\right)-a_{\lambda}\left(x^{*}\right)\right) x_{1}^{q_{\lambda}+1}\right) \\
& \geq \operatorname{Re}\left(\left(a_{\mu}\left(x^{*}\right)-a_{\lambda}\left(x^{*}\right)\right) A_{\lambda}^{\prime \prime}\right) .
\end{aligned}
$$

This proves (iv) for the case $q_{\lambda}=q_{\mu}$. We can prove the case $q_{\lambda} \neq q_{\mu}$ similarly. Q.E.D.

Let $1 \leq \lambda<\mu \leq m$. If $\left(x, \xi^{\prime}\right) \in \tilde{\Omega}_{\lambda}^{\prime}$, then we define a continuous curve $\gamma_{\lambda, \mu}^{ \pm}\left(x_{1}\right)$ from $\left(t_{\lambda, \mu}^{ \pm}\right)^{q_{\lambda}+1}$ to $x_{1}^{q_{\lambda}+1}$ as follows. Let us consider the case $t_{\lambda, \mu}^{ \pm}=A_{\lambda}^{\prime}$ (resp. $\left.t_{\lambda, \mu}^{ \pm}=A_{\lambda}^{\prime \prime}\right)$. If $\arg x_{1} \leq \theta_{\lambda}^{\prime \prime \prime}$ (resp. $\left.\arg x_{1} \geq \theta_{\lambda}^{\prime \prime \prime}\right)$, then $\gamma_{\lambda, \mu}^{ \pm}\left(x_{1}\right)$ is a line segment from $\left(t_{\lambda, \mu}^{+}\right)^{q_{\lambda}+1}$ to $x_{1}^{q_{\lambda}+1}$. If $\arg x_{1}>\theta_{\lambda}^{\prime \prime \prime}\left(\right.$ resp. $\left.\arg x_{1}<\theta_{\lambda}^{\prime \prime \prime}\right)$, then $\gamma_{\lambda, \mu}^{ \pm}\left(x_{1}\right)$ is the union of two line segments: one from $\left(t_{\lambda, \mu}^{ \pm}\right)^{q_{\lambda}+1}$ to $y_{1}^{q_{\lambda}+1}$, and the other from $y_{1}^{q_{\lambda}+1}$ to $x_{1}^{q_{\lambda}+1}$. Here $y_{1}$ is the point defined by $\operatorname{Re}\left(y_{1}(\lambda)\right)+$ $a^{-1}\left|\operatorname{Im} y_{1}(\lambda)\right|=\operatorname{Re}\left(x_{1}(\lambda)\right)+a^{-1}\left|\operatorname{Im} x_{1}(\lambda)\right|, \quad$ arg $y_{1}=\theta_{\lambda}^{\prime \prime \prime}$. Finally we define $\delta_{\lambda, \mu}^{ \pm}\left(x_{1}\right)=\left\{t \in \mathbf{C} ; t^{q_{\lambda}+1} \in \gamma_{\lambda, \mu}^{ \pm}\left(x_{1}\right)\right\}$, and denote by $\rho_{\lambda, \mu}^{ \pm}\left(x_{1}\right)$ the length of $\delta_{\lambda, \mu}^{ \pm}\left(x_{1}\right)$.

Note that we have

$$
\begin{aligned}
& \left(x, \xi^{\prime}\right) \in \tilde{\Omega}_{\lambda}^{\prime}(C), t \in \delta_{\lambda, \mu}^{ \pm}\left(x_{1}\right) \backslash\left\{t_{\lambda, \mu}^{ \pm}\right\} \Rightarrow\left(t, x^{\prime}, \xi^{\prime}\right) \in \tilde{\Omega}_{\lambda}^{\prime}(C), \\
& \left(x, \xi^{\prime}\right) \in \Omega_{\lambda}^{\prime}(C), t \in \delta_{\lambda, \mu}^{ \pm}\left(x_{1}\right) \backslash\left\{t_{\lambda, \mu}^{ \pm}\right\} \Rightarrow\left(t, x^{\prime}, \xi^{\prime}\right) \in \Omega_{\lambda}^{\prime}(C) .
\end{aligned}
$$

We illustrate the case $t_{\lambda, \mu}^{ \pm}=A_{\lambda}^{\prime \prime}, \arg x_{1} \leq \theta_{\lambda}^{\prime \prime \prime}$ in the following figure.

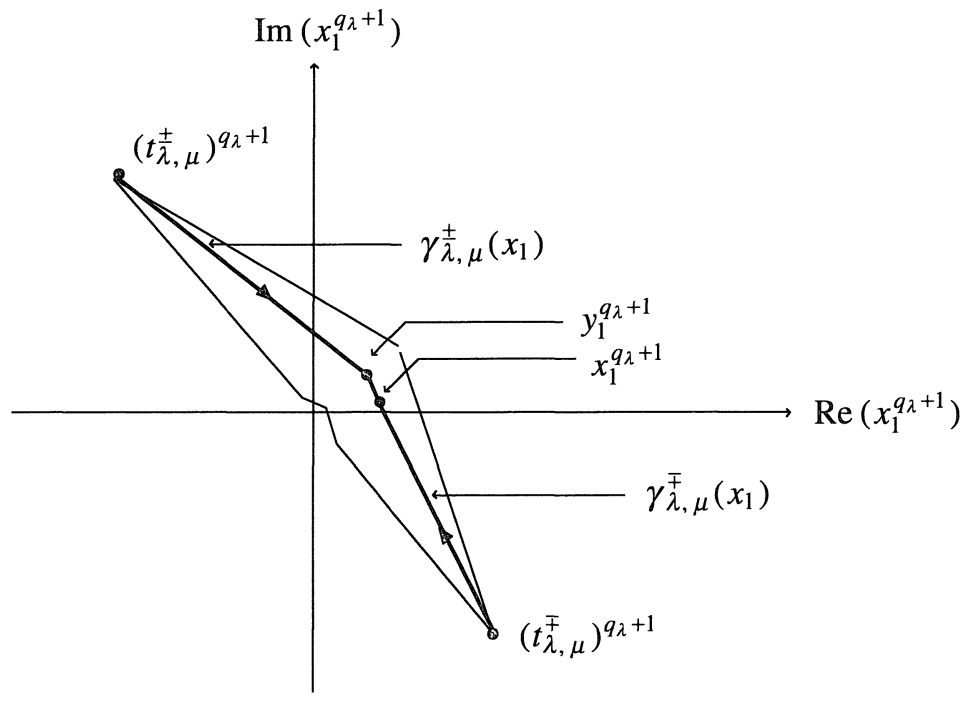


Let $1 \ll a \ll C_{0} \ll C_{1} \ll \cdots \ll C_{m}$, and let $\tilde{\Omega}_{\lambda}^{\prime}=\tilde{\Omega}_{\lambda}^{\prime}\left(C_{\lambda}\right), \quad \Omega_{\lambda}^{\prime}=\Omega_{\lambda}^{\prime}\left(C_{\lambda}\right)$. Therefore we have

$$
\begin{array}{cccc}
\tilde{\Omega}_{0}^{\prime} \supset \cdots & \tilde{\Omega}_{m}^{\prime} \\
\cup & & & \cup \\
\Omega_{0}^{\prime} \supset & \cdots \supset{ }_{m}^{\prime} .
\end{array}
$$

The next lemma will be important in the next section.

Lemma 11. Let $1 \leq \lambda<\mu \leq m, \quad\left(x, \xi^{\prime}\right) \in \tilde{\Omega}_{\lambda}^{\prime}, \quad t \in \delta_{\bar{\lambda}, \mu}^{ \pm}\left(x_{1}\right)$, and let $\varphi_{\lambda, \mu}\left(x, t, \xi^{\prime}\right)=-\int_{t}^{x_{1}}\left(\bar{L}_{0,(\lambda, \lambda)}^{\prime}\left(s, x^{\prime}, \xi^{\prime}\right)-\bar{L}_{0,(\mu, \mu)}^{\prime}\left(s, x^{\prime}, \xi^{\prime}\right)\right) d s$. Then we have

$$
\pm \operatorname{Re} \varphi_{\lambda, \mu}\left(x, t, \xi^{\prime}\right) \leq-a^{-4}\left|t^{q_{\lambda}+1}-x_{1}^{q_{\lambda}+1}\right| \operatorname{Im} \xi_{n}
$$

Proof. Let

$$
\theta_{0}= \begin{cases}\arg \left(a_{\mu}\left(x^{*}\right)-a_{\lambda}\left(x^{*}\right)\right), & q_{\mu}=q_{\lambda} \\ \arg \left(-a_{\lambda}\left(x^{*}\right)\right), & q_{\mu} \neq q_{\lambda}\end{cases}
$$

We denote

$$
\begin{aligned}
& \bar{L}_{0,(v, v)}^{\prime}\left(x, \xi^{\prime}\right)=x_{1}^{q_{v}} a_{v}\left(x, \xi^{\prime}\right)+b_{v}\left(x, \xi^{\prime}\right), \\
& a_{v}\left(x, \xi^{\prime}\right)=a_{v}\left(0, x^{\prime}, \xi^{\prime}\right)+x_{1}^{1 / m^{\prime}} a_{v}^{\prime}\left(x, \xi^{\prime}\right) .
\end{aligned}
$$

By Lemma 6 we may assume $\left|a_{v}^{\prime}\left(x, \xi^{\prime}\right)\right| \leq a \operatorname{Im} \xi_{n},\left|b_{v}\left(x, \xi^{\prime}\right)\right| \leq a\left|x_{1}\right|^{-q(m-1)-1}$. We have $\pm \operatorname{Re} \varphi_{\lambda, \mu}\left(x, t, \xi^{\prime}\right)=\mathrm{I}+\mathrm{II}+\mathrm{III}+\mathrm{IV}$, where

$$
\begin{aligned}
\mathrm{I}= & \mp \operatorname{Re}\left(e^{-\sqrt{-1} \theta_{0}} a_{\lambda}\left(0, x^{\prime}, \xi^{\prime}\right)\right) \operatorname{Re}\left(e^{\sqrt{-1} \theta_{0}}\left(x_{1}^{q_{\lambda}+1}-t^{q_{\lambda}+1}\right)\right) /\left(q_{\lambda}+1\right) \\
& \pm \operatorname{Re}\left(e^{-\sqrt{-1} \theta_{0}} a_{\mu}\left(0, x^{\prime}, \xi^{\prime}\right)\right) \operatorname{Re}\left(e^{\sqrt{-1} \theta_{0}}\left(x_{1}^{q_{\mu}+1}-t^{q_{\mu}+1}\right)\right) /\left(q_{\mu}+1\right), \\
\mathrm{II}= & \pm \operatorname{Im}\left(e^{-\sqrt{-1} \theta_{0}} a_{\lambda}\left(0, x^{\prime}, \xi^{\prime}\right)\right) \operatorname{Im}\left(e^{\sqrt{-1} \theta_{0}}\left(x_{1}^{q_{\lambda}+1}-t^{q_{\lambda}+1}\right)\right) /\left(q_{\lambda}+1\right) \\
& \mp \operatorname{Im}\left(e^{-\sqrt{-1} \theta_{0}} a_{\mu}\left(0, x^{\prime}, \xi^{\prime}\right)\right) \operatorname{Im}\left(e^{\sqrt{-1} \theta_{0}}\left(x_{1}^{q_{\mu}+1}-t^{q_{\mu}+1}\right)\right) /\left(q_{\mu}+1\right), \\
\mathrm{III}= & \mp \operatorname{Re}\left(\int_{t}^{x_{1}}\left(s^{q_{\lambda}+\left(1 / m^{\prime}\right)} a_{\lambda}^{\prime}\left(s, x^{\prime}, \xi^{\prime}\right)-s^{q_{\mu}+\left(1 / m^{\prime}\right)} a_{\mu}^{\prime}\left(s, x^{\prime}, \xi^{\prime}\right)\right) d s\right), \\
\mathrm{IV}= & \mp \operatorname{Re}\left(\int_{t}^{x_{1}}\left(b_{\lambda}\left(s, x^{\prime}, \xi^{\prime}\right)-b_{\mu}\left(s, x^{\prime}, \xi^{\prime}\right)\right) d s\right) .
\end{aligned}
$$

We can prove

$$
\mathrm{I} \leq-\frac{1}{2 a^{3}\left(q_{\lambda}+1\right)}\left|x_{1}^{q_{\lambda}+1}-t^{q_{\lambda}+1}\right| \operatorname{Im} \xi_{n}
$$


as follows. We first consider the case $q_{\lambda}=q_{\mu}$. Since we have

$$
\left|\arg \left(e^{-\sqrt{-1} \theta_{0}}\left(a_{\mu}\left(0, x^{\prime}, \xi^{\prime}\right)-a_{\lambda}\left(0, x^{\prime}, \xi^{\prime}\right)\right)\right)\right|<a^{-10}
$$

it follows that $\operatorname{Re}\left(e^{-\sqrt{-1} \theta_{0}}\left(a_{\mu}\left(0, x^{\prime}, \xi^{\prime}\right)-a_{\lambda}\left(0, x^{\prime}, \xi^{\prime}\right)\right)\right) \geq a^{-1 / 2} \operatorname{Im} \xi_{n}$. From Lemma 10 it follows that $\pm \operatorname{Re}\left(e^{\sqrt{-1} \theta_{0}}\left(x_{1}^{q_{\lambda}+1}-t^{q_{\lambda}+1}\right)\right) \leq-a^{-5 / 2}\left|x_{1}^{q_{\lambda}+1}-t^{q_{\lambda}+1}\right|$. Therefore we obtain

$$
\begin{aligned}
\mathbf{I} & = \pm \operatorname{Re}\left(e^{-\sqrt{-1} \theta_{0}}\left(a_{\mu}\left(0, x^{\prime}, \xi^{\prime}\right)-a_{\lambda}\left(0, x^{\prime}, \xi^{\prime}\right)\right)\right) \operatorname{Re}\left(e^{\sqrt{-1} \theta_{0}}\left(x_{1}^{q_{\lambda}+1}-t^{q_{\lambda}+1}\right)\right) /\left(q_{\lambda}+1\right) \\
& \leq-\frac{1}{a^{3}\left(q_{\lambda}+1\right)}\left|x_{1}^{q_{\lambda}+1}-t^{q_{\lambda}+1}\right| \operatorname{Im} \xi_{n} .
\end{aligned}
$$

We next consider the case $q_{\lambda} \neq q_{\mu}$. We can similarly prove

$$
\begin{gathered}
\pm \operatorname{Re}\left(e^{-\sqrt{-1} \theta_{0}} a_{\lambda}\left(0, x^{\prime}, \xi^{\prime}\right)\right) \operatorname{Re}\left(e^{\sqrt{-1} \theta_{0}}\left(x_{1}^{q_{\lambda}+1}-t^{q_{\lambda}+1}\right)\right) /\left(q_{\lambda}+1\right) \\
\geq \frac{1}{a^{3}\left(q_{\lambda}+1\right)}\left|x_{1}^{q_{\lambda}+1}-t^{q_{\lambda}+1}\right| \operatorname{Im} \xi_{n}
\end{gathered}
$$

If $\ell \in \mathbb{Z}_{+} / m^{\prime}$ satisfies $0 \leq \ell \leq\left(m^{\prime}-1\right) / m^{\prime}$, then we have

$$
\arg \left(x_{1}^{\ell} t^{q_{\lambda}-\ell+\left(\left(m^{\prime}-1\right) / m^{\prime}\right)}\right) \in\left[\left(q_{\lambda}+\frac{m^{\prime}-1}{m^{\prime}}\right) \theta_{\lambda}^{\prime},\left(q_{\lambda}+\frac{m^{\prime}-1}{m^{\prime}}\right) \theta_{\lambda}^{\prime \prime}\right],
$$

and thus

$\operatorname{Re}\left(e^{-\left(q_{\lambda}+\left(\left(m^{\prime}-1\right) / m^{\prime}\right)\right) \theta_{\lambda}^{\prime \prime \prime}} x_{1}^{\ell} t^{q_{\lambda}-\ell+\left(\left(m^{\prime}-1\right) / m^{\prime}\right)}\right) \geq \sin \left(\frac{\pi}{2 m^{\prime}\left(q_{\lambda}+1\right)}\right)\left|x_{1}\right|^{\ell}|t|^{q_{\lambda}-\ell+\left(\left(m^{\prime}-1\right) / m^{\prime}\right)}$.

It follows that

$$
\begin{aligned}
\left|x_{1}^{q_{\lambda}+1}-t^{q_{\lambda}+1}\right| & \geq a^{-1} \sum_{\substack{0 \leq \ell \leq q_{\lambda}+\left(\left(m^{\prime}-1\right) / m^{\prime}\right) \\
\ell \in \mathbf{Z}_{+} / m^{\prime}}}\left|x_{1}^{1 / m^{\prime}}-t^{1 / m^{\prime}}\right|\left|x_{1}\right|^{\ell}|t|^{q_{\lambda}-\ell+\left(\left(m^{\prime}-1\right) / m^{\prime}\right)} \\
& \geq a^{-2}\left|x_{1}^{1 / m^{\prime}}-t^{1 / m^{\prime}}\right|\left(\left|x_{1}\right|^{1 / m^{\prime}}+|t|^{1 / m^{\prime}}\right)^{m^{\prime} q_{\lambda}+m^{\prime}-1}
\end{aligned}
$$

Since we have $q_{\mu} \geq q_{\lambda+1}$, it follows that

$$
\begin{aligned}
\left|x_{1}^{q_{\mu}+1}-t^{q_{\mu}+1}\right| & \leq a\left|x_{1}^{1 / m^{\prime}}-t^{1 / m^{\prime}}\right|\left(\left|x_{1}\right|^{1 / m^{\prime}}+|t|^{1 / m^{\prime}}\right)^{m^{\prime} q_{\mu}+m^{\prime}-1} \\
& \leq a^{-7}\left|x_{1}^{1 / m^{\prime}}-t^{1 / m^{\prime}}\right|\left(\left|x_{1}\right|^{1 / m^{\prime}}+|t|^{1 / m^{\prime}}\right)^{m^{\prime} q_{\lambda}+m^{\prime}-1} \\
& \leq a^{-5}\left|x_{1}^{q_{\lambda}+1}-t^{q_{\lambda}+1}\right|
\end{aligned}
$$

Therefore we have 


$$
\begin{aligned}
\mathrm{I} & \leq-\left(a^{3}\left(q_{\lambda}+1\right)\right)^{-1}\left|x_{1}^{q_{\lambda}+1}-t^{q_{\lambda}+1}\right| \operatorname{Im} \xi_{n}+a\left|x_{1}^{q_{\mu}+1}-t^{q_{\mu}+1}\right| \operatorname{Im} \xi_{n} \\
& \leq-\left(2 a^{3}\left(q_{\lambda}+1\right)\right)^{-1}\left|x_{1}^{q_{\lambda}+1}-t^{q_{\lambda}+1}\right| \operatorname{Im} \xi_{n},
\end{aligned}
$$

and we obtain (17).

We next prove

$$
|\mathrm{II}|,|\mathrm{III}|,|\mathrm{IV}| \leq 2 a^{-7}\left|x_{1}^{q_{\lambda}+1}-t^{q_{\lambda}+1}\right| \operatorname{Im} \xi_{n} .
$$

Let $q_{\lambda}=q_{\mu}$. In this case (20) for II is a direct consequence of (18). Let $q_{\lambda} \neq q_{\mu}$. In this case from (18) and (19) we obtain

$$
\begin{aligned}
|\mathrm{II}| & \leq a^{-7}\left|x_{1}^{q_{\lambda}+1}-t^{q_{\lambda}+1}\right| \operatorname{Im} \xi_{n}+a\left|x_{1}^{q_{\mu}+1}-t^{q_{\mu}+1}\right| \operatorname{Im} \xi_{n} \\
& \leq 2 a^{-7}\left|x_{1}^{q_{\lambda}+1}-t^{q_{\lambda}+1}\right| \operatorname{Im} \xi_{n} .
\end{aligned}
$$

We next prove (20) for III. We have

$$
\begin{aligned}
|\mathrm{III}| & \leq \int_{\delta_{\lambda, \mu}^{ \pm}\left(x_{1}\right) \backslash \delta_{\lambda, \mu}^{ \pm}\left(x_{1}\right)}\left(\left|s^{q_{\lambda}+\left(1 / m^{\prime}\right)} a_{\lambda}^{\prime}\left(s, x^{\prime}, \xi^{\prime}\right)\right|+\left|s^{q_{\mu}+\left(1 / m^{\prime}\right)} a_{\mu}^{\prime}\left(s, x^{\prime}, \xi^{\prime}\right)\right|\right)|d s| \\
& \leq \int_{\gamma_{\lambda, \mu}^{ \pm}\left(x_{1}\right) \backslash \gamma_{\lambda, \mu}^{ \pm}\left(x_{1}\right)} a|s|^{1 /\left(m^{\prime} q_{\lambda}+m^{\prime}\right)} \operatorname{Im} \xi_{n}|d s| \leq 2 a^{-7}\left|x_{1}^{q_{\lambda}+1}-t^{q_{\lambda}+1}\right| \operatorname{Im} \xi_{n},
\end{aligned}
$$

and we obtain (20) for III. The proof for IV is similar. We obtain Lemma 11 from (17) and (20). Q.E.D.

From (ii) of Lemma 10 and Lemma 11 we obtain the following

Corollary. Let $1 \leq \lambda<\mu \leq m,\left(x, \xi^{\prime}\right) \in \Omega_{\lambda}^{\prime}, t \in \delta_{\lambda, \mu}^{ \pm}\left(x_{1}\right)$. Then we have

$$
\begin{aligned}
& \left|\partial_{x^{\prime}}^{\alpha^{\prime}} \partial_{\xi^{\prime}}^{\beta^{\prime}} \exp \left( \pm \operatorname{Re} \varphi_{\lambda, \mu}\left(x, t, \xi^{\prime}\right)\right)\right| \\
& \quad \leq C_{\lambda}^{\left|\alpha^{\prime}+\beta^{\prime}\right| / 5} \alpha^{\prime} ! \beta^{\prime} !\left(\operatorname{Im} \xi_{n}\right)^{-\left|\beta^{\prime}\right|} \exp \left(-a^{-5}\left|t^{q_{\lambda}+1}-x_{1}^{q_{\lambda}+1}\right| \operatorname{Im} \xi_{n}\right) .
\end{aligned}
$$

\section{§6. Diagonalization of the Complete Symbol}

\section{§6.1. Calculation of Some Matrices}

Let

$$
\bar{L}_{i, j}^{\prime \prime}= \begin{cases}\bar{L}_{i, j}^{\prime}, & i=0, \\ 0, & i \neq 0, j=0, \\ \bar{L}_{i, j-1}^{\prime}, & i \neq 0, j \neq 0 .\end{cases}
$$

We have $\sum_{i, j} \bar{L}_{i, j}^{\prime}=\sum_{i, j} \bar{L}_{i, j}^{\prime \prime}$, and these two formal series are essentially the same.

We define $\bar{M}\left(x, \xi^{\prime}\right)\left(=\bar{M}_{0,0}\left(x, \xi^{\prime}\right)\right)$ by 


$$
\bar{M}\left(x, \xi^{\prime}\right)=\left(\begin{array}{ccc}
-\varphi_{1}\left(x, \xi^{\prime}\right) & & 0 \\
& \ddots & \\
0 & & -\varphi_{m}\left(x, \xi^{\prime}\right)
\end{array}\right) .
$$

We study the following equation:

$$
\partial_{x_{1}} V\left(x, \xi^{\prime}\right)+\bar{L}^{\prime \prime}\left(x, \xi^{\prime}\right) \circ V\left(x, \xi^{\prime}\right)-V\left(x, \xi^{\prime}\right) \circ \bar{M}\left(x, \xi^{\prime}\right) \underset{i, j}{=} O .
$$

The essential part is the following

Proposition 1. There exist $m \times m$ matrices $U^{( \pm)}\left(x, \xi^{\prime}\right)=\sum_{0 \leq j \leq i} U_{i, j}^{( \pm)}\left(x, \xi^{\prime}\right)$, and $\bar{M}^{\prime}\left(x, \xi^{\prime}\right)=\sum_{0 \leq j \leq i} \bar{M}_{i, j}^{\prime}\left(x, \xi^{\prime}\right)$ of formal series satisfying the following conditions:

(i) All the components $U_{i, j,(\mu, v)}^{( \pm)}$and $\bar{M}_{i, j,(\mu, v)}^{\prime}$ are holomorphic on $\Omega_{m}^{\prime}$.

(ii) We have

$$
\begin{gathered}
U^{( \pm)} \circ U^{(\mp)} \underset{i, j}{=} I_{m}, \\
\partial_{x_{1}} U^{(+)}+\bar{L}^{\prime \prime} \circ U^{(+)}-U^{(+)} \circ \bar{M}^{\prime} \underset{i, j}{=} O \\
\partial_{x_{1}} U^{(-)}+\bar{M}^{\prime} \circ U^{(-)}-U^{(-)} \circ \bar{L}^{\prime \prime} \underset{i, j}{=} O
\end{gathered}
$$

on $\Omega_{m}^{\prime}$.

(iii) $\bar{M}^{\prime}$ is a diagonal matrix and we have

$$
\begin{gathered}
\mathcal{N}\left(U_{(\mu, v)}^{( \pm)}-\delta_{\mu \nu}, \Omega_{m}^{\prime}, t_{1}, t_{2}\right) \leq 3 m^{3} a^{-1} \\
\mathcal{N}\left(\bar{M}_{(\mu, v)}^{\prime}+\delta_{\mu \nu} \varphi_{\mu}, \Omega_{m}^{\prime}, t_{1}, t_{2}\right) \leq C_{m}\left|x_{1}\right|^{-q(m-1)-1}
\end{gathered}
$$

if $0<t_{1}, t_{2} \ll 1$.

Precisely speaking, $\Omega_{m}^{\prime}$, and thus $U^{( \pm)}$are dependent on $\theta$, and we should have written as $\Omega_{m}^{(\theta)}$ and $U^{( \pm, \theta)}$. But we omit $\theta$ for the moment.

We can prove Proposition 1 inductively for the rows and columns of the matrices. We first find some matrices $\bar{M}^{(\lambda)}=\sum_{0 \leq j \leq i} \bar{M}_{i, j}^{(\lambda)}, U^{(+, \lambda)}=\sum_{0 \leq j \leq i} U_{i, j}^{(+, \lambda)}$, $U^{(-, \lambda)}=\sum_{0 \leq j \leq i} U_{i, j}^{(-, \lambda)}(0 \leq \lambda \leq m)$ such that $\bar{M}_{i, j,(\mu, v)}^{(\lambda)}, U_{i, j,(\mu, v)}^{(+, \lambda)}, U_{i, j,(\mu, v)}^{(-, \lambda)} \in \mathcal{O}\left(\Omega_{\lambda}^{\prime}\right)$, and

$$
\left\{\begin{array}{l}
\bar{M}_{(\mu, v)}^{(\lambda)}=0, \quad \min (\mu, v) \leq \lambda \text { and } \mu \neq v, \\
U_{(\mu, v)}^{(+, \lambda)}=\delta_{\mu v}, \quad \min (\mu, v) \neq \lambda \text { or } \mu=v \\
\partial_{x_{1}} U^{(+, \lambda)}+\bar{M}^{(\lambda-1)} \circ U^{(+, \lambda)}-U^{(+, \lambda)} \circ \bar{M}^{(\lambda)}=O \\
U^{(+, \lambda)} \circ U^{(-, \lambda)}=U^{(-, \lambda)} \circ U^{(+, \lambda)}=I_{m}
\end{array}\right.
$$


(Here we define $\bar{M}^{(\lambda-1)}=\bar{L}^{\prime \prime}$ if $\lambda=0$ ). In other words, we have

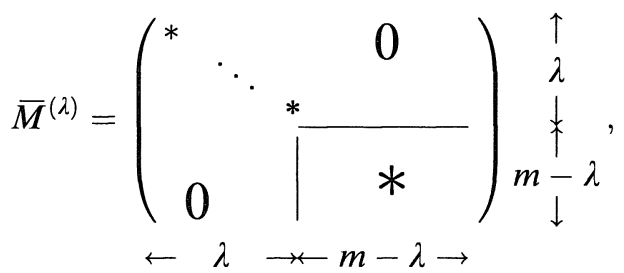

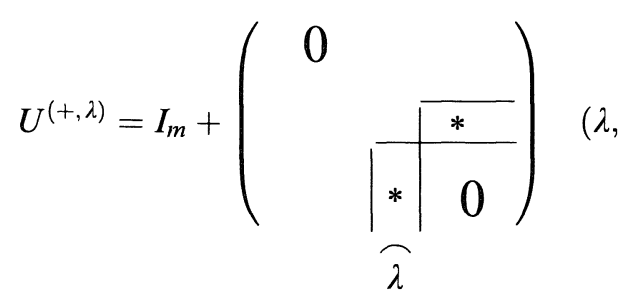

and

$$
D_{1} I_{m}+\bar{M}^{(-1)} \stackrel{U^{(+, 0)}}{\longrightarrow} D_{1} I_{m}+\bar{M}^{(0)} \stackrel{U^{(+, 1)}}{\longrightarrow} \cdots \stackrel{U^{(+, m)}}{\longrightarrow} D_{1} I_{m}+\bar{M}^{(m)}
$$

Proposition 1 is a consequence of the following

Proposition 2. Let $0 \leq \lambda \leq m$. There exists some $\bar{M}_{i, j}^{(\lambda)}, \quad U_{i, j}^{(+, \lambda)} \in$ $\mathcal{O}\left(\Omega_{\lambda}^{\prime}\right)^{m \times m}$ such that $\bar{M}^{(\lambda)}, U^{(+, \lambda)}$ satisfy (21) and we have

$$
\left\{\begin{array}{l}
\mathscr{N}\left(U_{(\mu, v)}^{(+, \lambda)}-\delta_{\mu \nu}, \Omega_{\lambda}^{\prime}, t_{1}, t_{2}\right) \leq a^{-1} \\
\mathscr{N}\left(\bar{M}_{(\mu, v)}^{(\lambda)}+\delta_{\mu \nu} \varphi_{\mu}, \Omega_{\lambda}^{\prime}, t_{1}, t_{2}\right) \leq C_{\lambda}\left|x_{1}\right|^{-q(m-1)-1}
\end{array}\right.
$$

for $0<t_{1}<C_{\lambda}^{-1}, 0<t_{2}<a^{7(m+1-\lambda)}$.

We prove Proposition 2 by induction on $\lambda$. Let us denote $U^{(+, \lambda)}$ by $U^{(\lambda)}$. If $\lambda=0$, we only need to let $U^{(0)}=I_{m}, \bar{M}^{(0)}=\bar{L}^{\prime \prime}$. Let $1 \leq \lambda_{0} \leq m$ and assume that Proposition 2 is true for $0 \leq \lambda \leq \lambda_{0}-1$. Now we prove it for $\lambda=\lambda_{0}$ (Therefore we assume that $U^{(\lambda-1)}$ and $\bar{M}^{(\lambda-1)}$ are already known). We write $U^{\prime(\lambda)}=U^{(\lambda)}-I_{m}$. Then we need to solve

$$
\partial_{x_{1}} U^{\prime(\lambda)}+\bar{M}^{(\lambda-1)}-\bar{M}^{(\lambda)}-\bar{M}^{(\lambda-1)} \circ U^{\prime(\lambda)}-U^{\prime(\lambda)} \circ \bar{M}^{(\lambda)}=O
$$

where $U_{(\mu, v)}^{\prime(\lambda)}=0$ if $\min (\mu, v) \neq \lambda$ or $\mu=v$. Let us calculate the $(\mu, v)$ component of (23). It follows from $(21)$ that the $(\mu, v)$-component in the lefthand side of (23) is equal to the following: 


$$
\begin{cases}\bar{M}_{(\mu, v)}^{(\lambda-1)}-\bar{M}_{(\mu, v)}^{(\lambda)}, & \text { if } \min (\mu, v) \leq \lambda-1, \\ \bar{M}_{(\mu, v)}^{(\lambda-1)}-\bar{M}_{(\mu, v)}^{(\lambda)}+\bar{M}_{(\mu, \lambda)}^{(\lambda-1)} \circ U_{(\lambda, v)}^{\prime(\lambda)}, & \text { if } \min (\mu, v) \geq \lambda+1, \\ \bar{M}_{(\mu, v)}^{(\lambda-1)}-\bar{M}_{(\mu, v)}^{(\lambda)}+\sum_{\kappa \geq \lambda+1} \bar{M}_{(\mu, \kappa)}^{(\lambda-1)} \circ U_{(\kappa, v)}^{(\lambda)}, & \text { if } \min (\mu, v)=\lambda \text { and } \mu=v, \\ \partial_{x_{1}} U_{(\mu, v)}^{(\lambda)}+\bar{M}_{(\mu, v)}^{(\lambda-1)}-\bar{M}_{(\mu, v)}^{(\lambda)}+\sum_{\kappa \geq \lambda}\left(\bar{M}_{(\mu, \kappa)}^{(\lambda-1)} \circ U_{(\kappa, v)}^{\prime(\lambda)}-U_{(\mu, \kappa)}^{(\lambda)} \circ \bar{M}_{(\kappa, v)}^{(\lambda)}\right), \\ & \text { if } \min (\mu, v)=\lambda \text { and } \mu \neq v .\end{cases}
$$

This means that if we obtain $U^{\prime(\lambda)}$, then we must define

(24) $\bar{M}_{(\mu, v)}^{(\lambda)}= \begin{cases}\bar{M}_{(\mu, v)}^{(\lambda-1)}, & \text { if } \min (\mu, v) \leq \lambda-1, \\ \bar{M}_{(\mu, v)}^{(\lambda-1)}+\bar{M}_{(\mu, \lambda)}^{(\lambda-1)} \circ U_{(\lambda, v)}^{\prime(\lambda)}, & \text { if } \min (\mu, v) \geq \lambda+1, \\ \bar{M}_{(\mu, v)}^{(\lambda-1)}+\sum_{\kappa \geq \lambda+1} \bar{M}_{(\mu, \kappa)}^{(\lambda-1)} \circ U_{(\kappa, v)}^{\prime(\lambda)}, & \text { if } \min (\mu, v)=\lambda \text { and } \mu=v, \\ 0, & \text { if } \min (\mu, v)=\lambda \text { and } \mu \neq v .\end{cases}$

On the other hand, we must define $U^{\prime(\lambda)}$ by

$$
\partial_{x_{1}} U_{(\mu, v)}^{\prime(\lambda)}+\bar{M}_{(\mu, v)}^{(\lambda-1)}-\bar{M}_{(\mu, v)}^{(\lambda)}+\sum_{\kappa \geq \lambda}\left(\bar{M}_{(\mu, \kappa)}^{(\lambda-1)} \circ U_{(\kappa, v)}^{\prime(\lambda)}-U_{(\mu, \kappa)}^{\prime(\lambda)} \circ \bar{M}_{(\kappa, v)}^{(\lambda)}\right)=0
$$

for $\min (\mu, v)=\lambda, \mu \neq v$. Substituting (24) into (25), we can eliminate $\bar{M}_{(\mu, v)}^{(\lambda)}$ from (25). We define $A(\mu, v)$ and $B(\mu, v)$ by

$$
A(\mu, v)=\{\lambda\}, \quad B(\mu, v)=\{(l, \kappa) ; l=\lambda, \lambda+1 \leq \kappa \leq m\}
$$

if $\mu>v=\lambda$, and

$$
A(\mu, v)=\{\lambda+1, \ldots, m\}, \quad B(\mu, v)=\{(l, \kappa) ; \lambda+1 \leq \imath \leq m, \kappa=\lambda\}
$$

if $v>\mu=\lambda$. Then we obtain

$$
\begin{aligned}
\partial_{x_{1}} U_{(\mu, \nu)}^{\prime(\lambda)}+\bar{M}_{(\mu, v)}^{(\lambda-1)}+\sum_{\kappa \geq \lambda} \bar{M}_{(\mu, \kappa)}^{(\lambda-1)} \circ U_{(\kappa, v)}^{\prime(\lambda)} \\
\quad-\sum_{\kappa \in A(\mu, v)} U_{(\mu, \kappa)}^{\prime(\lambda)} \circ \bar{M}_{(\kappa, v)}^{(\lambda-1)}-\sum_{(l, \kappa) \in B(\mu, v)} U_{(\mu, l)}^{(\lambda)} \circ \bar{M}_{(l, \kappa)}^{(\lambda-1)} \circ U_{(\kappa, v)}^{\prime(\lambda)}=0
\end{aligned}
$$

for $\min (\mu, v)=\lambda, \mu \neq v$. Therefore we need to solve (26) for $U_{(\mu, v)}^{\prime(\lambda)}$ at first, and define $\bar{M}_{(\mu, v)}^{(\lambda)}$ by (24), after that. We will do this in the next section. 


\section{§6.2. A Study of Ordinary Differential Equations}

We solve (26) by the following successive approximation. Let $\min (\mu, v)$ $=\lambda, \mu \neq v$. We consider

$$
\partial_{x_{1}} U_{i, j,(\mu, v)}^{\prime(\lambda, k)}+\left(\bar{L}_{0,(\mu, \mu)}^{\prime \prime}-\bar{L}_{0,(v, v)}^{\prime \prime}\right) U_{i, j,(\mu, v)}^{\prime(\lambda, k)}=F_{i, j,(\mu, v)}^{(\lambda, k)},
$$

where $F_{i, j,(\mu, v)}^{(\lambda, k)}=\sum_{0 \leq \ell \leq 3} F_{i, j,(\mu, v)}^{(\lambda, k, \ell)}$ and

$$
\begin{aligned}
F_{(\mu, v)}^{(\lambda, k, 0)}= & -\bar{M}_{(\mu, v)}^{(\lambda-1)}, \\
F_{(\mu, v)}^{(\lambda, k, 1)}= & -\sum_{\kappa \geq \lambda}\left(\bar{M}_{0,(\mu, \kappa)}^{(\lambda-1)}-\delta_{\mu \kappa} \bar{L}_{0,(\mu, \mu)}^{\prime \prime}\right) U_{(\kappa, v)}^{(\lambda, k-1)} \\
& +\sum_{\kappa \in A(\mu, v)}\left(\bar{M}_{0,(\kappa, v)}^{(\lambda-1)}-\delta_{\kappa v} \bar{L}_{0,(v, v)}^{\prime \prime}\right) U_{(\mu, \kappa)}^{(\lambda, k-1)}, \\
F_{i, j,(\mu, v)}^{(\lambda, k, 2)}= & -\sum_{(27)} \frac{1}{\alpha^{\prime} !} \partial_{\xi^{\prime}}^{\alpha^{\prime}} \bar{M}_{i^{\prime}, j^{\prime},(\mu, \kappa)}^{(\lambda-1)} \partial_{x^{\prime}}^{\alpha^{\prime}} U_{i^{\prime \prime}, j^{\prime \prime}(\kappa, v)}^{\prime(\lambda, k-1)} \\
& +\sum_{(28)} \frac{1}{\alpha^{\prime} !} \partial_{x^{\prime}}^{\alpha^{\prime}} \bar{M}_{i^{\prime}, j^{\prime},(\kappa, v)}^{(\lambda-1)} \partial_{\xi^{\prime}}^{\alpha^{\prime}} U_{i^{\prime \prime}, j^{\prime \prime}(\mu, \kappa)}^{\prime(\lambda, k-1)}, \\
F_{(\mu, v)}^{(\lambda, k, 3)}= & \sum_{(l, \kappa) \in B(\mu, v)} U_{(\mu, l)}^{\prime(\lambda, k-1)} \circ \bar{M}_{(l, \kappa)}^{(\lambda-1)} \circ U_{(\kappa, v)}^{(\lambda, k-1)} .
\end{aligned}
$$

Here we have defined

$$
\begin{aligned}
& i^{\prime}+i^{\prime \prime}+\left|\alpha^{\prime}\right|=i, \quad i^{\prime \prime} \neq i, \\
& j^{\prime}+j^{\prime \prime}=j, \quad j^{\prime} \leq i^{\prime}, \quad j^{\prime \prime} \leq i^{\prime \prime}, \quad \lambda \leq \kappa \leq m,
\end{aligned}
$$

and

$$
\begin{aligned}
& i^{\prime}+i^{\prime \prime}+\left|\alpha^{\prime}\right|=i, \quad i^{\prime \prime} \neq i, \\
& j^{\prime}+j^{\prime \prime}=j, \quad j^{\prime} \leq i^{\prime}, \quad j^{\prime \prime} \leq i^{\prime \prime}, \quad \kappa \in A(\mu, v)
\end{aligned}
$$

for $i^{\prime}, i^{\prime \prime}, j^{\prime}, j^{\prime \prime}, \kappa \in \mathbf{Z}_{+}$, and $\alpha^{\prime} \in \mathbb{Z}_{+}^{n-1}$ (We have written $U^{\prime(\lambda, k)}=F^{(\lambda, k)}=$ $F^{(\lambda, k, \ell)}=O$ if $\left.k<0\right)$. Let

$$
\delta_{\mu, v}\left(x_{1}\right)=\delta_{\mu, v}^{+}\left(x_{1}\right)
$$

if $\mu<v$, and

$$
\delta_{\mu, v}\left(x_{1}\right)=\delta_{v, \mu}^{-}\left(x_{1}\right), \varphi_{\mu, v}\left(x, \xi^{\prime}\right)=-\varphi_{v, \mu}\left(x, \xi^{\prime}\right)
$$

if $\mu>v$. Note that if we have decided $U_{(\mu, v)}^{\prime(\lambda, k-1)}$, then we can determine $F_{(\mu, v)}^{(\lambda, k, \ell)}$. Therefore we may define $U_{(\mu, v)}^{\prime(\lambda, k)}$ by 


$$
U_{(\mu, v)}^{\prime(\lambda, k)}\left(x, \xi^{\prime}\right)=\int_{\delta_{\mu v}\left(x_{1}\right)} \exp \left(\varphi_{\mu, v}\left(x, s, \xi^{\prime}\right)\right) F_{(\mu, v)}^{(\lambda, k)}\left(s, x^{\prime}, \xi^{\prime}\right) d s .
$$

We want to prove that $\lim _{k \rightarrow \infty} U_{(\mu, v)}^{\prime(\lambda, k)} \in \mathscr{R}^{\theta}$ exists and it satisfies (26). For this purpose, we define $\tilde{U}^{\prime(\lambda, k)}=U^{\prime(\lambda, k)}-U^{(\lambda, k-1)}, \tilde{F}^{(\lambda, k)}=F^{(\lambda, k)}-F^{(\lambda, k-1)}$, and $\tilde{F}^{(\lambda, k, \ell)}=F^{(\lambda, k, \ell)}-F^{(\lambda, k-1, \ell)}$. Therefore we need to consider

$$
\partial_{x_{1}} \tilde{U}_{i, j,(\mu, v)}^{\prime(\lambda, k)}+\left(\bar{L}_{0,(\mu, \mu)}^{\prime \prime}-\bar{L}_{0,(v, v)}^{\prime \prime}\right) \tilde{U}_{i, j,(\mu, v)}^{\prime(\lambda, k)}=\tilde{F}_{i, j,(\mu, v)}^{(\lambda, k)},
$$

where $\tilde{F}_{i, j,(\mu, v)}^{(\lambda, k)}=\sum_{0 \leq \ell \leq 3} \tilde{F}_{i, j,(\mu, v)}^{(\lambda, k, \ell)}$ and

$$
\begin{aligned}
\tilde{F}_{(\mu, v)}^{(\lambda, k, 0)}= & -\delta_{k 0} \bar{M}_{(\mu, v)}^{(\lambda-1)} \\
\tilde{F}_{(\mu, v)}^{(\lambda, k, 1)}= & -\sum_{\kappa \geq \lambda}\left(\bar{M}_{0,(\mu, \kappa)}^{(\lambda-1)}-\delta_{\mu \kappa} \bar{L}_{0,(\mu, \mu)}^{\prime \prime}\right) \tilde{U}_{(\kappa, v)}^{\prime(\lambda, k-1)} \\
& +\sum_{\kappa \in A(\mu, v)}\left(\bar{M}_{0,(\kappa, v)}^{(\lambda-1)}-\delta_{\kappa v} \bar{L}_{0,(v, v)}^{\prime \prime}\right) \tilde{U}_{(\mu, \kappa)}^{(\lambda, k-1)} \\
\tilde{F}_{i, j,(\mu, v)}^{(\lambda, k, 2)}= & -\sum_{(27)} \frac{1}{\alpha^{\prime} !} \partial_{\xi^{\prime}}^{\alpha^{\prime}} \bar{M}_{i^{\prime}, j^{\prime},(\mu, \kappa)}^{(\lambda-1)} \partial_{x^{\prime}}^{\alpha^{\prime}} \tilde{U}_{i^{\prime \prime}, j^{\prime \prime},(\kappa, v)}^{\prime(\lambda, k-1)} \\
& +\sum_{(28)} \frac{1}{\alpha^{\prime} !} \partial_{x^{\prime}}^{\alpha^{\prime}} \bar{M}_{i^{\prime}, j^{\prime},(\kappa, v)}^{(\lambda-1)} \partial_{\xi^{\prime}}^{\alpha^{\prime}} \tilde{U}_{i^{\prime \prime}, j^{\prime \prime},(\mu, \kappa)}^{\prime(\lambda, k-1)}, \\
\tilde{F}_{(\mu, v)}^{(\lambda, k, 3)}= & \sum_{(l, \kappa) \in B(\mu, v)}\left\{U_{(\mu, l)}^{\prime(\lambda, k-1)} \circ \bar{M}_{(l, \kappa)}^{(\lambda-1)} \circ \tilde{U}_{(\kappa, v)}^{\prime(\lambda, k-1)}\right. \\
& \left.+\tilde{U}_{(\mu, l)}^{(\lambda, k-1)} \circ \bar{M}_{(l, \kappa)}^{(\lambda-1)} \circ U_{(\kappa, v)}^{\prime(\lambda, k-2)}\right\} .
\end{aligned}
$$

Let us define

$$
\tilde{U}_{(\mu, v)}^{\prime(\lambda, k)}\left(x, \xi^{\prime}\right)=\int_{\delta_{\mu, 1}\left(x_{1}\right)} \exp \left(\varphi_{\mu, v}\left(x, s, \xi^{\prime}\right)\right) \tilde{F}_{(\mu, v)}^{\prime(\lambda, k)}\left(s, x^{\prime}, \xi^{\prime}\right) d s .
$$

Then we have the following

Lemma 12. If $\min (\mu, v)=\lambda, \mu \neq v, 0<t_{1}<C_{\lambda}^{-1}, 0<t_{2}<a^{7(m+1-\lambda)}$, then we have

$$
\mathcal{N}\left(\tilde{U}_{(\mu, v)}^{\prime(\lambda, k)}, \Omega_{\lambda}^{\prime}, t_{1}, t_{2}\right) \leq 2^{-k-1} a^{-1}
$$

Proof. Let $k_{0} \in \mathbb{Z}_{+}$and assume that Lemma 12 is true if $0 \leq k \leq k_{0}-1$ (We have also assumed that Proposition 2 is true if we replace the number $\lambda$ by $\lambda-1)$. Then we may assume that 
(29)

$$
\mathcal{N}\left(\tilde{F}_{(\mu, v)}^{(\lambda, k, 0)}, \Omega_{\lambda}^{\prime}\right)=\mathcal{N}\left(\delta_{k 0} \bar{M}_{(\mu, v)}^{(\lambda-1)}, \Omega_{\lambda}^{\prime}\right) \leq \delta_{k 0} C_{\lambda-1}\left|x_{1}\right|^{-q(m-1)-1}
$$

and we can prove

$$
\mathscr{N}\left(\tilde{F}_{(\mu, v)}^{(\lambda, k, \ell)}, \Omega_{\lambda}^{\prime}\right) \leq \begin{cases}4 m a^{-1} 2^{-k} C_{\lambda-1}\left|x_{1}\right|^{-q(m-1)-1}, & \ell=1,3 \\ 4 m a^{-7} 2^{-k}\left|x_{1}\right|^{q_{\lambda}} \operatorname{Im} \xi_{n}, & \ell=2 .\end{cases}
$$

Let us prove (30) for the case $\ell=2$ (The other cases are easier). We have

$$
\begin{aligned}
& \mathcal{N}\left(\tilde{F}_{(\mu, v)}^{(\lambda, k, \ell)}, \Omega_{\lambda}^{\prime}\right) \\
& \leq \sum_{(31)} \frac{2(2 n)^{-i^{\prime}-i^{\prime \prime}-\left|\gamma^{\prime}\right|}\left(i^{\prime}+i^{\prime \prime}+\left|\gamma^{\prime}\right|\right) !\left(\alpha^{\prime(1)}+\alpha^{\prime(2)}\right) !\left(\beta^{\prime(1)}+\beta^{\prime(2)}\right) !}{\left(i^{\prime}+i^{\prime \prime}+\left|\alpha^{\prime(1)}+\alpha^{\prime(2)}+\gamma^{\prime}\right|\right) !\left(i^{\prime}+i^{\prime \prime}+\left|\beta^{\prime(1)}+\beta^{\prime(2)}+\gamma^{\prime}\right|\right) ! \alpha^{\prime(1)} ! \alpha^{\prime(2)} ! \beta^{\prime(1)} ! \beta^{\prime(2)} ! \gamma^{\prime} !} \\
& \times\left|\partial_{x^{\prime}}^{\alpha^{\prime(1)}} \partial_{\xi^{\prime}}^{\beta^{\prime(1)}+\gamma^{\prime}} \bar{M}_{i^{\prime}+j^{\prime}, j^{\prime},(\mu, \kappa)}^{(\lambda-1)}\right|\left|\partial_{x^{\prime}}^{\alpha^{\prime(2)}+\gamma^{\prime}} \partial_{\xi^{\prime}}^{\beta^{\prime(2)}} \tilde{U}_{i^{\prime \prime}+j^{\prime \prime}, j^{\prime \prime},(\kappa, v)}^{\prime(\lambda, k-1)}\right|
\end{aligned}
$$

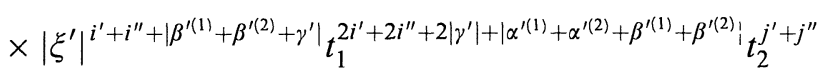

$$
\begin{aligned}
& +\sum_{(32)} \frac{2(2 n)^{-i^{\prime}-i^{\prime \prime}-\left|\gamma^{\prime}\right|}\left(i^{\prime}+i^{\prime \prime}+\left|\gamma^{\prime}\right|\right) !\left(\alpha^{\prime(1)}+\alpha^{\prime(2)}\right) !\left(\beta^{\prime(1)}+\beta^{\prime(2)}\right) !}{\left(i^{\prime}+i^{\prime \prime}+\left|\alpha^{\prime(1)}+\alpha^{\prime(2)}+\gamma^{\prime}\right|\right) !\left(i^{\prime}+i^{\prime \prime}+\left|\beta^{\prime(1)}+\beta^{\prime(2)}+\gamma^{\prime}\right|\right) ! \alpha^{\prime(1)} ! \alpha^{\prime(2)} ! \beta^{\prime(1)} ! \beta^{\prime(2)} ! \gamma^{\prime} !} \\
& \times\left|\partial_{x^{\prime}}^{\alpha^{\prime(1)}+\gamma^{\prime}} \partial_{\xi^{\prime}}^{\beta^{\prime(1)}} \bar{M}_{i^{\prime}+j^{\prime}, j^{\prime},(\kappa, v)}^{(\lambda-1)}\right|\left|\partial_{x^{\prime}}^{\alpha^{\prime(2)}} \partial_{\xi^{\prime}}^{\beta^{\prime(2)}+\gamma^{\prime}} \tilde{U}_{i^{\prime \prime}+j^{\prime \prime}, j^{\prime \prime},(\mu, \kappa)}^{\prime(\lambda, k-1)}\right| \\
& \times\left|\xi^{\prime}\right|^{i^{\prime}+i^{\prime \prime}+\left|\beta^{\prime(1)}+\beta^{\prime(2)}+\gamma^{\prime}\right|} t_{1}^{2 i^{\prime}+2 i^{\prime \prime}+2\left|\gamma^{\prime}\right|+\left|\alpha^{\prime(1)}+\alpha^{\prime(2)}+\beta^{\prime(1)}+\beta^{\prime(2)}\right|} t_{2}^{j^{\prime}+j^{\prime \prime},}
\end{aligned}
$$

where

$$
i^{\prime}+j^{\prime}+\left|\gamma^{\prime}\right| \neq 0, \quad \lambda \leq \kappa \leq m
$$

and

$$
i^{\prime}+j^{\prime}+\left|\gamma^{\prime}\right| \neq 0, \quad \kappa \in A(\mu, v)
$$

for $i^{\prime}, i^{\prime \prime}, j^{\prime}, j^{\prime \prime}, \kappa \in \mathbf{Z}_{+}$, and $\alpha^{\prime(1)}, \alpha^{\prime(2)}, \beta^{\prime(1)}, \beta^{\prime(2)}, \gamma^{\prime} \in \mathbf{Z}_{+}^{n-1}$. Here we have

$$
\begin{aligned}
& \frac{2(2 n)^{-i^{\prime}-i^{\prime \prime}-\left|\gamma^{\prime}\right|}\left(i^{\prime}+i^{\prime \prime}+\left|\gamma^{\prime}\right|\right) !\left(\alpha^{\prime(1)}+\alpha^{\prime(2)}\right) !\left(\beta^{\prime(1)}+\beta^{\prime(2)}\right) !}{\left(i^{\prime}+i^{\prime \prime}+\left|\alpha^{\prime(1)}+\alpha^{\prime(2)}+\gamma^{\prime}\right|\right) !\left(i^{\prime}+i^{\prime \prime}+\left|\beta^{\prime(1)}+\beta^{\prime(2)}+\gamma^{\prime}\right|\right) ! \alpha^{\prime(1)} ! \alpha^{\prime(2)} ! \beta^{\prime(1)} ! \beta^{\prime(2)} ! \gamma^{\prime} !} \\
& \leq \frac{2(2 n)^{-i^{\prime}-i^{\prime \prime}-\left|\gamma^{\prime}\right|} i^{\prime} ! i^{\prime \prime} !\left(i^{\prime}+\left|\gamma^{\prime}\right|\right) !\left(i^{\prime \prime}+\left|\gamma^{\prime}\right|\right) !}{\left(i^{\prime}+\left|\alpha^{\prime(1)}\right|\right) !\left(i^{\prime}+\left|\beta^{\prime(1)}+\gamma^{\prime}\right|\right) !\left(i^{\prime \prime}+\left|\alpha^{\prime(2)}+\gamma^{\prime}\right|\right) !\left(i^{\prime}+\left|\beta^{\prime(2)}\right|\right) !\left(i^{\prime}+i^{\prime \prime}+\left|\gamma^{\prime}\right|\right) ! \gamma^{\prime} !} \\
& \leq \frac{2(2 n)^{-i^{\prime}-i^{\prime \prime}-\left|\gamma^{\prime}\right|} i^{\prime} ! i^{\prime \prime} !\left|\gamma^{\prime}\right| !}{\left(i^{\prime}+\left|\alpha^{\prime(1)}\right|\right) !\left(i^{\prime}+\left|\beta^{\prime(1)}+\gamma^{\prime}\right|\right) !\left(i^{\prime \prime}+\left|\alpha^{\prime(2)}+\gamma^{\prime}\right|\right) !\left(i^{\prime}+\left|\beta^{\prime(2)}\right|\right) ! \gamma^{\prime} !} .
\end{aligned}
$$

Let $\bar{\alpha}^{\prime(1)}=\alpha^{\prime(1)}, \bar{\alpha}^{\prime(2)}=\alpha^{\prime(2)}+\gamma^{\prime}, \bar{\beta}^{\prime(1)}=\beta^{\prime(1)}+\gamma^{\prime}, \bar{\beta}^{\prime(2)}=\beta^{\prime(2)}$. Then it follows that 


$$
\begin{aligned}
\sum_{(31)} \leq & \sum_{(31)^{\prime}} \frac{2(2 n)^{-i^{\prime}-i^{\prime \prime}-\left|\gamma^{\prime}\right|} i^{\prime} ! i^{\prime \prime} !\left|\gamma^{\prime}\right| !}{\left(i^{\prime}+\left|\bar{\alpha}^{\prime(1)}\right|\right) !\left(i^{\prime}+\left|\bar{\beta}^{\prime(1)}\right|\right) !\left(i^{\prime \prime}+\left|\bar{\alpha}^{\prime(2)}\right|\right) !\left(i^{\prime}+\left|\bar{\beta}^{\prime(2)}\right|\right) ! \gamma^{\prime} !} \\
& \times\left|\partial_{x^{\prime}}^{\bar{\alpha}^{\prime(1)}} \partial_{\xi^{\prime}}^{\bar{\beta}^{\prime(1)}} \bar{M}_{i^{\prime}+j^{\prime}, j^{\prime},(\mu, \kappa)}^{(\lambda-1)}\right|\left|\partial_{x^{\prime}}^{\bar{\alpha}^{\prime(2)}} \partial_{\xi^{\prime}}^{\bar{\beta}^{\prime}(2)} \tilde{U}_{i^{\prime \prime}+j^{\prime \prime}, j^{\prime \prime},(\kappa, v)}^{\prime(\lambda, k-1)}\right| \\
& \times\left|\xi^{\prime}\right|^{i^{\prime}+i^{\prime \prime}+\left|\bar{\beta}^{\prime(1)}+\bar{\beta}^{\prime(2)}\right|} t_{1}^{2 i^{\prime}+2 i^{\prime \prime}+\left|\bar{\alpha}^{\prime(1)}+\bar{\alpha}^{\prime(2)}+\bar{\beta}^{\prime(1)}+\bar{\beta}^{\prime(2)}\right| t_{2}^{j^{\prime}+j^{\prime \prime}},}
\end{aligned}
$$

where

$$
i^{\prime}+j^{\prime}+\left|\bar{\alpha}^{(1)}+\bar{\beta}^{\prime(1)}\right| \neq 0, \quad \lambda \leq \kappa \leq m
$$

for $i^{\prime}, i^{\prime \prime}, j^{\prime}, j^{\prime \prime}, \kappa, \bar{\alpha}^{\prime(1)}, \bar{\alpha}^{\prime(2)}, \bar{\beta}^{\prime(1)}, \bar{\beta}^{\prime(2)}, \gamma^{\prime}$. Here we have

$$
\begin{aligned}
\left|\partial_{x^{\prime}}^{\bar{\alpha}^{\prime(1)}} \partial_{\xi^{\prime}}^{\bar{\beta}^{\prime(1)}} \bar{M}_{i^{\prime}+j^{\prime}, j^{\prime},(\mu, \kappa)}^{(\lambda-1)}\right| \leq & \frac{\left(i^{\prime}+\left|\bar{\alpha}^{\prime(1)}\right|\right) !\left(i^{\prime}+\left|\bar{\beta}^{\prime(1)}\right|\right) !}{2(2 n)^{-i^{\prime}} i^{\prime} !} \\
& \times\left|\xi^{\prime}\right|^{-l^{\prime}-\mid \bar{\beta}^{\prime(1)} !} C_{\lambda-1}^{2 i^{\prime}+\left|\bar{\alpha}^{\prime(1)}+\bar{\beta}^{\prime(1)}\right|} a^{-7 j^{\prime}(m+2-\lambda)} a\left|x_{1}\right|^{q_{\lambda}} \operatorname{Im} \xi_{n} .
\end{aligned}
$$

by the assumption of induction (on $\lambda$ ). It follows that

$$
\begin{aligned}
\sum_{(31)} \leq & \sum_{(31)^{\prime}} \frac{(2 n)^{-i^{\prime \prime}-\left|\gamma^{\prime}\right|} i^{\prime \prime} !\left|\gamma^{\prime}\right| !}{\left(i^{\prime}+\left|\bar{\alpha}^{\prime(2)}\right|\right) !\left(i^{\prime}+\left|\bar{\beta}^{\prime(2)}\right|\right) ! \gamma^{\prime} !}\left|\partial_{x^{\prime}}^{\bar{\alpha}^{\prime(2)}} \partial_{\xi^{\prime}}^{\bar{\beta}^{\prime(2)}} \tilde{U}_{i^{\prime \prime}+j^{\prime \prime}, j^{\prime \prime},(\kappa, v)}^{\prime(\lambda, k-1)}\right|\left|\xi^{\prime}\right|^{i^{\prime \prime}+\left|\bar{\beta}^{\prime(2)}\right|} \\
& \times t_{1}^{2 i^{\prime \prime}+\left|\bar{\alpha}^{\prime(2)}+\bar{\beta}^{\prime(2)}\right|} t_{2}^{j^{\prime \prime}}\left(t_{1} C_{\lambda-1}\right)^{2 i^{\prime}+\left|\bar{\alpha}^{\prime(1)}+\bar{\beta}^{\prime(1)}\right|}\left(t_{2} a^{-7(m+2-\lambda)}\right)^{j^{\prime}} a\left|x_{1}\right|^{q_{\lambda}} \operatorname{Im} \xi_{n} .
\end{aligned}
$$

Here we note $0<t_{1} C_{\lambda-1}<C_{\lambda-1} / C_{\lambda} \ll 1$ and $0<t_{2} a^{-7(m+2-\lambda)}<a^{-7}$. By the assumption of induction on $k$, it follows that $\sum_{(31)} \leq 2^{1-k} m a^{-7}\left|x_{1}\right|^{q_{\lambda}} \operatorname{Im} \xi_{n}$. We can similarly prove the same inequality for $\sum_{(32)}$.

From (29) and (30) we obtain

$$
\begin{aligned}
\mathscr{N}\left(\tilde{F}_{(\mu, v)}^{(\lambda, k)}, \Omega_{\lambda}^{\prime}\right) & \leq 2^{1-k} C_{\lambda-1}\left|x_{1}\right|^{-q(m-1)-1}+4 m a^{-7} 2^{-k}\left|x_{1}\right|^{q_{\lambda}} \operatorname{Im} \xi_{n} \\
& \leq 5 m a^{-7} 2^{-k}\left|x_{1}\right|^{q_{\lambda}} \operatorname{Im} \xi_{n} .
\end{aligned}
$$

From Corollary of Lemma 11 it follows that

$$
\mathcal{N}\left(\exp \left( \pm \operatorname{Re} \varphi_{\lambda, \mu}\left(x, t, \xi^{\prime}\right)\right), \Omega_{\lambda}^{\prime}\right) \leq 4 \exp \left(-a^{-5}\left|t^{q_{\lambda}+1}-x_{1}^{q_{\lambda}+1}\right| \operatorname{Im} \xi_{n}\right)
$$

if $t \in \delta_{\lambda, \mu}^{ \pm}$. We have

$$
\begin{aligned}
& \mathcal{N}\left(\tilde{U}_{(\mu, v)}^{\prime(\lambda, k)}\left(x, \xi^{\prime}\right), \Omega_{\lambda}^{\prime}\right) \\
& \quad \leq \int_{\delta_{\mu, v}\left(x_{1}\right)} \mathcal{N}\left(\tilde{U}_{(\mu, v)}^{\prime(\lambda, k)}\left(s, x^{\prime}, \xi^{\prime}\right), \Omega_{\lambda}^{\prime}\right) \mathcal{N}\left(\exp \left(\operatorname{Re} \varphi_{\mu, v}\left(x, s, \xi^{\prime}\right)\right), \Omega_{\lambda}^{\prime}\right)|d s|
\end{aligned}
$$




$$
\begin{aligned}
& \leq \int_{\delta_{\mu, v}\left(x_{1}\right)} 5 m a^{-7} 2^{-k}|s|^{q_{\lambda}} \operatorname{Im} \xi_{n} \cdot 4 \exp \left(-a^{-5}\left|s^{q_{\lambda}+1}-x_{1}^{q_{\lambda}+1}\right| \operatorname{Im} \xi_{n}\right)|d s| \\
& \leq 40 m a^{-2} 2^{-k} \leq 2^{-k-1} a^{-1} .
\end{aligned}
$$

Q.E.D.

Now we can prove Proposition 2. From Lemma 12 we obtain $\mathscr{N}\left(U_{(\mu, v)}^{\prime(\lambda)}, \Omega_{\lambda}^{\prime}, t_{1}, t_{2}\right) \leq a^{-1}$. If we define $\bar{M}^{(\lambda)}$ by $(24)$, we have $(25)$. We can prove (22) directly from this.

We define $U^{(+, \lambda)}=U^{(\lambda)}$, and calculate its inverse series $U^{(-, \lambda)}$ as follows.

Lemma 13. There exists some $U^{(-, \lambda)}=\sum_{0 \leq j \leq i} U_{i, j}^{(-, \lambda)}$, such that

$$
\begin{gathered}
U_{i, j}^{(-, \lambda)} \in\left(\mathcal{O}\left(\Omega_{\lambda}^{\prime}\right)\right)^{m \times m}, \\
U^{( \pm, \lambda)} \circ U^{(\mp, \lambda)}=I_{m} \\
\mathcal{N}\left(U_{(\mu, \nu)}^{(-, \lambda)}-\delta_{\mu \nu}, \Omega_{\lambda}^{\prime}, t_{1}, t_{2}\right) \leq 2 m a^{-1}
\end{gathered}
$$

if $0<t_{1}<C_{\lambda}^{-1}, 0<t_{2}<a^{7(m+1-\ell)}$.

Proof. Let $U^{(-, \lambda)}=I_{m}+\sum_{k \geq 1} \overbrace{U^{\prime(\lambda)} \circ \cdots \circ U^{\prime(\lambda)}}^{k-\text { times }}$. Then it is easy to see that $\mathscr{N}\left(U_{(\mu, v)}^{(-, \lambda)}-\delta_{\mu \nu}, \Omega_{\lambda}^{\prime}\right) \leq \sum_{k \geq 1}\left(m \times \max _{l, \kappa} \mathcal{N}\left(U_{(l, \kappa)}^{(-, \lambda)}, \Omega_{\lambda}^{\prime}\right)\right)^{k} \leq \sum_{k \geq 1}\left(m a^{-1}\right)^{k} \leq 2 m a^{-1}$ and we have $U^{( \pm, \lambda)} \circ U^{(\mp, \lambda)}=I_{m}$. Q.E.D.

If we let $U^{(+)}=U^{(+, 0)} \circ \cdots \circ U^{(+, m)}$ and $U^{(-)}=U^{(-, m)} \circ \cdots \circ U^{(-, 0)}$, we obtain Proposition 1. Our next step is the following

Proposition 3. Let $C^{\prime} \gg C_{m}$, and let $x_{1}^{o}=C^{\prime} \exp \left(\sqrt{-1} \theta_{m}^{\prime \prime} /\left(q_{m}+1\right)\right)$. There exist $V_{i, j,(\mu, v)}^{( \pm)} \in \mathcal{O}\left(\Omega_{m}^{\prime}\left(C^{\prime}\right)\right), 1 \leq j \leq i$, such that $V^{( \pm)}=\sum_{0 \leq j \leq i} V_{i, j}^{( \pm)}$satisfy

$$
\begin{gathered}
V^{( \pm)} \circ V^{(\mp)}=I_{m}, \\
V_{(\mu, v)}^{( \pm)}=0, \quad \mu \neq v,
\end{gathered}
$$

$$
\begin{array}{ll}
\partial_{x_{1}} V^{(+)}+\bar{M}^{(m)} \circ V^{(+)}-V^{(+)} \circ \bar{M} \underset{i, j}{=} O, & V^{(+)}\left(x_{1}^{o}, x^{\prime}, \xi^{\prime}\right) \underset{i, j}{=} I_{m}, \\
\partial_{x_{1}} V^{(-)}+\bar{M} \circ V^{(-)}-V^{(+)} \circ \bar{M}^{(m)} \underset{i, j}{=} O, & V^{(+)}\left(x_{1}^{o}, x^{\prime}, \xi^{\prime}\right) \underset{i, j}{=} I_{m}
\end{array}
$$




$$
\begin{aligned}
& \left|\partial_{x^{\prime}}^{\alpha^{\prime}} \partial_{\xi^{\prime}}^{\beta^{\prime}} V_{i+j, j,(\mu, v)}^{( \pm)}\right| \\
& \leq \sum_{\ell \leq i} \frac{\left(i+\left|\alpha^{\prime}+\beta^{\prime}\right|\right) !}{\ell !} C^{i-(\ell / 4)+\left(\left(\left|\alpha^{\prime}+\beta^{\prime}\right|\right) / 2\right)+1} a^{-6 J} \\
& \quad \times\left|x_{1}-x_{1}^{o}\right|^{\ell}\left(\operatorname{Im} \xi_{n}\right)^{-i+\ell-\left|\beta^{\prime}\right|} \exp \left(C^{\prime}\left|x_{1}-x_{1}^{o}\right|\left(\operatorname{Im} \xi_{n}\right)^{(m q+1) /(m q+m)}\right)
\end{aligned}
$$

on $\Omega_{m}^{\prime}\left(C^{\prime}\right)$.

Proof. Let $\quad \bar{M}_{i^{\prime}, j^{\prime}}=\delta_{i^{\prime} 0} \delta_{j^{\prime} 0} \bar{M} \quad$ and $\quad \bar{M}_{0,0,(\mu, v)}^{\prime}=\bar{M}_{0,0,(\mu, v)}-\bar{M}_{0,0,(\mu, v)}^{(m)}$. Since all the matrices in Proposition 3 are diagonal matrices, we can easily calculate $V_{i, j,(\mu, \mu)}^{(+)}$by solving the following equation:

$$
\begin{aligned}
& \partial_{x_{1}} V_{i, j,(\mu, \mu)}^{(+)}+\bar{M}_{0,0,(\mu, \mu)}^{\prime} V_{i, j,(\mu, \mu)}^{(+)} \\
& \quad=-\sum_{\substack{i^{\prime}+i^{\prime \prime}+\left|\alpha^{\prime}\right|=i \\
i^{\prime \prime} \neq i \\
j^{\prime}+j^{\prime \prime}=j}} \frac{1}{\alpha^{\prime}}\left(\partial_{\xi^{\prime}}^{\alpha^{\prime}} \bar{M}_{i^{\prime}, j^{\prime},(\mu, \mu)}^{(m)} \partial_{x^{\prime}}^{\alpha^{\prime}} V_{i^{\prime \prime}, j^{\prime \prime},(\mu, \mu)}^{(+)}-\partial_{x^{\prime}}^{\alpha^{\prime}} \bar{M}_{i^{\prime}, j^{\prime},(\mu, \mu)} \partial_{\xi^{\prime}}^{\alpha^{\prime}} V_{i^{\prime \prime}, j^{\prime \prime},(\mu, \mu)}^{(+)}\right) .
\end{aligned}
$$

We solve this equation by successive approximation. Let

$$
\left\{\begin{array}{l}
\partial_{x_{1}} V_{i, j,(\mu, \mu)}^{(+, k)}+\bar{M}_{0,0,(\mu, \mu)}^{\prime(m)} V_{i, j,(\mu, \mu)}^{(+, k)}=G_{i, j,(\mu, \mu)}^{(+, k)} \\
V_{i, j,(\mu, \mu)}^{(+, k)}\left(x_{1}^{0}, x^{\prime}, \xi^{\prime}\right)=\delta_{i 0} \delta_{j 0} \delta_{k 0}
\end{array}\right.
$$

where

$$
G_{i, j,(\mu, \mu)}^{(+, k)}=-\sum_{\substack{i^{\prime}+i^{\prime \prime}+\left|\alpha^{\prime}\right|=i \\ i^{\prime \prime \neq i} \\ j^{\prime}+j^{\prime \prime}=j}} \frac{1}{\alpha^{\prime}}\left(\partial_{\xi^{\prime}}^{\alpha^{\prime}} \bar{M}_{i^{\prime}, j^{\prime},(\mu, \mu)}^{(m)} \partial_{x^{\prime}}^{\alpha^{\prime}} V_{i^{\prime \prime}, j^{\prime \prime},(\mu, \mu)}^{(+, k-1)}-\partial_{x^{\prime}}^{\alpha^{\prime}} \bar{M}_{i^{\prime}, j^{\prime},(\mu, \mu)} \partial_{\xi^{\prime}}^{\alpha^{\prime}} V_{i^{\prime \prime}, j^{\prime \prime},(\mu, \mu)}^{(+, k-1)}\right)
$$

for $k \geq 0\left(\right.$ Here $\left.V^{(+,-1)}=O\right)$. Then by a direct calculation we can prove that

$$
\begin{aligned}
\left|\partial_{x^{\prime}}^{\alpha^{\prime}} \partial_{\xi^{\prime}}^{\beta^{\prime}} V_{i+j, j,(\mu, v)}^{(+, k)}\right| \leq & 2^{-k-1} \sum_{\ell \leq i} \frac{\left(i+\left|\alpha^{\prime}+\beta^{\prime}\right|\right) !}{\ell !} C^{\prime i-(\ell / 4)+\left(\left|\alpha^{\prime}+\beta^{\prime}\right| / 2\right)+1} a^{-6 j} \\
& \times\left|x_{1}-x_{1}^{o}\right|^{\ell}\left(\operatorname{Im} \xi_{n}\right)^{-i+\ell-\left|\beta^{\prime}\right|} \exp \left(C^{\prime}\left|x_{1}-x_{1}^{o}\right|\left(\operatorname{Im} \xi_{n}\right)^{(m q+1) /(m q+m)}\right)
\end{aligned}
$$

by induction on $k$. This means that $V^{(+)}=V^{(+, k)}$ satisfies (33) and (35). We can similarly construct $V^{(-)}$satisfying (34) and (35). It follows that

$$
\begin{aligned}
\partial_{x_{1}}\left(V^{(+)} \circ V^{(-)}\right)+ & \bar{M}^{(m)} \circ\left(V^{(+)} \circ V^{(-)}\right)-\left(V^{(+)} \circ V^{(-)}\right) \circ \bar{M}^{(m)}=0, \\
& \left(V^{(+)} \circ V^{(-)}\right)\left(x_{1}^{o}, x^{\prime}, \xi^{\prime}\right)=I_{m},
\end{aligned}
$$

whose unique solution is $V^{(+)} \circ V^{(-)}=I_{m}$. We can similarly prove $V^{(-)} \circ$ $V^{(+)}=I_{m}$. Q.E.D. 


\section{§7. The Last Part of the Proof of Theorem 2}

Here we rewrite the results we have already proved. Let $C \gg C^{\prime}\left(\gg C_{m}\right)$, $\theta \in[0,2 \pi], k \in \mathbf{Z}_{+}$. We define

$$
\begin{gathered}
\Xi(C)=\left\{\left(x, \xi^{\prime}\right) \in \mathbf{C}^{n} \times \mathbf{C}^{n-1} ; C|x|<1, C\left|\xi^{\prime \prime \prime}\right|<\operatorname{Im} \xi_{n},\right. \\
\left.C\left|\operatorname{Re} \xi_{n}\right|<\operatorname{Im} \xi_{n}, C^{4 m(q+1)}<\operatorname{Im} \xi_{n}\right\} \\
\Xi^{\prime}(C, \theta)=\left\{\left(x, \xi^{\prime}\right) \in \Xi(C) ; C\left(\operatorname{Im} \xi_{n}\right)^{-1 /(m q+m)}<\left|x_{1}\right|, C\left|\arg x_{1}-\theta\right|<1\right\}, \\
\Xi^{\prime \prime}(C)=\left\{\left(x, \xi^{\prime}\right) \in \Xi(C) ;\left|x_{1}\right|<C^{-1 / 2}\left(\operatorname{Im} \xi_{n}\right)^{-1 /(2 m q+2 m)}\right\}, \\
\bar{\Xi}^{\prime \prime}(C)=\left\{\left(x, \xi^{\prime}\right) \in \Xi^{\prime \prime}(C) ; x_{1} \neq 0,\left|\arg x_{1}\right|<2 \pi\right\}, \\
\Xi(C, \theta)=\Xi^{\prime}(C, \theta) \cup \Xi^{\prime \prime}(C), \\
\bar{\Xi}(C, \theta)=\Xi^{\prime}(C, \theta) \cup \bar{\Xi}^{\prime \prime}(C), \\
\Xi_{k}(C)=\left\{\left(x, \xi^{\prime}\right) \in \Xi(C) ; C^{4 m(q+1)}(k+1)<\operatorname{Im} \xi_{n}\right\} \\
\Xi_{k}^{\prime}(C, \theta)=\Xi^{\prime}(C, \theta) \cap \Xi_{k}(C), \\
\bar{\Xi}_{k}^{\prime \prime}(C)=\bar{\Xi}^{\prime \prime}(C) \cap \Xi_{k}(C) .
\end{gathered}
$$

Let $W^{(+, \theta)}=\Delta^{+} \circ U^{(+)} \circ V^{(+)}$, and $W^{(-, \theta)}=V^{(-)} \circ U^{(-)} \circ \Delta^{-}$on $\Xi^{\prime}(C, \theta)\left(\subset \Omega_{m}^{\prime}\right)$. Precisely speaking, $U^{( \pm)}, V^{( \pm)}$, and $\Omega_{m}^{\prime}$ are dependent on $\theta$, and we should have written as $U^{( \pm, \theta)}, V^{( \pm, \theta)}$ and $\Omega_{m}^{\prime(\theta)}$, respectively. To the contrary, $\Delta^{( \pm)}$ are independent of $\theta$. Let $\sigma_{+}(\mu, v)=\mu-1$, and $\sigma_{-}(\mu, v)=1-v$. Then we have

$$
\left\{\begin{array}{l}
\partial_{x_{1}} W^{(+, \theta)}+\bar{L} \circ W^{(+, \theta)}-W^{(+, \theta)} \circ \bar{M}=O \\
\partial_{x_{1}} W^{(-, \theta)}+\bar{M} \circ W^{(-, \theta)}-W^{(-, \theta)} \circ \bar{L}=O
\end{array}\right.
$$

and

$$
\begin{aligned}
\left|\partial_{x^{\prime}}^{\alpha^{\prime}} \partial_{\xi^{\prime}}^{\beta^{\prime}} W_{i,(\mu, v)}^{( \pm, \theta)}\right| \leq & \sum_{j \leq i} C^{i-j+\left|\alpha^{\prime}+\beta^{\prime}\right|+1} a^{-5 j}(i-j) ! \alpha^{\prime} ! \beta^{\prime} !\left|x_{1}\right|^{-q(m-1)} \\
& \times\left(\operatorname{Im} \xi_{n}\right)^{-i+j-\left|\beta^{\prime}\right|+\sigma_{ \pm}(\mu, v)} \exp \left(C\left(\operatorname{Im} \xi_{n}\right)^{(m q+1) /(m q+m)}\right)
\end{aligned}
$$

on $\Xi^{\prime}(C, \theta)$. Finally we need to calculate $W^{( \pm, \theta)}$ on $\Xi^{\prime \prime}(C)$. This means that we have already calculated $W^{( \pm, \theta)}$ on $\left\{\left|\xi_{n}\right| \gg 1 /\left|x_{1}\right|\right\}$, and we need to extend them to the remaining domain. This is very easy. Let $x_{1}^{(k)}=$ $C^{-11 / 4}(k+2)^{-1 /(2 m q+2 m)} e^{\sqrt{-1} \theta}$. We define the distance $\rho^{(k)}\left(x_{1}\right)$ from $x_{1}^{(k)}$ to $x_{1}$ by $\rho^{(k)}\left(x_{1}\right)=\left.|| x_{1}\right|^{1 / m}-\left.\left|x_{1}^{(k)}\right|^{1 / m}\left|+\frac{1}{m}\right| x_{1}\right|^{1 / m}\left|\arg x_{1}-\theta\right|$. Then we have the following 
Lemma 14. If $\left(x, \xi^{\prime}\right) \in \Xi_{k}^{\prime}(C, \theta) \backslash \Xi_{k+2}^{\prime}(C, \theta)$, we have $\left(x_{1}^{(k)}, x^{\prime}, \xi^{\prime}\right) \in$ $\Xi^{\prime}(C, \theta) \cap\left(\bar{\Xi}_{k}^{\prime \prime}(C) \backslash \bar{\Xi}_{k+2}^{\prime \prime}(C)\right)$.

Proof. It is easy to see that $C\left(\operatorname{Im} \xi_{n}\right)^{-1 /(m q+m)}<\left|x_{1}^{(k)}\right|<$ $C^{-1 / 2}\left(\operatorname{Im} \xi_{n}\right)^{-1 /(2 m q+2 m)}$, and the lemma follows immediately. Q.E.D.

Lemma 15. We have

$$
\begin{aligned}
& \left|\partial_{x^{\prime}}^{\alpha^{\prime}} \partial_{\xi^{\prime}}^{\beta^{\prime}} W_{i,(\mu, v)}^{( \pm, \theta)}\right| \\
& \quad \leq \sum_{\substack{0 \leq j \leq i \\
0 \leq \ell}} \frac{\left(i-j+\left|\alpha^{\prime}+\beta^{\prime}\right|\right) !}{\ell !} C^{2 i-2 j+\left|\alpha^{\prime}+\beta^{\prime}\right|+\ell+1} a^{-5 j} \\
& \quad \times\left(\rho^{(k)}\left(x_{1}\right)\right)^{\ell}\left(\operatorname{Im} \xi_{n}\right)^{-i+j+\ell-\left\{\beta^{\prime} \mid+\sigma_{ \pm}(\mu, v)+1\right.} \exp \left(C\left(\operatorname{Im} \xi_{n}\right)^{(m q+1) /(m q+m)}\right)
\end{aligned}
$$

on $\bar{\Xi}_{k}^{\prime \prime}(C) \backslash \bar{\Xi}_{k+2}^{\prime \prime}(C)$.

Proof. As we have already seen, $W^{(+, \theta)}$ satisfies (36) on $\Xi^{\prime}(C, \theta)$. Of course we can uniquely extend this solution to $\bar{\Xi}_{k}^{\prime \prime}(C) \backslash \bar{\Xi}_{k+2}^{\prime \prime}(C)$. To be precise, let us consider the following successive approximation:

$$
W^{(+, \theta, \ell)}\left(x, \xi^{\prime}\right)=\delta_{\ell 0} W^{(+, \theta)}\left(x_{1}^{(k)}, x^{\prime}, \xi^{\prime}\right)-\int_{x_{1}^{(k)}}^{x_{1}} H^{(\ell)}\left(s, x^{\prime}, \xi^{\prime}\right) d s
$$

where $H^{(\ell)}=\bar{L} \circ W^{(+, \theta, \ell-1)}-W^{\prime(+, \theta, \ell-1)} \circ \bar{M}$ for $\ell \in \mathbb{Z}_{+}\left(W^{(+, \theta,-1)}=O\right) . \quad$ By induction on $\ell$, let us prove that

$$
\begin{aligned}
\left|\partial_{x^{\prime}}^{\alpha^{\prime}} \partial_{\xi^{\prime}}^{\beta^{\prime}} W_{i,(\mu, v)}^{\prime(+, \theta, \ell)}\right| & \\
\leq & \sum_{0 \leq j \leq i} \frac{\left(i-j+\left|\alpha^{\prime}+\beta^{\prime}\right|\right) !}{\ell !} C^{2 i-2 j+\left|\alpha^{\prime}+\beta^{\prime}\right|+\ell+1} a^{-5 j} \\
& \times\left(\rho^{(k)}\left(x_{1}\right)\right)^{\ell}\left(\operatorname{Im} \xi_{n}\right)^{-i+j+\ell-\left|\beta^{\prime}\right|+\sigma_{+}(\mu, v)+1} \exp \left(C\left(\operatorname{Im} \xi_{n}\right)^{(m q+1) /(m q+m)}\right)
\end{aligned}
$$

on $\bar{\Xi}_{k}^{\prime \prime}(C) \backslash \bar{\Xi}_{k+2}^{\prime \prime}(C)$. Let $\ell=0$. Since $W^{(+, \theta, 0)}\left(x, \xi^{\prime}\right)=W^{(+, \theta)}\left(x_{1}^{(k)}, x^{\prime}, \xi^{\prime}\right)$ and $\left(x_{1}^{(k)}, x^{\prime}, \xi^{\prime}\right) \in \Xi^{\prime}(C, \theta)$, (39) follows from (37). Assume that $\ell_{0} \geq 1$ and that (39) is true if $0 \leq \ell \leq \ell_{0}-1$. Let us consider the case $\ell=\ell_{0}$. Then we obtain

$$
\begin{aligned}
\left|\partial_{x^{\prime}}^{\alpha^{\prime}} \partial_{\xi^{\prime}}^{\beta^{\prime}} H_{i,(\mu, v)}^{(\ell)}\right| & \\
\leq & \sum_{0 \leq j \leq i} \frac{\left(i-j+\left|\alpha^{\prime}+\beta^{\prime}\right|\right) !}{(\ell-1) !} C^{2 i-2 j+\left|\alpha^{\prime}+\beta^{\prime}\right|+\ell} a^{-5 j} \\
& \times\left(\rho^{(k)}\left(x_{1}\right)\right)^{\ell-1}\left(\operatorname{Im} \xi_{n}\right)^{-i+j+\ell-\left|\beta^{\prime}\right|+\sigma_{+}(\mu, v)+1} \exp \left(C\left(\operatorname{Im} \xi_{n}\right)^{(m q+1) /(m q+m)}\right)
\end{aligned}
$$

on $\bar{\Xi}_{k}^{\prime \prime}(C) \backslash \bar{\Xi}_{k+2}^{\prime \prime}(C)$, and we obtain (39). (38) follows from (39), and we can similarly estimate $W^{\prime(-, \theta)}$. Q.E.D. 
Now we can conclude the following

Proposition 4. We have $W_{i,(\mu, v)}^{( \pm, \theta, \ell)} \in \mathcal{O}(\Xi(C, \theta))$ and

$$
\begin{aligned}
\left|\partial_{x^{\prime}}^{\alpha^{\prime}} \partial_{\xi^{\prime}}^{\beta^{\prime}} W_{i,(\mu, v)}^{( \pm, \theta)}\right| \leq & \sum_{0 \leq j \leq i}\left(i-j+\left|\alpha^{\prime}+\beta^{\prime}\right|\right) ! C^{2 i-2 j+\left|\alpha^{\prime}+\beta^{\prime}\right|+1} a^{-5 j} \\
& \times\left(\operatorname{Im} \xi_{n}\right)^{-i+j-\left|\beta^{\prime}\right|+m} \exp \left(C\left(\operatorname{Im} \xi_{n}\right)^{1-1 /(2 m q+2 m)}\right)
\end{aligned}
$$

on $\Xi(C, \theta)$.

Proof. Let $\left(x, \xi^{\prime}\right) \in \Xi(C, \theta)$. If $\left(x, \xi^{\prime}\right) \in \Xi^{\prime}(C, \theta)$, then (40) is already known. Therefore we may assume that $\left(x, \xi^{\prime}\right) \in \Xi^{\prime \prime}(C)$, but it is sufficient to prove (40) for the case $x_{1} \neq 0$. Then we can choose some $k \in \mathbf{Z}_{+}$for which we have $\left(x, \xi^{\prime}\right) \in \bar{\Xi}_{k}^{\prime \prime}(C) \backslash \bar{\Xi}_{k+2}^{\prime \prime}(C)$. From Lemma 15 it follows that

$$
\begin{aligned}
\left|\partial_{x^{\prime}}^{\alpha^{\prime}} \partial_{\xi^{\prime}}^{\beta^{\prime}} W_{i,(\mu, v)}^{( \pm, \theta)}\right| & \\
\leq & \sum_{0 \leq j \leq i}\left(i-j+\left|\alpha^{\prime}+\beta^{\prime}\right|\right) ! C^{2 i-2 j+\left|\alpha^{\prime}+\beta^{\prime}\right|+1} a^{-5 j} \\
& \quad \times\left(\operatorname{Im} \xi_{n}\right)^{-i+j-\left|\beta^{\prime}\right|+\sigma_{ \pm}(\mu, v)+1} \exp \left(C\left(\operatorname{Im} \xi_{n}\right)^{(m q+1) /(m q+m)}+C \rho^{(k)}\left(x_{1}\right) \operatorname{Im} \xi_{n}\right) .
\end{aligned}
$$

Since we have $\rho^{(k)}\left(x_{1}\right) \leq 10\left|x_{1}\right|^{1 / m} \leq 10 C^{-1 / 2 m}\left(\operatorname{Im} \xi_{n}\right)^{-1 /(2 m q+2 m)}$ on $\Xi^{\prime \prime}(C)$ and $\sigma_{ \pm}(\mu, v)+1 \leq m$, we obtain Proposition 4. Q.E.D.

Corollary. $W^{( \pm, \theta)} \in\left(\mathscr{R}^{\theta}\right)^{m \times m}$.

Now we can prove Theorem 2. Let $\tilde{E}^{(+, \theta)}\left(x, \xi^{\prime}\right)=W^{+, \theta}\left(x, \xi^{\prime}\right) \circ$ $X^{(+)}\left(x, \xi^{\prime}\right) \circ W^{-, \theta}\left(0, x^{\prime}, \xi^{\prime}\right) \quad$ and $\quad \tilde{E}^{(-, \theta)}\left(x, \xi^{\prime}\right)=W^{+, \theta}\left(0, x^{\prime}, \xi^{\prime}\right) \circ X^{(-)}\left(x, \xi^{\prime}\right) \circ$ $W^{-, \theta}\left(x, \xi^{\prime}\right)$. Then $\tilde{E}^{( \pm, \theta)}$ satisfy $(15)$ on $\Xi(C, \theta)$. Since such a solution is unique, it follows that $E^{( \pm)}=\tilde{E}^{( \pm, \theta)}$ on $\Xi(C, \theta)$.

The above discussion is valid for each fixed $\theta \in[0,2 \pi]$. To consider various values of $\theta$ simultaneously, we must denote the large number by $C_{\theta}$ instead of $C$, because it may depend on $\theta$. Let us consider on the whole $\Xi\left(C^{\prime}\right), C^{\prime} \gg 1$. There is a finite set $\left\{\theta_{1}, \ldots, \theta_{s}\right\} \in[0,2 \pi]$, such that $[0,2 \pi] \subset$ $\bigcup_{1 \leq j \leq s}\left(\theta_{j}-1 / C_{\theta_{j}}, \theta_{j}+1 / C_{\theta_{j}}\right)$. If we choose $C^{\prime}=\max \left(C_{\theta_{1}}, \ldots, C_{\theta_{s}}\right)$, then we have $\Xi\left(C^{\prime}\right) \subset \bigcup_{1 \leq j \leq s} \Xi\left(C_{\theta_{j}}, \theta_{j}\right)$. It follows that $E^{( \pm)} \in \mathscr{T}^{m \times m}$. We have trivially $X^{( \pm)} \in \mathscr{T}_{\left(m^{\prime}\right)}^{m \times m}$, and it follows that $F^{( \pm, \theta)} \in \mathscr{T}_{\left(m^{\prime}\right)}^{m \times m}$.

\section{References}

[1] Amano, K. and Nakamura, G., Branching of singularities for degenerate hyperbolic operators, Publ. RIMS, Kyoto Univ., 20 (1984), 225-275.

[2] Aoki, T., Symbols and formal symbols of pseudodifferential operators, Adv. Stud. Pure Math., 4 (1984), 181-208. 
[ 3 ] Boutet de Monvel, L. and Krée, P., Pseudo-differential operators and Gevrey classes, Ann. Inst. Fourier, 17 (1967) 295-323.

[4] Boutet de Monvel, L., Hypoelliptic operators with double characteristics and related pseudodifferential operators, Comm. Pure Appl. Math., 27 (1974), 585-639.

[ 5 ] Kashiwara, M. and Kawai, T., Microhyperbolic pseudodifferential operators I, J. Math. Soc. Japan, 27 (1975), 359-404.

[6] Kataoka, K., Micro-local theory of boundary value problems I, J. Fac. Sci. Univ. Tokyo, 27 (1980), 355-399.

[7] - Structure of solutions of differential equations, Proceedings of a symposium held at Katata/Kyoto, World Sci. Publishing, River Edge, NJ, 1997.

[ 8 ] Nakane, S., Propagation of singularities and uniqueness in the Cauchy problem at a class of doubly characteristic points, Comm. Partial Differential Equations, 6 (1981), 917-927.

[ 9 ] Sato, M., Kawai, T. and Kashiwara, M., Microfunctions and pseudo-differential equations, Lecture Notes in Math., 287 (1973), 265-529, Springer.

[10] Takasaki, K., Singular Cauchy problems for a class of weakly hyperbolic differential operators, Comm. Partial Differential Equations, 7 (1982), 1151-1188.

[11] Uchikoshi, K., Symbol theory of microlocal operators, Publ. RIMS, Kyoto Univ., 24 (1988), 547-584.

[12] — Stokes operators for microhyperbolic equations, J. Math. Sci., Univ. of Tokyo, 5 (1998), 507-545.

[13] Yamane, H., Branching of singularities for some second or third order microhyperbolic operators, J. Math. Sci., Univ. of Tokyo, 2 (1995), 671-749. 